\title{
VARIABLE HARDY SPACES ASSOCIATED WITH OPERATORS SATISFYING DAVIES-GAFFNEY ESTIMATES ON METRIC MEASURE SPACES OF HOMOGENEOUS TYPE
}

\author{
Dachun Yang and Junqiang Zhang* \\ Beijing Normal University, School of Mathematical Sciences \\ Laboratory of Mathematics and Complex Systems, Ministry of Education \\ Beijing 100875, P. R. China; dcyang@bnu.edu.cn \\ Beijing Normal University, School of Mathematical Sciences \\ Laboratory of Mathematics and Complex Systems, Ministry of Education \\ Beijing 100875, P. R. China; zhangjunqiang@mail.bnu.edu.cn
}

\begin{abstract}
Let $(\mathcal{X}, d, \mu)$ be a metric measure space of homogeneous type and $p(\cdot): \mathcal{X} \rightarrow(0,1]$ a variable exponent function satisfying the globally log-Hölder continuous condition. Assume that $L$ is a one-to-one operator of type $\omega$ on $L^{2}(\mathcal{X})$, with $\omega \in[0, \pi / 2)$, which has a bounded holomorphic functional calculus, and whose heat kernel satisfies the Davies-Gaffney estimates. In this article, the authors introduce the variable Hardy space $H_{L}^{p(\cdot)}(\mathcal{X})$ associated with $L$. Then the authors establish the molecular characterization of $H_{L}^{p(\cdot)}(\mathcal{X})$ via the atomic decomposition of variable tent spaces and show that the dual space of $H_{L}^{p(\cdot)}(\mathcal{X})$ is the BMO-type space $\mathrm{BMO}_{p(\cdot), L^{*}}(\mathcal{X})$, where $L^{*}$ denotes the adjoint operator of $L$ on $L^{2}(\mathcal{X})$. In particular, if $L$ is a non-negative self-adjoint operator whose heat kernel has a Gaussian upper bound, the authors then obtain the non-tangential and the radial maximal function characterizations of $H_{L}^{p(\cdot)}(\mathcal{X})$ via establishing its atomic characterization.
\end{abstract}

\section{Introduction}

Let $p(\cdot): \mathbf{R}^{n} \rightarrow(0, \infty)$ be a measurable function. The variable Lebesgue space $L^{p(\cdot)}\left(\mathbf{R}^{n}\right)$ is defined to be the space of all measurable functions $f$ on $\mathbf{R}^{n}$ such that, for some $\lambda \in(0, \infty)$,

$$
\int_{\mathbf{R}^{n}}\left[\frac{|f(x)|}{\lambda}\right]^{p(x)} d x<\infty .
$$

In particular, if $p(\cdot) \equiv p$ is a positive constant, then $L^{p(\cdot)}\left(\mathbf{R}^{n}\right)$ is just the classical Lebesgue space $L^{p}\left(\mathbf{R}^{n}\right)$. The study of variable Lebesgue spaces originated from Orlicz [49] in 1931. Then it was further developed by Nakano [47, 48] and Kováčik and Rákosník [42]. Nowadays the interest in variable function spaces has increased steadily and these variable function spaces have been widely used in harmonic analysis as well as partial differential equations; see, for example, [1, 16, 17, 18, 22, 23, 33, 52]. The study of variable Lebesgue spaces on a (quasi-)metric measure space $\mathcal{X}$ seemingly appeared initially in [34], where, under the assumption that $\mathcal{X}$ is bounded with a

https://doi.org/10.5186/aasfm.2018.4304

2010 Mathematics Subject Classification: Primary 42B30; Secondary 42B35, 42B25, 47A60, 30L99.

Key words: Metric measure space of homogeneous type, variable Hardy space, Davies-Gaffney estimate, non-negative self-adjoint operator, square function, maximal function, molecule, atom.

Dachun Yang is supported by the National Natural Science Foundation of China (Grant Nos. 11571039,11671185 and 11361020).

*Corresponding author. 
doubling measure and $p(\cdot)$ is locally log-Hölder continuous, Harjulehto et al. studied the boundedness of the Hardy-Littlewood maximal function $M$ on $L^{p(\cdot)}(\mathcal{X})$. Then there has been a lot of attention paid to the study of the boundedness of operators on variable Lebesgue spaces over metric measure spaces. More precisely, Gorosito et al. [29] investigated the boundedness of the fractional integral operators on weighted variable Lebesgue spaces with non-doubling measures and, in [41], Kokilashvili and Samko established the boundedness of the Hardy-Littlewood maximal operator on variable Lebesgue spaces over metric measure spaces. Moreover, in [32], Hajibayov and Samko considered the generalized potential operators in variable Lebesgue spaces on bounded quasi-metric measure spaces equipped with doubling measures satisfying the so-called Ahlfors $N$-regular condition and, recently, Adamowicz et al. [2] studied the Hardy-Littlewood maximal operator $M$ on $L^{p(\cdot)}(\mathcal{X})$ when $\mathcal{X}$ is an unbounded quasi-metric measure space with a doubling measure and $p(\cdot)$ is log-Hölder continuous.

On the other hand, the theory of classical real Hardy spaces $H^{p}\left(\mathbf{R}^{n}\right)$ was first introduced by Stein and Weiss [57] in the early 1960s, and then was systematically developed by Fefferman and Stein [27]. It is well known that the Hardy space $H^{p}\left(\mathbf{R}^{n}\right)$ is a suitable substitute of the Lebesgue space $L^{p}\left(\mathbf{R}^{n}\right)$ for any $p \in(0,1]$. When $p \in(0,1]$, many well-known operators from harmonic analysis, such as Hilbert and Riesz transforms, are bounded on $H^{p}\left(\mathbf{R}^{n}\right)$, but not on $L^{p}\left(\mathbf{R}^{n}\right)$. As a generalization of classical Hardy spaces, Nakai and Sawano [45] introduced variable Hardy spaces $H^{p(\cdot)}\left(\mathbf{R}^{n}\right)$, established their atomic characterizations and investigated their dual spaces. Independently, Cruz-Uribe and Wang [19] also studied the variable Hardy spaces $H^{p(\cdot)}\left(\mathbf{R}^{n}\right)$ with $p(\cdot)$ satisfying some conditions slightly weaker than those used in [45]. Recently, Yang et al. [62, 67] established equivalent characterizations of variable Hardy spaces via Riesz transforms and intrinsic square functions. A complete real-variable theory of variable Hardy spaces over an RD-space (any metric measure space satisfying both the doubling and the reverse doubling conditions) was also established by Zhuo et al. [65]. Notice that $H^{p}\left(\mathbf{R}^{n}\right)$ is essentially associated with the Laplace operator

$$
\Delta:=\sum_{j=1}^{n} \frac{\partial^{2}}{\partial x_{j}^{2}}
$$

(see, for example, [26]). In recent years, there has been a lot of attention paid to the study of Hardy spaces associated with different operators, which has been a very active research topic in harmonic analysis; see, for example, [5, 8, 25, 26, 35, 36, 37, 38, 39, 55, 59]. In particular, Yang and Zhuo [61] introduced variable Hardy spaces $H_{L}^{p(\cdot)}\left(\mathbf{R}^{n}\right)$ associated with operators $L$ on $\mathbf{R}^{n}$, where $p(\cdot): \mathbf{R}^{n} \rightarrow(0,1]$ is a variable exponent function satisfying the globally log-Hölder continuous condition and $L$ is a linear operator on $L^{2}\left(\mathbf{R}^{n}\right)$ which generates an analytic semigroup $\left\{e^{-t L}\right\}_{t \geq 0}$ with kernels having pointwise upper bounds. As a generalization of [61], Yang et al. [60] further studied variable Hardy spaces $H_{L}^{p(\cdot)}\left(\mathbf{R}^{n}\right)$ associated with operator $L$ which satisfies the Davies-Gaffney estimates. The notion of the Davies-Gaffney estimates (or the so-called $L^{2}$ off-diagonal estimates) of the semigroup $\left\{e^{-t L}\right\}_{t>0}$ was first introduced by Gaffney [28] and Davies [21], which serves as good substitutes of the Gaussian upper bound of the associated heat kernel; see also [7] and the related references therein. These estimates are fundamental in many applications of semigroups. For example, they are the main technical tool (for the resolvent instead of the semigroup) in the proof of the Kato square root problem (see [6]). 
Very recently, under the assumptions that $L$ is a non-negative self-adjoint operator whose heat kernel having a Gaussian upper bound, Song and Yan [55] established a characterization of the Hardy space $H_{L}^{p}\left(\mathbf{R}^{n}\right)$ for any $p \in(0,1]$ via the non-tangential maximal function associated with the heat semigroup $\left\{e^{-t L}\right\}_{t>0}$ based on a subtle modification of the technique due to Calderón [9]. Furthermore, Song and Yan [56] generalized the results of [55] to metric measure spaces of homogeneous type; see [63, 66] for more generalizations of [55]. In particular, Zhuo and Yang [66] established the atomic and the several maximal function characterizations of variable Hardy spaces $H_{L}^{p(\cdot)}\left(\mathbf{R}^{n}\right)$.

To continue the study of $[60,66,56]$, in this article, we investigate the Hardy space $H_{L}^{p(\cdot)}(\mathcal{X})$ associated with a one-to-one operator $L$ of type $\omega$ in $L^{2}(\mathcal{X})$, with $\omega \in$ $[0, \pi / 2)$, which has a bounded holomorphic functional calculus and satisfies DaviesGaffney estimates (see Assumptions 2.6 and 2.7 below) on a metric measure space $(\mathcal{X}, d, \mu)$ of homogeneous type. In general, an operator $L$ satisfying Assumptions 2.6 and 2.7 is not necessarily non-negative self-adjoint. Notice that the "non-negative selfadjoint" assumption plays an important role in the known work (see, for example, $[35,39,55,56,66])$. This leads to that $L$ has the finite speed propagation property for solutions of the corresponding wave equation (see, for example, [35]), which allows one to characterize the Hardy spaces via atoms. Therefore, without the "non-negative self-adjoint" assumption, it requires us to characterize the Hardy spaces $H_{L}^{p(\cdot)}(\mathcal{X})$ via molecules instead of atoms (see Theorem 3.3 below) in this article. In Section 4, we study the dual space of $H_{L}^{p(\cdot)}(\mathcal{X})$. We point out that, although, in [60], Yang et al. also investigated the dual space of $H_{L}^{p(\cdot)}\left(\mathbf{R}^{n}\right)$, there are some methods which only work on $\mathbf{R}^{n}$ not on a metric measure space of homogeneous type. For example, in the arguments used in [60, Remark 4.6] to obtain the duality of Hardy spaces $H_{L}^{p(\cdot)}\left(\mathbf{R}^{n}\right)$, we can cover the annulus $\left(2^{j} B\right) \backslash\left(2^{j-1} B\right)$, with $j \in \mathbf{N}$, by approximately $2^{j n}$ balls of radius $r_{B}$. In general, this is not true in the case of metric measure spaces of homogeneous type. To overcome this difficulty, we make use of the property that the metric measure space $\mathcal{X}$ has a dyadic grid analogous to that of $\mathbf{R}^{n}$, which is due to Christ [11, Theorem 11] (see also Lemma 4.6 below).

Comparing the function spaces with constant exponents, we would like to point out that a main difficulty appearing in the study of variable function spaces exists in that the quasi-norm $\|\cdot\|_{L^{p(\cdot)(\mathcal{X})}}$ has no explicit and direct expression, which makes some estimates become very complicated. To overcome this difficulty, we make use of the boundedness of the Hardy-Littlewood maximal operator on $L^{p(\cdot)}(\mathcal{X})$ (see Lemmas 2.2 and 2.3 below) and borrow some ideas from the proof of [65, Proposition 2.11] (which was essentially proved by Sawano [53, Lemma 4.1] and is restated as Lemma 2.4 below). The role of Lemma 2.4 is to reduce some estimates in terms of $L^{p(\cdot)}(\mathcal{X})$ norms of some series of functions into some estimates in terms of $L^{q}(\mathcal{X})$ norms of some functions.

This article is organized as follows.

In Section 2, we first recall the notion of the variable Lebesgue space on a metric measure space of homogeneous type. Then we describe Assumptions 2.6 and 2.7 imposed on the operator $L$ considered in this article and introduce the definition of the variable Hardy space $H_{L}^{p(\cdot)}(\mathcal{X})$ in terms of the square function of the heat semigroup generated by $L$. Finally, we recall some notions and notation on the variable tent spaces $T^{p(\cdot)}\left(\mathcal{X}^{+}\right)$on $\mathcal{X}^{+}:=\mathcal{X} \times[0, \infty)$ and establish the atomic decomposition of $T^{p(\cdot)}\left(\mathcal{X}^{+}\right)$(see Proposition 2.13 below). 
In Section 3, we establish the molecular characterization of $H_{L}^{p(\cdot)}(\mathcal{X})$ (see Theorem 3.3 below). Following the strategy that used in the proof of [60, Propositions 3.10 and 3.12], we prove Theorem 3.3 by means of the atomic decomposition of the tent space $T^{p(\cdot)}\left(\mathcal{X}^{+}\right)$. The proof of Theorem 3.3 also relies on Lemmas 2.2 and 2.4 below.

In Section 4, we introduce the variable $\mathrm{BMO}$-type space $\mathrm{BMO}_{p(\cdot), L^{*}}(\mathcal{X})$ and show that the dual space of $H_{L}^{p(\cdot)}(\mathcal{X})$ is $\mathrm{BMO}_{p(\cdot), L^{*}}(\mathcal{X})$ (see Theorem 4.9 below), where $L^{*}$ denotes the adjoint operator of $L$ on $L^{2}(\mathcal{X})$. The proof of Theorem 4.9 is based on an argument used in the proofs of [36, Theorems 8.2 and 8.6] and [39, Theorem 4.1] with some ingenious modifications on the case of variable exponents. For example, it is difficult to compare $\left\|\chi_{B(x, r)}\right\|_{L^{p(\cdot)}(\mathcal{X})}$ with $\left\|\chi_{B(y, r)}\right\|_{L^{p(\cdot)}(\mathcal{X})}$ directly, where $B(x, r)$ and $B(y, t)$ denote two balls of $\mathcal{X}$ with $x, y \in \mathcal{X}$ and $r \in(0, \infty)$. To overcome this difficulty, we make full use of Lemma 2.2 below and establish a relationship between two quasi-norms $\|\cdot\|_{L^{p(\cdot)}(\mathcal{X})}$, respectively, corresponding to two different balls (see Remark 4.8 below for more details).

In Section 5, if $L$ is a non-negative self-adjoint operator whose heat kernel has a Gaussian upper bound, we establish the non-tangential and the radial maximal function characterizations of $H_{L}^{p(\cdot)}(\mathcal{X})$ (see Theorem 5.7 below). The proof of Theorem 5.7 is based on the atomic characterization of $H_{L}^{p(\cdot)}(\mathcal{X})$ (see Proposition 5.12 below) and some known results from Song and Yan [56].

We end this section by making some conventions on notation. In this article, we always let $(\mathcal{X}, d, \mu)$ be a metric measure space of homogeneous type. For any $p \in(0, \infty)$ and any measurable subset $E$ of $\mathcal{X}$, let $L^{p}(E)$ be the set of all measurable functions $f$ on $\mathcal{X}$ such that

$$
\|f\|_{L^{p}(E)}:=\left[\int_{E}|f(x)|^{p} d \mu(x)\right]^{1 / p}<\infty .
$$

We denote by $C$ a positive constant which is independent of the main parameters, but it may vary from line to line. We also use $C_{(\alpha, \beta, \ldots)}$ to denote a positive constant depending on the parameters $\alpha, \beta, \ldots$ The symbol $f \lesssim g$ means that $f \leq C g$. If $f \lesssim g$ and $g \lesssim f$, we then write $f \sim g$. Let $\mathbf{N}:=\{1,2, \ldots\}$ and $\mathbf{Z}_{+}:=\mathbf{N} \cup\{0\}$. For any $\alpha \in(0, \infty)$ and $x \in \mathcal{X}$, denote by $\Gamma_{\alpha}(x)$ the cone of aperture $\alpha$ with vertex $x \in \mathcal{X}$, namely,

$$
\Gamma_{\alpha}(x):=\{(y, t) \in \mathcal{X} \times(0, \infty): d(x, y)<\alpha t\} .
$$

If $\alpha=1$, we simply write $\Gamma(x)$ instead of $\Gamma_{\alpha}(x)$. For any ball

$$
B:=B\left(x_{B}, r_{B}\right):=\left\{y \in \mathcal{X}: d(x, y)<r_{B}\right\} \subset \mathcal{X}
$$

with $x_{B} \in \mathcal{X}$ and $r_{B} \in(0, \infty), \alpha \in(0, \infty)$ and $j \in \mathbf{N}$, we let $\alpha B:=B\left(x_{B}, \alpha r_{B}\right)$,

$$
U_{0}(B):=B \quad \text { and } \quad U_{j}(B):=\left(2^{j} B\right) \backslash\left(2^{j-1} B\right) .
$$

For any subsets $E, F \subset \mathcal{X}$, let

$$
\operatorname{dist}(E, F):=\inf \{d(x, y): x \in E, y \in F\} .
$$

For any $p \in[1, \infty], p^{\prime}$ denotes its conjugate number, namely, $1 / p+1 / p^{\prime}=1$.

\section{Preliminaries}

In this section, we first recall the definition of metric measure spaces of homogeneous type. Then we describe two assumptions imposed on the operator $L$ studied 
in this article. Finally, we introduce the variable Hardy spaces $H_{L}^{p(\cdot)}(\mathcal{X})$ associated with $L$.

Let $(\mathcal{X}, d)$ be a metric space equipped with a non-negative doubling Borel measure $\mu$. In what follows, for any $x \in \mathcal{X}$ and $r \in(0, \infty)$, denote by

$$
V(x, r):=\mu(B(x, r)),
$$

the volume of the open ball $B(x, r)$. Recall that a measure $\mu$ is said to be doubling if there exists a positive constant $C$ such that, for any $x \in \mathcal{X}$ and $r \in(0, \infty)$,

$$
V(x, 2 r) \leq C V(x, r) .
$$

Then the triple $(\mathcal{X}, d, \mu)$ is called a metric measure space of homogeneous type in the sense of Coifman and Weiss [12, 13]. From the doubling property (2.1), we deduce that there exist positive constants $C$ and $D$ such that, for any $\lambda \in[1, \infty), x \in \mathcal{X}$ and $r \in(0, \infty)$,

$$
V(x, \lambda r) \leq C \lambda^{D} V(x, r) .
$$

There also exists a positive constant $C$ such that, for any $x, y \in \mathcal{X}$ and $r \in(0, \infty)$,

$$
V(y, r) \leq C\left[1+\frac{d(x, y)}{r}\right]^{D} V(x, r) .
$$

Indeed, property (2.3) is a direct consequence of the triangle inequality of the metric $d$ and the strong homogeneity (2.2).

2.1. Variable Lebesgue spaces. Let $(\mathcal{X}, d, \mu)$ be a metric measure space of homogeneous type. Define $\mathcal{P}(\mathcal{X})$ to be the set of all the measurable functions $p(\cdot): \mathcal{X} \rightarrow(0, \infty)$ satisfying

$$
p_{-}:=\operatorname{essinf}_{x \in \mathcal{X}} p(x)>0 \quad \text { and } \quad p_{+}:=\operatorname{ess}_{\sup } \operatorname{su\mathcal {X}}_{x} p(x)<\infty .
$$

Then a function $p(\cdot) \in \mathcal{P}(\mathcal{X})$ is called a variable function on $\mathcal{X}$.

For any $p(\cdot) \in \mathcal{P}(\mathcal{X})$, the variable Lebesgue space $L^{p(\cdot)}(\mathcal{X})$ is defined to be the space of all measurable functions $f$ satisfying (1.1) for some $\lambda \in(0, \infty)$, equipped with the Luxemburg (quasi-)norm

$$
\|f\|_{L^{p(\cdot)}(\mathcal{X})}:=\inf \left\{\lambda \in(0, \infty): \int_{\mathcal{X}}\left[\frac{|f(x)|}{\lambda}\right]^{p(x)} d \mu(x) \leq 1\right\} .
$$

For more properties of the variable Lebesgue spaces, we refer the reader to $[17,23]$.

Remark 2.1. Let $p(\cdot) \in \mathcal{P}(\mathcal{X})$.

(i) For any $\lambda \in \mathbf{C}$ and $f \in L^{p(\cdot)}(\mathcal{X}),\|\lambda f\|_{L^{p(\cdot)}(\mathcal{X})}=|\lambda|\|f\|_{L^{p(\cdot)}(\mathcal{X})}$. In particular, if $p_{-} \in[1, \infty)$, then $\|\cdot\|_{L^{p(\cdot)}(\mathcal{X})}$ is a norm, namely, for any $f, g \in L^{p(\cdot)}(\mathcal{X})$,

$$
\|f+g\|_{L^{p(\cdot)}(\mathcal{X})} \leq\|f\|_{L^{p(\cdot)}(\mathcal{X})}+\|g\|_{L^{p(\cdot)}(\mathcal{X})} .
$$

(ii) If $p(\cdot) \equiv p \in(0, \infty)$ is a constant exponent, we find that $L^{p(\cdot)}(\mathcal{X})$ is just the classical Lebesgue space $L^{p}(\mathcal{X})$, which is defined to be the set of all measurable functions $f$ on $(\mathcal{X}, d, \mu)$ such that

$$
\|f\|_{L^{p}(\mathcal{X})}:=\left[\int_{\mathcal{X}}|f(x)|^{p} d \mu(x)\right]^{1 / p}<\infty .
$$

(iii) By (2.5), it is easy to see that, for any $f \in L^{p(\cdot)}(\mathcal{X})$ and $s \in(0, \infty)$,

$$
\left\||f|^{s}\right\|_{L^{p(\cdot)}(\mathcal{X})}=\|f\|_{L^{s p(\cdot)(\mathcal{X})}}^{s} .
$$


(iv) Let $p_{-} \in(1, \infty)$. Then, by [34, p. 90], we find that, for any $f \in L^{p(\cdot)}(\mathcal{X})$ and $g \in L^{p^{*}(\cdot)}(\mathcal{X})$

$$
\int_{\mathcal{X}}|f(x) g(x)| d \mu(x) \leq 2\|f\|_{L^{p(\cdot)}(\mathcal{X})}\|g\|_{L^{p^{*}(\cdot)}(\mathcal{X})},
$$

here and hereafter, $p^{*}(\cdot)$ denotes the dual variable exponent of $p(\cdot)$, which is defined by setting, for any $x \in \mathcal{X}$,

$$
\frac{1}{p(x)}+\frac{1}{p^{*}(x)}=1 \text {. }
$$

(v) Let $p_{-} \in(1, \infty)$. Then, by [65, Lemma 2.9] (see also [17, Theorem 2.34] and [23, Corollary 3.2.14]), we know that, for any $f \in L^{p(\cdot)}(\mathcal{X})$,

$$
\frac{1}{2}\|f\|_{L^{p(\cdot)}(\mathcal{X})} \leq \sup _{\left\{g \in L^{p^{*}(\cdot)}(\mathcal{X}):\|g\|_{L^{p^{*}(\cdot)}(\mathcal{X})} \leq 1\right\}} \int_{\mathcal{X}}|f(x) g(x)| d \mu(x) \leq 2\|f\|_{L^{p(\cdot)}(\mathcal{X})} .
$$

(vi) By an argument similar to that used in the proof of [17, Proposition 2.21], we find that, for any non-trivial function $f \in L^{p(\cdot)}(\mathcal{X})$,

$$
\int_{\mathcal{X}}\left[\frac{|f(x)|}{\|f\|_{L^{p(\cdot)}(\mathcal{X})}}\right]^{p(x)} d x=1 .
$$

Recall that a variable function $p(\cdot) \in \mathcal{P}(\mathcal{X})$ is said to be locally log-Hölder continuous if there exists a positive constant $c_{\log }$ such that, for any $x, y \in \mathcal{X}$,

$$
|p(x)-p(y)| \leq \frac{c_{\log }}{\log (e+1 / d(x, y))},
$$

and that $p(\cdot)$ is said to satisfy the log-Hölder decay condition with the basepoint $x_{0} \in \mathcal{X}$ if there exist some $p_{\infty} \in \mathbf{R}$ and a positive constant $c_{\infty}$ such that, for any $x \in \mathcal{X}$,

$$
\left|p(x)-p_{\infty}\right| \leq \frac{c_{\infty}}{\log \left(e+d\left(x, x_{0}\right)\right)}
$$

We say that $p(\cdot)$ is $\log$-Hölder continuous, denoted by $p(\cdot) \in C^{\log }(\mathcal{X})$, if $p(\cdot)$ satisfies both (2.6) and (2.7). By [65, Remark 2.4], we know that $p(\cdot) \in C^{\log }(\mathcal{X})$ is equivalent to that $p^{*}(\cdot) \in C^{\log }(\mathcal{X})$. In what follows, we always fix the basepoint $x_{0} \in \mathcal{X}$, which plays the same role as the origin of $\mathbf{R}^{n}$ does.

Recall that the central Hardy-Littlewood maximal operator $M$ is defined by setting, for all $f \in L_{\text {loc }}^{1}(\mathcal{X})$ and $x \in \mathcal{X}$,

$$
M(f)(x):=\sup _{r \in(0, \infty)} \frac{1}{V(x, r)} \int_{B(x, r)}|f(y)| d \mu(y) .
$$

The following lemma establishes the boundedness of $M$ on $L^{p(\cdot)}(\mathcal{X})$, which is just [2, Corollary 1.8] (see also [34, Theorem 4.3]).

Lemma 2.2. [2] Let $p(\cdot) \in C^{\log }(\mathcal{X})$ with $1<p_{-} \leq p_{+}<\infty$. Then there exists a positive constant $C$ such that, for any $f \in L^{p(\cdot)}(\mathcal{X})$,

$$
\|M(f)\|_{L^{p(\cdot)}(\mathcal{X})} \leq C\|f\|_{L^{p(\cdot)}(\mathcal{X})} .
$$

The following Fefferman-Stein vector-valued inequality of $\mathcal{M}$ on $L^{p(\cdot)}(\mathcal{X})$ is just [65, Theorem 2.7]. 
Lemma 2.3. [65] Let $p(\cdot) \in C^{\log }(\mathcal{X})$ with $1<p_{-} \leq p_{+}<\infty$. Then, for any given $q \in(1, \infty]$, there exists a positive constant $C$ such that, for any sequence $\left\{f_{j}\right\}_{j \in \mathbf{N}}$ of measurable functions, it holds true that

$$
\left\|\left\{\sum_{j=1}^{\infty}\left[M\left(f_{j}\right)\right]^{q}\right\}^{\frac{1}{q}}\right\|_{L^{p(\cdot)}(\mathcal{X})} \leq C\left\|\left[\sum_{j=1}^{\infty}\left|f_{j}\right|^{q}\right]^{\frac{1}{q}}\right\|_{L^{p(\cdot)}(\mathcal{X})},
$$

where, when $q=\infty$, it is understood that (2.9) means

$$
\left\|\sup _{j \in \mathbf{N}} M\left(f_{j}\right)\right\|_{L^{p(\cdot)}(\mathcal{X})} \leq C\left\|\sup _{j \in \mathbf{N}}\left|f_{j}\right|\right\|_{L^{p(\cdot)}(\mathcal{X})} .
$$

If $p(\cdot) \equiv p \in(1, \infty)$ is a constant exponent, the conclusion of Lemma 2.3 was proved in [30, Theorem 1.2].

The following lemma is a slight variant of [65, Proposition 2.11], which plays a key role in the proofs of the main results of this article.

Lemma 2.4. Let $\kappa \in[1, \infty), p(\cdot) \in C^{\log }(\mathcal{X}), p:=\min \left\{p_{-}, 1\right\}$ and $q \in[1, \infty] \cap$ $\left(p_{+}, \infty\right]$, where $p_{-}$and $p_{+}$are as in (2.4). Then there exists a positive constant $C$ such that, for any sequence $\left\{B_{j}\right\}_{j \in \mathbf{N}}$ of balls in $\mathcal{X},\left\{\lambda_{j}\right\}_{j \in \mathbf{N}} \subset \mathbf{C}$ and functions $\left\{a_{j}\right\}_{j \in \mathbf{N}}$ satisfying that, for any $j \in \mathbf{N}$, supp $a_{j} \subset \kappa B_{j}$ and $\left\|a_{j}\right\|_{L^{q}(\mathcal{X})} \leq\left[\mu\left(B_{j}\right)\right]^{1 / q}$,

$$
\left\|\left(\sum_{j=1}^{\infty}\left|\lambda_{j} a_{j}\right|^{\underline{p}}\right)^{\frac{1}{\underline{p}}}\right\|_{L^{p(\cdot)(\mathcal{X})}} \leq C \kappa^{D\left(\underline{\underline{p}}-\frac{1}{q}\right)}\left\|\left(\sum_{j=1}^{\infty}\left|\lambda_{j} \chi_{B_{j}}\right|^{\underline{p}}\right)^{\frac{1}{\underline{p}}}\right\|_{L^{p(\cdot)(\mathcal{X})}} .
$$

Proof. From (iii) and (v) of Remark 2.1, we deduce that there exists a function $g \in L^{\left(\frac{p(\cdot)}{\underline{p}}\right)^{*}}(\mathcal{X})$ with $\|g\|_{L}^{\left(\frac{p(\cdot)}{\underline{p}}\right)^{*}(\mathcal{X})} \leq 1$ such that

$$
\begin{aligned}
\left\|\left(\sum_{j=1}^{\infty}\left|\lambda_{j} a_{j}\right| \underline{\underline{p}}\right)^{\underline{\underline{p}}}\right\|_{L^{p(\cdot)(\mathcal{X})}} & =\left\|\sum_{j=1}^{\infty}\left|\lambda_{j} a_{j}\right| \underline{\underline{p}}\right\|_{L^{\frac{p}{\underline{p}(\cdot)}}(\mathcal{X})}^{\underline{\underline{p}}} \\
& \lesssim\left[\int_{\mathcal{X}} \sum_{j=1}^{\infty}\left|\lambda_{j} a_{j}\right| \underline{p}|g(x)| d \mu(x)\right]^{\underline{\underline{p}}} .
\end{aligned}
$$

Since $\underline{p}:=\min \left\{p_{-}, 1\right\}$ and $q \in[1, \infty] \cap\left(p_{+}, \infty\right)$, it follows that $q>\underline{p}$. Moreover, by the Hölder inequality, (2.2) and Lemma 2.1(iv), we find that

$$
\begin{aligned}
& \int_{\mathcal{X}} \sum_{j=1}^{\infty}\left|\lambda_{j} a_{j}\right| \underline{p}|g(x)| d \mu(x) \\
& \leq \sum_{j=1}^{\infty}\left|\lambda_{j}\right| \underline{\underline{p}}\left[\int_{\kappa B_{j}}\left|a_{j}(x)\right|^{q} d \mu(x)\right]^{\underline{p} / q}\left[\int_{\kappa B_{j}}|g(x)|^{(\underline{\underline{q}})^{\prime}} d \mu(x)\right]^{1-\underline{p} / q} \\
& \left.\leq \sum_{j=1}^{\infty}\left|\lambda_{j}\right| \underline{p}\left[\mu\left(B_{j}\right)\right]\right]^{\underline{p} / q}\left[\mu\left(\kappa B_{j}\right)\right]^{1-\underline{p} / q}\left[\frac{1}{\mu\left(\kappa B_{j}\right)} \int_{\kappa B_{j}}|g(x)|^{\left(\underline{q}^{\underline{q}}\right)^{\prime}} d \mu(x)\right]^{1-\underline{p} / q} \\
& \lesssim \sum_{j=1}^{\infty}\left|\lambda_{j}\right| \underline{p} \mu\left(B_{j}\right) \kappa^{D(1-\underline{p} / q)}\left[\inf _{x \in B_{j}} M\left(|g|^{\left(\frac{q}{p}\right)^{\prime}}\right)(x)\right]^{1 /(\underline{q})^{\prime}}
\end{aligned}
$$




$$
\begin{aligned}
& \lesssim \kappa^{D(1-\underline{p} / q)} \int_{\mathcal{X}} \sum_{j=1}^{\infty}\left|\lambda_{j}\right| \underline{p}_{B_{j}}(x)\left[M\left(|g|^{\left(\frac{q}{\underline{p}}\right)^{\prime}}\right)(x)\right]^{1 /\left(\frac{q}{\underline{p}}\right)^{\prime}} d \mu(x) \\
& \lesssim \kappa^{D(1-\underline{p} / q)}\left\|\left.\sum_{j=1}^{\infty}\left|\lambda_{j} \chi_{B_{j}}\right|\right|_{L^{\frac{p}{\underline{p}}}}\right\|_{(\mathcal{X})}\left\|\left[M\left(|g|^{\left(\frac{q}{\underline{p}}\right)^{\prime}}\right)\right]^{1 /\left(\frac{q}{\underline{p}}\right)^{\prime}}\right\|_{L^{\left(\frac{p(\cdot)}{\underline{p}}\right)^{*}(\mathcal{X})}} .
\end{aligned}
$$

By this, (2.11), the fact that

$$
\left(\frac{q}{\underline{p}}\right)^{\prime}<\left\{\left(\frac{p(\cdot)}{\underline{p}}\right)^{*}\right\}_{-} \Longleftrightarrow \frac{q}{\underline{p}}>\frac{p_{+}}{\underline{p}} \Longleftrightarrow q>p_{+},
$$

Remark 2.1(iii) and Lemma 2.2, we obtain (2.10), which completes the proof of Lemma 2.4.

Remark 2.5. In particular, if $\kappa=1$, Lemma 2.4 is just [65, Proposition 2.11]. Moreover, if $(\mathcal{X}, d, \mu):=\left(\mathbf{R}^{n},|\cdot|, d x\right)$ is the classical Euclidean space, then $\kappa=1$ and hence the conclusion of Lemma 2.4 was proved by Sawano in [53, Lemma 4.1] (see also [45, Theorem 4.6] of Nakai and Sawano).

2.2. Two assumptions on the considered operator $\boldsymbol{L}$. We first recall some knowledge about bounded holomorphic functional calculi introduced by McIntosh [44] (see also [3]).

For any $\omega \in[0, \pi)$, the closed and open $\omega$ sectors, $S_{\omega}$ and $S_{\omega}^{0}$, are defined, respectively, by setting

$S_{\omega}:=\{z \in \mathbf{C}:|\arg z| \leq \omega\} \cup\{(0,0)\} \quad$ and $\quad S_{\omega}^{0}:=\{z \in \mathbf{C} \backslash\{(0,0)\}:|\arg z|<\omega\}$.

Let $(\mathcal{X}, d, \mu)$ be a space of homogeneous type. A closed and densely defined operator $L$ on $L^{2}(\mathcal{X})$ is said to be of type $\omega$ if

(i) $\sigma(L) \subset S_{\omega}$, where $\sigma(L)$ denotes the spectrum of $L$,

(ii) for any $\theta \in(\omega, \pi)$, there exists a positive constant $C_{(\theta)}$ such that, for any $z \in \mathbf{C} \backslash S_{\theta}$,

$$
|z|\left\|(z I-L)^{-1}\right\|_{\mathcal{L}\left(L^{2}(\mathcal{X})\right)} \leq C_{(\theta)} .
$$

Here and hereafter, $\mathcal{L}\left(L^{2}(\mathcal{X})\right)$ denotes the set of all continuous linear operators from $L^{2}(\mathcal{X})$ to itself and, for any $T \in \mathcal{L}\left(L^{2}(\mathcal{X})\right)$, its operator norm is denoted by $\|T\|_{\mathcal{L}\left(L^{2}(\mathcal{X})\right)}$.

For any $\mu \in(0, \pi)$, define

$$
H_{\infty}\left(S_{\mu}^{0}\right):=\left\{f: S_{\mu}^{0} \rightarrow \mathbf{C} \text { is holomorphic and }\|f\|_{L^{\infty}\left(S_{\mu}^{0}\right)}<\infty\right\}
$$

and

$$
\Psi\left(S_{\mu}^{0}\right):=\left\{f \in H_{\infty}\left(S_{\mu}^{0}\right): \exists \alpha, C \in(0, \infty) \text { such that }|f(z)| \leq \frac{C|z|^{\alpha}}{1+|z|^{2 \alpha}}, \forall z \in S_{\mu}^{0}\right\} .
$$

Let $\omega \in[0, \pi)$ and $L$ be a one-to-one operator of type $\omega$ on $L^{2}(\mathcal{X})$. For any $\psi \in \Psi\left(S_{\mu}^{0}\right)$ with $\mu \in(\omega, \pi)$, the operator $\psi(L) \in \mathcal{L}\left(L^{2}(\mathcal{X})\right)$ is defined by setting

$$
\psi(L):=\int_{\gamma} \psi(\xi)(\xi I-L)^{-1} d \xi
$$

where $\gamma:=\left\{r e^{i \nu}: r \in(0, \infty)\right\} \cup\left\{r e^{-i \nu}: r \in(0, \infty)\right\}$, for any given $\nu \in(\omega, \mu)$, is a curve consisting of two rays parameterized anti-clockwise. By (2.12) and [3, Lecture 2], we know that the integral in (2.13) is absolutely convergent in $L^{2}(\mathcal{X})$ and 
the definition of $\psi(L)$ is independent of the choice of $\nu \in(\omega, \mu)$. Moreover, it is well known that the above holomorphic functional calculus defined on $\Psi\left(S_{\mu}^{0}\right)$ can be extended to $H_{\infty}\left(S_{\mu}^{0}\right)$ by a limiting procedure (see [44]). Let $0 \leq \omega<\mu<\pi$. Recall that the operator $L$ is said to have a bounded holomorphic functional calculus on $L^{2}(\mathcal{X})$ if there exists a positive constant $C_{(\mu, \omega)}$, depending on $\mu$ and $\omega$, such that, for any $\psi \in H_{\infty}\left(S_{\mu}^{0}\right)$,

$$
\|\psi(L)\|_{\mathcal{L}\left(L^{2}(\mathcal{X})\right)} \leq C_{(\mu, \omega)}\|\psi\|_{L^{\infty}\left(S_{\mu}^{0}\right)} .
$$

By [3, Theorem F], we know that, if (2.14) holds true for some $\mu \in(\omega, \pi)$, then it also holds true for all $\mu \in(\omega, \pi)$. As was pointed out in [5, Lecture 4], operators, which have bounded holomorphic functional calculi, include positive self-adjoint operators and maximal accretive operators on $L^{2}(\mathcal{X})$.

Let $L$ be a one-to-one operator of type $\omega$ in $L^{2}(\mathcal{X})$ with $\omega \in[0, \pi / 2)$. Then it follows from [50, Theorem 1.45] that $L$ generates a bounded holomorphic semigroup $\left\{e^{-z L}\right\}_{z \in S_{\pi / 2-\omega}^{0}}$ on the open sector $S_{\pi / 2-\omega}^{0}$.

We now make the following two assumptions on the operator $L$, which are used through the whole article.

Assumption 2.6. $L$ is a one-to-one operator of type $\omega$ in $L^{2}(\mathcal{X})$, with $\omega \in$ $[0, \pi / 2)$, and has a bounded holomorphic functional calculus on $L^{2}(\mathcal{X})$.

Assumption 2.7. The semigroup $\left\{e^{-t L}\right\}_{t>0}$ generated by $L$ satisfies the DaviesGaffney estimates, namely, there exist positive constants $C$ and $c$ such that, for any closed subsets $E$ and $F$ of $\mathcal{X}$ and $f \in L^{2}(\mathcal{X})$ with supp $f \subset E$,

$$
\left\|e^{-t L}(f)\right\|_{L^{2}(F)} \leq C e^{-c \frac{[\mathrm{dist}(E, F)]^{2}}{t}}\|f\|_{L^{2}(E)} .
$$

Examples of operators which satisfy Assumptions 2.6 and 2.7 include second order divergence form elliptic operators on $\mathbf{R}^{n}$, (magnetic) Schrödinger operators with non-negative potentials on $\mathbf{R}^{n}$ and Laplace-Beltrami operators on all complete Riemannian manifolds; see, for example, [36, 20, 28].

Remark 2.8. (i) Let $L$ satisfy Assumptions 2.6 and 2.7, and $L^{*}$ be the adjoint operator of $L$ on $L^{2}(\mathcal{X})$. Then, by [40, Theorems 5.30 and 6.22 of Chapter Three], $L^{*}$ is also a one-to-one operator of type $\omega$ on $L^{2}(\mathcal{X})$. From [31, Lemma 2.6.2], it follows that, for any $k \in \mathbf{Z}_{+}$and $t \in(0, \infty),\left[(t L)^{k} e^{-t L}\right]^{*}=\left(t L^{*}\right)^{k} e^{-t L^{*}}$. Notice that the semigroup $e^{-z L}$ is holomorphic in $S_{\pi / 2-\omega}^{0}$. From this, Assumption 2.7 and an argument similar to that used in the proof of [35, Proposition 3.1], we deduce that, for any $k \in \mathbf{Z}_{+}$, the family $\left\{(t L)^{k} e^{-t L}\right\}_{t>0}$ of operators also satisfies the DaviesGaffney estimates (2.15).

(ii) We point out that the assumption that $L$ is one-to-one is necessary for the bounded holomorphic functional calculus on $L^{2}(\mathcal{X})$ (see $\left.[44,3]\right)$. By [15, Theorem 2.3], we further know that, if $T$ is a one-to-one operator of type $\omega$ on $L^{2}(\mathcal{X})$, then $T$ has dense domain and dense range.

Assume that the operator $L$ satisfies Assumptions 2.6 and 2.7. For any $k \in \mathbf{N}$, the square function $S_{L, k}$ associated with $L$ is defined by setting, for any $f \in L^{2}(\mathcal{X})$ and $x \in \mathcal{X}$

$$
S_{L, k}(f)(x):=\left[\int_{0}^{\infty} \int_{B(x, t)}\left|\left(t^{2} L\right)^{k} e^{-t^{2} L}(f)(y)\right|^{2} \frac{d \mu(y) d t}{V(x, t) t}\right]^{1 / 2} .
$$

In particular, when $k=1$, we write $S_{L}$ instead of $S_{L, k}$. 
We observe that, for any $k \in \mathbf{N}, S_{L, k}$ is bounded on $L^{2}(\mathcal{X})$. Indeed, from the Fubini theorem, (2.3) and [3, Theorem F], we deduce that, for any $f \in L^{2}(\mathcal{X})$,

$$
\begin{aligned}
\left\|S_{L, k}(f)\right\|_{L^{2}(\mathcal{X})}^{2} & =\int_{\mathcal{X}} \int_{0}^{\infty} \int_{B(x, t)}\left|\left(t^{2} L\right)^{k} e^{-t^{2} L}(f)(y)\right|^{2} \frac{d \mu(y) d t}{V(x, t) t} d \mu(x) \\
& \lesssim \int_{0}^{\infty} \int_{\mathcal{X}}\left|\left(t^{2} L\right)^{k} e^{-t^{2} L}(f)(y)\right|^{2} d \mu(y) \frac{d t}{t} \lesssim\|f\|_{L^{2}(\mathcal{X})}^{2}
\end{aligned}
$$

where the implicit positive constant is independent of $f$.

We now introduce the variable Hardy spaces associated with the operator $L$.

Definition 2.9. Let $p(\cdot) \in C^{\log }(\mathcal{X})$ satisfy $p_{+} \in(0,1]$ and $L$ be an operator satisfying Assumptions 2.6 and 2.7. The variable Hardy space $H_{L}^{p(\cdot)}(\mathcal{X})$ is defined to be the completion of the space

$$
\left\{f \in L^{2}(\mathcal{X}):\left\|S_{L}(f)\right\|_{L^{p(\cdot)}(\mathcal{X})}<\infty\right\}
$$

with respect to the quasi-norm

$$
\|f\|_{H_{L}^{p(\cdot)}(\mathcal{X})}:=\left\|S_{L}(f)\right\|_{L^{p(\cdot)}(\mathcal{X})}=\inf \left\{\lambda \in(0, \infty): \int_{\mathcal{X}}\left[\frac{S_{L}(f)(x)}{\lambda}\right]^{p(x)} d \mu(x) \leq 1\right\} .
$$

Remark 2.10. (i) In particular, when $p(\cdot) \equiv p \in(0,1]$ is a constant, $H_{L}^{p(\cdot)}(\mathcal{X})$ was introduced in [8] as a special case and also studied in [24].

(ii) If $(\mathcal{X}, d, \mu):=\left(\mathbf{R}^{n},|\cdot|, d x\right)$ is the classical Euclidean space, the variable Hardy space $H_{L}^{p(\cdot)}(\mathcal{X})$ was studied in [60].

2.3. Variable tent spaces. In this subsection, we first introduce some notions and notation on variable tent spaces $T^{p(\cdot)}\left(\mathcal{X}^{+}\right)$. Then we establish an atomic decomposition of $T^{p(\cdot)}\left(\mathcal{X}^{+}\right)$. Here and hereafter, we always let $\mathcal{X}^{+}:=\mathcal{X} \times(0, \infty)$.

Definition 2.11. Let $p(\cdot) \in \mathcal{P}(\mathcal{X})$. The variable tent space $T^{p(\cdot)}\left(\mathcal{X}^{+}\right)$is defined to be the space of all measurable functions $f$ such that $\|f\|_{T^{p(\cdot)}\left(\mathcal{X}^{+}\right)}:=\|A(f)\|_{L^{p(\cdot)}(\mathcal{X})}<$ $\infty$, where, for any $x \in \mathcal{X}$,

$$
A(f)(x):=\left[\int_{0}^{\infty} \int_{B(x, t)}|f(y, t)|^{2} \frac{d \mu(y) d t}{V(x, t) t}\right]^{1 / 2} .
$$

In particular, if $p(\cdot) \equiv p \in(0, \infty)$ is a constant exponent, we simply write $T^{p}\left(\mathcal{X}^{+}\right)$ instead of $T^{p(\cdot)}\left(\mathcal{X}^{+}\right)$. We point out that the tent space $T^{p}\left(\mathcal{X}^{+}\right)$was studied in [4].

The following lemma is just [4, Proposition 3.10].

Lemma 2.12. [4] Let $p \in(1, \infty)$. Then, for any $f \in T^{p}\left(\mathcal{X}^{+}\right)$and $g \in T^{p^{\prime}}\left(\mathcal{X}^{+}\right)$, the pairing

$$
\langle f, g\rangle:=\int_{0}^{\infty} \int_{\mathcal{X}} f(x, t) \overline{g(x, t)} d \mu(x) \frac{d t}{t}
$$

realizes $T^{p^{\prime}}\left(\mathcal{X}^{+}\right)$as the dual of $T^{p}\left(\mathcal{X}^{+}\right)$, up to equivalent norms, where $1 / p+1 / p^{\prime}=1$.

For any $\alpha \in(0, \infty)$ and $x \in \mathcal{X}$, let $\Gamma_{\alpha}(x)$ be as in (1.2). Then, for any closed subset $E \subset \mathcal{X}$, define

$$
R_{\alpha}(E):=\bigcup_{x \in E} \Gamma_{\alpha}(x)
$$


Let $\alpha \in(0, \infty)$ and $O$ be an open subset of $\mathcal{X}$. The tent over $O$ with aperture $\alpha$ is defined by setting

$$
T_{\alpha}(O):=\left\{(y, t) \in \mathcal{X}^{+}: d\left(y, O^{\complement}\right) \geq \alpha t\right\} .
$$

If $\alpha=1$, we simply write $R(O)$ and $T(O)$, respectively, instead of $R_{1}(O)$ and $T_{1}(O)$.

Let $p(\cdot) \in \mathcal{P}(\mathcal{X})$. Recall that a measurable function $a$ on $\mathcal{X}^{+}$is called a $(p(\cdot), \infty)$ atom if there exists a ball $B \subset \mathcal{X}$ such that

(i) $\operatorname{supp} a \subset T(B)$;

(ii) for any $q \in(1, \infty)$,

$$
\|a\|_{T^{q}\left(\mathcal{X}^{+}\right)} \leq[\mu(B)]^{1 / q}\left\|\chi_{B}\right\|_{L^{p(\cdot)}(\mathcal{X})}^{-1} .
$$

In particular, if $(\mathcal{X}, d, \mu):=\left(\mathbf{R}^{n},|\cdot|, d x\right)$ is the classical Euclidean space, then $(p(\cdot), \infty)$-atom was first introduced in [67].

For any $p(\cdot) \in \mathcal{P}(\mathcal{X})$ with $0<p_{-} \leq p_{+} \leq 1$, any sequences $\left\{\lambda_{j}\right\}_{j \in \mathbf{N}} \subset \mathbf{C}$ and $\left\{B_{j}\right\}_{j \in \mathbf{N}}$ of balls in $\mathcal{X}$, let

$$
\mathcal{A}\left(\left\{\lambda_{j}\right\}_{j \in \mathbf{N}},\left\{B_{j}\right\}_{j \in \mathbf{N}}\right):=\left\|\left\{\sum_{j \in \mathbf{N}}\left[\frac{\left|\lambda_{j}\right| \chi_{B_{j}}}{\left\|\chi_{B_{j}}\right\|_{L^{p(\cdot)}(\mathcal{X})}}\right]^{p_{-}}\right\}^{\frac{1}{p_{-}}}\right\|_{L^{p(\cdot)}(\mathcal{X})} .
$$

By [65, Lemma 5.9], we know that, if $p_{+} \in(0,1]$, then, for any sequences $\left\{\lambda_{j}\right\}_{j \in \mathbf{N}} \subset$ $\mathbf{C}$ and $\left\{B_{j}\right\}_{j \in \mathbf{N}}$ of balls in $\mathcal{X}$,

$$
\sum_{j \in \mathbf{N}}\left|\lambda_{j}\right| \leq \mathcal{A}\left(\left\{\lambda_{j}\right\}_{j \in \mathbf{N}},\left\{B_{j}\right\}_{j \in \mathbf{N}}\right)
$$

The following lemma establishes the atomic decomposition of $T^{p(\cdot)}(\mathcal{X})$, which is a generalization of [67, Theorem 2.16] on the metric measure space of homogeneous type.

Proposition 2.13. Let $p \in C^{\log }(\mathcal{X})$ with $p_{+} \in(0,1]$. Then, for any $f \in$ $T^{p(\cdot)}(\mathcal{X})$, there exist $\left\{\lambda_{j}\right\}_{j \in \mathbf{N}} \subset \mathbf{C}$ and a family $\left\{a_{j}\right\}_{j \in \mathbf{N}}$ of $(p(\cdot), \infty)$-atoms such that, for almost every $(x, t) \in \mathcal{X}^{+}$,

$$
f(x, t)=\sum_{j \in \mathbf{N}} \lambda_{j} a_{j}(x, t)
$$

and

$$
\left.C^{-1}\|f\|_{T^{p(\cdot)}\left(\mathcal{X}^{+}\right)} \leq \mathcal{A}\left(\left\{\lambda_{j}\right\}_{j \in \mathbf{N}},\left\{B_{j}\right\}_{j \in \mathbf{N}}\right\}\right) \leq C\|f\|_{T^{p(\cdot)}\left(\mathcal{X}^{+}\right)},
$$

where, for any $j \in \mathbf{N}, B_{j}$ is the ball associated with $a_{j}$ and $C$ a positive constant independent of $f$. Moreover, if $f \in T^{p(\cdot)}\left(\mathcal{X}^{+}\right) \cap T^{2}\left(\mathcal{X}^{+}\right)$, then (2.21) holds true in $\operatorname{both} T^{p(\cdot)}\left(\mathcal{X}^{+}\right)$and $T^{2}\left(\mathcal{X}^{+}\right)$.

To prove Proposition 2.13, we need the following lemma which is just [43, Lemmas 2.9 and 2.16] (see also [12, Chapter III, Theorem 1.3] and [51, Lemma 2.2]).

Lemma 2.14. [43] Let $\Omega$ be a proper open subset of $\mathcal{X}$ and $\mu(\Omega)<\infty$. For any $x \in \mathcal{X}$, let $r(x):=\operatorname{dist}\left(x, \Omega^{\complement}\right) / t$. Then there exist a positive constant $M \in \mathbf{N}$ and a sequence $\left\{x_{j}\right\}_{j \in \mathbf{N}}$ of points in $\mathcal{X}$ such that, if $r_{j}:=r\left(x_{j}\right)$ for any $j \in \mathbf{N}$, then

(i) $\Omega:=\bigcup_{j \in \mathbf{N}} B\left(x_{j}, r_{j}\right)$;

(ii) $B\left(x_{i}, r_{i} / 4\right) \cap B\left(x_{j}, r_{j} / 4\right)=\emptyset$ for any $i \neq j$;

(iii) for any $j \in \mathbf{N}, \sharp\left\{i \in \mathbf{N}: B\left(x_{i}, 5 r_{i}\right) \cap B\left(x_{j}, 5 r_{j}\right) \neq \emptyset\right\} \leq M$, where $\sharp E$ denotes the cardinality of the set $E$.

Moreover, there exists a family $\left\{\phi_{j}\right\}_{j \in \mathbf{N}}$ of non-negative functions on $\mathcal{X}$ such that 
(iv) for any $j \in \mathbf{N}$, supp $\phi_{j} \subset B\left(x_{j}, 2 r_{j}\right)$;

(v) for any $j \in \mathbf{N}$ and $x \in B\left(x_{j}, r_{j}\right), \phi_{j}(x) \geq 1 / M$;

(vi) $\sum_{j \in \mathbf{N}} \phi_{j}=\chi_{\Omega}$.

We prove Proposition 2.13 by borrowing some ideas from the proofs of [51, Theorem 1.1] and [67, Theorem 2.16] (see also [59, Theorem 3.1]).

Proof of Proposition 2.13. Let $f \in T^{p(\cdot)}\left(\mathcal{X}^{+}\right)$. For any $k \in \mathbf{Z}$, let

$$
O_{k}:=\left\{x \in \mathcal{X}: A(f)(x)>2^{k}\right\} \quad \text { and } \quad O_{k}^{*}:=\left\{x \in \mathcal{X}: M\left(\chi_{O_{k}}\right)(x)>1-\gamma\right\},
$$

where $\gamma \in(0,1)$ is a constant, which is determined later, and $M$ denotes the HardyLittlewood maximal function as in (2.8). Then it is easy to see that, for any $k \in \mathbf{Z}$, $O_{k}$ is open, $O_{k+1} \subset O_{k}$ and $O_{k} \subset O_{k}^{*}$ which is guaranteed by $\gamma \in(0,1)$. By the fact that $f \in T^{p(\cdot)}\left(\mathcal{X}^{+}\right)$with $0<p_{-} \leq p_{+} \leq 1$, we find that, for any $k \in \mathbf{Z}, \mu\left(O_{k}\right)<\infty$, which, together with the boundedness of $M$ from $L^{1}(\mathcal{X})$ to the weak Lebesgue space $W L^{1}(\mathcal{X})$, further implies that $\mu\left(O_{k}^{*}\right) \lesssim \frac{1}{1-\gamma} \mu\left(O_{k}\right)<\infty$. Let $\eta \in(0,1)$ be a fixed constant. By an argument similar to that used in the proof of $[51,(2.3)]$ (see also $[59$, p. 513]), we know that there exists a $\gamma \in(0,1)$ such that

$$
\operatorname{supp} f \subset\left[\bigcup_{k \in \mathbf{Z}} T_{1-\eta}\left(O_{k}^{*}\right) \bigcup E\right] \text {, }
$$

where $E \subset \mathcal{X}^{+}$satisfies $\int_{E} \frac{d \mu(y) d t}{t}=0$.

If there exists some $k \in \mathbf{Z}$ such that $O_{k}^{*}=\mathcal{X}$, then $\mu(\mathcal{X})<\infty$. This implies that $\mathcal{X}$ is bounded, indeed, $\mathcal{X}$ is a ball (see [46, Lemma 5.1]). In this case, let $I_{k}:=\{1\}, B_{k, 1}:=\mathcal{X}$ and $\phi_{k, 1} \equiv 1$. If $O_{k}^{*}$ is a proper subset of $\mathcal{X}$ for any $k \in \mathbf{Z}$, by applying Lemma 2.14 with $\Omega:=O_{k}^{*}$ therein, we obtain a set $I_{k} \subset \mathbf{N}$ of indices, a family $\left\{B_{k, j}\right\}_{j \in I_{k}}:=\left\{B\left(x_{k, j}, 2 r_{k, j}\right)\right\}_{j \in I_{k}}$ of balls and a family $\left\{\phi_{k, j}\right\}_{j \in I_{k}}$ of functions satisfying that, for any $j \in I_{k}$, supp $\phi_{k, j} \subset B_{k, j}$ and $\sum_{j \in I_{k}} \phi_{k, j}=\chi_{O_{k}^{*}}$. Then it is easy to see that, for any $k \in \mathbf{Z}$ and $(x, t) \in \mathcal{X}^{+}$,

$$
\chi_{T_{1-\eta}\left(O_{k}^{*}\right) \backslash T_{1-\eta}\left(O_{k+1}^{*}\right)}(x, t)=\sum_{j \in I_{k}} \phi_{k, j}(x) \chi_{T_{1-\eta}\left(O_{k}^{*}\right) \backslash T_{1-\eta}\left(O_{k+1}^{*}\right)}(x, t) .
$$

From this and (2.22), we deduce that, for almost every $(x, t) \in \mathcal{X}^{+}$,

$$
\begin{aligned}
f(x, t) & =\sum_{k \in \mathbf{Z}} f(x, t) \chi_{T_{1-\eta}\left(O_{k}^{*}\right) \backslash T_{1-\eta}\left(O_{k+1}^{*}\right)}(x, t) \\
& =\sum_{k \in \mathbf{Z}} \sum_{j \in I_{k}} f(x, t) \phi_{k, j}(x, t) \chi_{T_{1-\eta}\left(O_{k}^{*}\right) \backslash T_{1-\eta}\left(O_{k+1}^{*}\right)}(x, t) .
\end{aligned}
$$

For any $k \in \mathbf{Z}$ and $j \in I_{k}$, let

$$
a_{k, j}:=2^{-k}\left\|\chi_{B_{k, j}}\right\|_{L^{p(\cdot)}(\mathcal{X})}^{-1} f \phi_{k, j} \chi_{T_{1-\eta}\left(O_{k}^{*}\right) \backslash T_{1-\eta}\left(O_{k+1}^{*}\right)}
$$

and

$$
\lambda_{k, j}:=2^{k}\left\|\chi_{B_{k, j}}\right\|_{L^{p(\cdot)}(\mathcal{X})}^{-1} .
$$

Then we have $f=\sum_{k \in \mathbf{Z}} \sum_{j \in I_{k}} \lambda_{k, j} a_{k, j}$. By an argument similar to that used in the proof of $[51,(2.4)]$ and Lemma 2.12, we conclude that, for any $k \in \mathbf{Z}$ and $j \in I_{k}$, supp $a_{k, j} \subset T\left(C_{(\eta)} B_{k, j}\right)$, where $C_{(\eta)}=2+\frac{12}{1-\eta}$, and $a_{k, j}$ is a uniform harmless positive 
constant multiple of a $(p(\cdot), \infty)$-atom. Moreover, by an argument similar to that used in the proof of [67, Theorem 2.16], we find that

$$
\mathcal{A}\left(\left\{\lambda_{k, j}\right\}_{k \in \mathbf{Z}, j \in I_{k}},\left\{C_{(\eta)} B_{k, j}\right\}_{k \in \mathbf{Z}, j \in I_{k}}\right) \lesssim\|f\|_{T^{p(\cdot)}\left(\mathcal{X}^{+}\right)},
$$

where the implicit positive constant depends on $\eta$ and $\gamma$.

Next, we prove the reverse inequality. Indeed, by the fact that $a_{k, j}$ is a $(p(\cdot), \infty)$ atom associated with the ball $C_{(\eta)} B_{k, j}$ for any $k \in \mathbf{Z}$ and $j \in I_{k}$, we know that $\operatorname{supp} A\left(a_{k, j}\right) \subset C_{(\eta)} B_{k, j}$ and, for any $q \in(1, \infty)$,

$$
\left\|A\left(a_{k, j}\right)\right\|_{L^{q}(\mathcal{X})} \lesssim\left[\mu\left(C_{(\eta)} B_{k, j}\right)\right]^{1 / q}\left\|\chi_{C(\eta) B_{k, j}}\right\|_{L^{p(\cdot)}(\mathcal{X})}^{-1} .
$$

From this, the fact that $p_{-} \in(0,1]$ and Lemma 2.4, it follows that

$$
\begin{aligned}
\|f\|_{T^{p(\cdot)}\left(\mathcal{X}^{+}\right)} & =\|A(f)\|_{L^{p(\cdot)}(\mathcal{X})} \leq\left\|\sum_{k \in \mathbf{Z}} \sum_{j \in I_{k}}\left|\lambda_{k, j}\right| A\left(a_{k, j}\right)\right\|_{L^{p(\cdot)}(\mathcal{X})} \\
& \leq\left\|\left\{\sum_{k \in \mathbf{Z}} \sum_{j \in I_{k}}\left[\left|\lambda_{k, j}\right| A\left(a_{k, j}\right)\right]^{p_{-}}\right\}^{\frac{1}{p_{-}}}\right\| \|_{L^{p(\cdot)}(\mathcal{X})} \\
& \lesssim\left\|\left\{\sum_{k \in \mathbf{Z}} \sum_{j \in I_{k}}\left[\frac{\left|\lambda_{k, j}\right|}{\left\|\chi_{C_{(\eta)} B_{k, j}}\right\|_{L^{p(\cdot)}(\mathcal{X})}} \chi_{C_{(\eta)} B_{k, j}}\right]^{p_{-}}\right\}^{\frac{1}{p_{-}}}\right\|_{L^{p(\cdot)}(\mathcal{X})} \\
& \sim \mathcal{A}\left(\left\{\lambda_{k, j}\right\},\left\{C_{(\eta)} B_{k, j}\right\}\right) .
\end{aligned}
$$

To complete the proof of Proposition 2.13, it remains to show that, if $f \in$ $T^{p(\cdot)}\left(\mathcal{X}^{+}\right) \cap T^{2}\left(\mathcal{X}^{+}\right)$, then the series

$$
f=\sum_{k \in \mathbf{Z}} \sum_{j \in I_{k}} \lambda_{k, j} a_{k, j}
$$

converges in both $T^{p(\cdot)}\left(\mathcal{X}^{+}\right)$and $T^{2}\left(\mathcal{X}^{+}\right)$. Indeed, if $f \in T^{p(\cdot)}\left(\mathcal{X}^{+}\right)$, by an argument similar to that used in (2.26), we find that, for any $N \in \mathbf{N}$,

$$
\begin{aligned}
& \left\|A\left(f-\sum_{|k|+|j| \leq N} \lambda_{k, j} a_{k, j}\right)\right\|_{L^{p(\cdot)}(\mathcal{X})} \\
& \lesssim\left\|\left\{\sum_{|k|+|j|>N}\left[\frac{\left|\lambda_{k, j}\right|}{\left\|\chi_{C_{(\eta)} B_{k, j}}\right\|_{L^{p(\cdot)}(\mathcal{X})}} \chi_{C_{(\eta)} B_{k, j}}\right]^{p_{-}}\right\}^{\frac{1}{p_{-}}}\right\|_{L^{p(\cdot)}(\mathcal{X})} .
\end{aligned}
$$

This, together with (2.25) and the dominated convergence theorem (see, for example, [23, Lemma 3.2.8]), implies that

$$
\begin{aligned}
& \lim _{N \rightarrow \infty}\left\|A\left(f-\sum_{|k|+|j| \leq N} \lambda_{k, j} a_{k, j}\right)\right\|_{L^{p(\cdot)}(\mathcal{X})} \\
& \lesssim\left\|\lim _{N \rightarrow \infty}\left\{\sum_{|k|+|j|>N}\left[\frac{\left|\lambda_{k, j}\right|}{\left\|\chi_{C_{(\eta)} B_{k, j}}\right\|_{L^{p(\cdot)}(\mathcal{X})}} \chi_{C_{(\eta)} B_{k, j}}\right]^{p_{-}}\right\}^{\frac{1}{p_{-}}}\right\|_{L^{p(\cdot)}(\mathcal{X})}=0 .
\end{aligned}
$$

Hence, (2.27) converges in $T^{p(\cdot)}\left(\mathcal{X}^{+}\right)$. 
If $f \in T^{2}\left(\mathcal{X}^{+}\right)$, by the Fubini theorem and (2.3), we obtain

$$
\begin{aligned}
& \left\|A\left(f-\sum_{|k|+|j| \leq N} \lambda_{k, j} a_{k, j}\right)\right\|_{L^{2}(\mathcal{X})} \\
& =\int_{\mathcal{X}} \int_{0}^{\infty} \int_{B(x, t)}\left|\sum_{|k|+|j|>N} \lambda_{k, j} a_{k, j}(y, t)\right|^{2} \frac{d \mu(y) d t}{V(x, t) t} d \mu(x) \\
& \lesssim \int_{0}^{\infty} \int_{\mathcal{X}} \sum_{|k|+|j|>N}\left|\lambda_{k, j} a_{k, j}(y, t)\right|^{2} \frac{d \mu(y) d t}{t} .
\end{aligned}
$$

On the other hand, from Lemma 2.14(iii), (2.23) and (2.24), we deduce that, for almost every $(y, t) \in \mathcal{X}^{+}$,

$$
\sum_{k \in \mathbf{Z}} \sum_{j \in I_{k}}\left|\lambda_{k, j} a_{k, j}(y, t)\right| \lesssim|f(y, t)| .
$$

This, combined with (2.28) and the Lebesgue dominated convergence theorem, further implies that

$$
\begin{aligned}
& \lim _{N \rightarrow \infty}\left\|A\left(f-\sum_{|k|+|j| \leq N} \lambda_{k, j} a_{k, j}\right)\right\|_{L^{2}(\mathcal{X})} \\
& \lesssim \int_{0}^{\infty} \int_{\mathcal{X}} \lim _{N \rightarrow \infty} \sum_{|k|+|j|>N}\left|\lambda_{k, j} a_{k, j}(y, t)\right|^{2} \frac{d \mu(y) d t}{t}=0 .
\end{aligned}
$$

Therefore, (2.27) converges in $T^{2}\left(\mathcal{X}^{+}\right)$. This finishes the proof of Proposition 2.13.

\section{Molecular characterization of $H_{L}^{p(\cdot)}(\mathcal{X})$}

In this section, we establish the molecular characterization of $H_{L}^{p(\cdot)}(\mathcal{X})$. We begin with recalling some notions.

Definition 3.1. Let $L$ satisfy Assumptions 2.6 and 2.7 and $p(\cdot) \in \mathcal{P}(\mathcal{X})$ with $p_{+} \in(0,1]$. Assume $M \in \mathbf{N}$ and $\varepsilon \in(0, \infty)$. A function $m \in L^{2}(\mathcal{X})$ is called a $(p(\cdot), M, \varepsilon)_{L}$-molecule if $m \in \mathcal{R}\left(L^{M}\right)$ (the range of $L^{M}$ ) and there exists a ball $B:=$ $B\left(x_{B}, r_{B}\right) \subset \mathcal{X}$ with $x_{B} \in \mathcal{X}$ and $r_{B} \in(0, \infty)$ such that, for any $k \in\{0, \ldots, M\}$ and $j \in \mathbf{Z}_{+}$,

$$
\left\|\left(r_{B}^{-2} L^{-1}\right)^{k}(m)\right\|_{L^{2}\left(U_{j}(B)\right)} \leq 2^{-j \varepsilon}\left[\mu\left(2^{j} B\right)\right]^{1 / 2}\left\|\chi_{B}\right\|_{L^{p(\cdot)}(\mathcal{X})}^{-1} .
$$

Definition 3.2. Let $L$ satisfy Assumptions 2.6 and 2.7 and $p(\cdot) \in \mathcal{P}(\mathcal{X})$ with $p_{+} \in(0,1]$. Assume $M \in \mathbf{N}$ and $\varepsilon \in(0, \infty)$. For a measurable function $f$ on $\mathcal{X}$, $f=\sum_{j=1}^{\infty} \lambda_{j} m_{j}$ is called a molecular $(p(\cdot), M, \varepsilon)$-representation of $f$ if $\left\{m_{j}\right\}_{j \in \mathbf{N}}$ is a family of $(p(\cdot), M, \varepsilon)_{L}$-molecules, the summation converges in $L^{2}(\mathcal{X})$ and $\left\{\lambda_{j}\right\}_{j \in \mathbf{N}} \subset$ C satisfies that $\mathcal{A}\left(\left\{\lambda_{j}\right\}_{j \in \mathbf{N}},\left\{B_{j}\right\}_{j \in \mathbf{N}}\right)<\infty$, where, for any $j \in \mathbf{N}, B_{j}$ is the ball associated with $m_{j}$. Let

$$
\mathbf{H}_{L, M}^{p(\cdot), \varepsilon}(\mathcal{X}):=\{f: f \text { has a molecular }(p(\cdot), M, \varepsilon) \text {-representation }\} .
$$


Then the variable molecular Hardy space $H_{L, M}^{p(\cdot), \varepsilon}(\mathcal{X})$ is defined to be the completion of $\mathbf{H}_{L, M}^{p(\cdot), \varepsilon}(\mathcal{X})$ with respect to the quasi-norm

$$
\begin{aligned}
\|f\|_{H_{L, M}^{p(\cdot), \varepsilon}(\mathcal{X})}:=\inf \left\{\mathcal{A}\left(\left\{\lambda_{j}\right\}_{j \in \mathbf{N}},\left\{B_{j}\right\}_{j \in \mathbf{N}}\right):\right. \\
\left.\quad f=\sum_{j=1}^{\infty} \lambda_{j} m_{j} \text { is a molecular }(p(\cdot), M, \varepsilon) \text {-representation }\right\},
\end{aligned}
$$

where $\mathcal{A}\left(\left\{\lambda_{j}\right\}_{j \in \mathbf{N}},\left\{B_{j}\right\}_{j \in \mathbf{N}}\right)$ is as in (2.19) and the infimum is taken over all the molecular $(p(\cdot), M, \varepsilon)$-representations of $f$ as above.

The following theorem establishes the molecular characterization of $H_{L}^{p(\cdot)}(\mathcal{X})$.

Theorem 3.3. Let $L$ satisfy Assumptions 2.6 and 2.7 and $p(\cdot) \in C^{\log }(\mathcal{X})$ with $p_{+} \in(0,1]$. Assume $M \in \mathbf{N} \cap\left(\frac{D}{2}\left(\frac{1}{p_{-}}-\frac{1}{2}\right), \infty\right)$ and $\varepsilon \in\left(\frac{D}{p_{-}}, \infty\right)$. Then $H_{L, M}^{p(\cdot), \varepsilon}(\mathcal{X})$ and $H_{L}^{p(\cdot)}(\mathcal{X})$ coincide with equivalent quasi-norms.

Remark 3.4. (i) If $(\mathcal{X}, d, \mu):=\left(\mathbf{R}^{n},|\cdot|, d x\right)$ is the classical Euclidean space, Theorem 3.3 is just [60, Theorem 3.14] and the ranges of $M$ and $\varepsilon$ coincide with those of [60, Theorem 3.14], respectively.

(ii) If $p(\cdot) \equiv p \in(0,1]$ is a constant exponent, from Theorem 3.3, we deduce that, for any given $M \in \mathbf{N} \cap \frac{n}{2}\left(\frac{1}{p}-\frac{1}{2}\right)$ and $\varepsilon \in\left(\frac{n}{p}, \infty\right), H_{L, M}^{p, \varepsilon}(\mathcal{X})$ and $H_{L}^{p}(\mathcal{X})$ coincide with equivalent quasi-norms. This is just [24, Theorem 3.15] and the ranges of $M$ and $\varepsilon$ coincide with those of [24, Theorem 3.15], respectively.

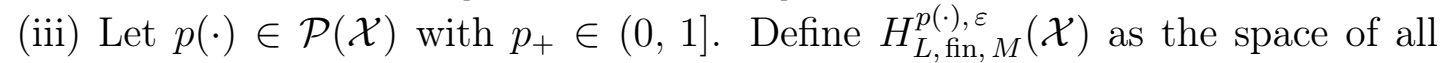

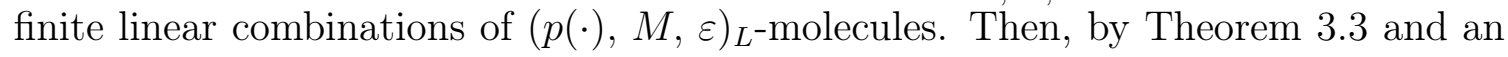
argument similar to that used in the proof of [60, Proposition 3.13], we know that, if $M \in \mathbf{N} \cap\left(\frac{D}{2}\left(\frac{1}{p_{-}}-\frac{1}{2}\right), \infty\right)$ and $\varepsilon \in\left(\frac{D}{p_{-}}, \infty\right)$, then $H_{L, \text { fin, } M}^{p(\cdot), \varepsilon}(\mathcal{X})$ is dense in $H_{L}^{p(\cdot)}(\mathcal{X})$ with respect to the quasi-norm $\|\cdot\|_{H_{L}^{p(\cdot)}(\mathcal{X})}$.

To prove Theorem 3.3, we first show that $\mathbf{H}_{L, M}^{p(\cdot), \varepsilon}(\mathcal{X}) \subset\left[H_{L}^{p(\cdot)}(\mathcal{X}) \cap L^{2}(\mathcal{X})\right]$.

Proposition 3.5. Let $L$ satisfy Assumptions 2.6 and 2.7, and $p(\cdot) \in C^{\log }(\mathcal{X})$ with $p_{+} \in(0,1]$. Assume $M \in \mathbf{N} \cap\left(\frac{D}{2}\left(\frac{1}{p_{-}}-\frac{1}{2}\right), \infty\right)$ and $\varepsilon \in\left(\frac{D}{p_{-}}, \infty\right)$. Then there exists a positive constant $C$ such that, for any $f \in \mathbf{H}_{L, M}^{p(\cdot), \varepsilon}(\mathcal{X})$,

$$
\|f\|_{H_{L}^{p(\cdot)}(\mathcal{X})} \leq C\|f\|_{H_{L, M}^{p(\cdot), \varepsilon}(\mathcal{X})} .
$$

Proof. Let $f \in \mathbf{H}_{L, M}^{p(\cdot), \varepsilon}(\mathcal{X})$. Then, by Definition 3.2, we know that there exist $\left\{\lambda_{j}\right\}_{j \in \mathbf{N}} \subset \mathbf{C}$ and a family $\left\{m_{j}\right\}_{j \in \mathbf{N}}$ of $(p(\cdot), M, \varepsilon)_{L}$-molecules, associated with balls $\left\{B_{j}\right\}_{j \in \mathbf{N}}$ of $\mathcal{X}$, such that

$$
f=\sum_{j=1}^{\infty} \lambda_{j} m_{j} \text { in } L^{2}(\mathcal{X})
$$

and

$$
\|f\|_{H_{L, M}^{p(\cdot), \varepsilon}(\mathcal{X})} \sim \mathcal{A}\left(\left\{\lambda_{j}\right\}_{j \in \mathbf{N}},\left\{B_{j}\right\}_{j \in \mathbf{N}}\right)
$$


where $\mathcal{A}\left(\left\{\lambda_{j}\right\}_{j \in \mathbf{N}},\left\{B_{j}\right\}_{j \in \mathbf{N}}\right)$ is as in (2.19). By (3.2) and (2.16), we find that

$$
\lim _{N \rightarrow \infty}\left\|S_{L}(f)-S_{L}\left(\sum_{j=1}^{N} \lambda_{j} m_{j}\right)\right\|_{L^{2}(\mathcal{X})}=0
$$

which implies that there exists a subsequence of $\left\{S_{L}\left(\sum_{j=1}^{N} \lambda_{j} m_{j}\right)\right\}_{N \in \mathbf{N}}$, without loss of generality, we may use the same notation as the original sequence such that, for almost every $x \in \mathcal{X}$

$$
S_{L}(f)(x)=\lim _{N \rightarrow \infty} S_{L}\left(\sum_{j=1}^{N} \lambda_{j} m_{j}\right)(x)
$$

Hence, for almost every $x \in \mathcal{X}$, it holds true that

$$
S_{L}(f)(x) \leq \sum_{j=1}^{\infty}\left|\lambda_{j}\right| S_{L}\left(m_{j}\right)(x)=\sum_{j=1}^{\infty} \sum_{i=0}^{\infty}\left|\lambda_{j}\right| S_{L}\left(m_{j}\right)(x) \chi_{U_{i}\left(B_{j}\right)}(x) .
$$

From this, Remark 2.1 and the fact that $p_{-} \in(0,1]$, it follows that

$$
\begin{aligned}
\left\|S_{L}(f)\right\|_{L^{p(\cdot)}(\mathcal{X})}^{p_{-}} & =\left\|\left[S_{L}(f)\right]^{p_{-}}\right\|_{L^{\frac{p(\cdot)}{p_{-}}(\mathcal{X})}} \\
& \leq \sum_{i=0}^{\infty}\left\|\sum_{j=1}^{\infty}\left|\lambda_{j}\right|^{p_{-}}\left[S_{L}\left(m_{j}\right) \chi_{U_{i}\left(B_{j}\right)}\right]^{p_{-}}\right\|_{L^{\frac{p(\cdot)}{p_{-}}(\mathcal{X})}} \\
& =\sum_{i=0}^{\infty}\left\|\left\{\sum_{j=1}^{\infty}\left|\lambda_{j}\right|^{p_{-}}\left[S_{L}\left(m_{j}\right) \chi_{U_{i}\left(B_{j}\right)}\right]^{p_{-}}\right\}^{\frac{1}{p_{-}}}\right\|_{L^{p(\cdot)}(\mathcal{X})}^{p_{-}} .
\end{aligned}
$$

To prove Proposition 3.5, we only need to show that there exist positive constants $C$ and $\theta \in\left(D\left(\frac{1}{p_{-}}-\frac{1}{2}\right), \infty\right)$ such that, for any $(p(\cdot), M, \varepsilon)_{L^{-m o l e c u l e}} m$, associated with a ball $B:=B\left(x_{B}, r_{B}\right)$ for some $x_{B} \in \mathcal{X}$ and $r_{B} \in(0, \infty)$, and $i \in \mathbf{Z}_{+}$,

$$
\left\|S_{L}(m)\right\|_{L^{2}\left(U_{i}(B)\right)} \leq C 2^{-i \theta}[\mu(B)]^{1 / 2}\left\|\chi_{B}\right\|_{L^{p(\cdot)}(\mathcal{X})}^{-1} .
$$

Indeed, if (3.5) holds true, we then find that, for any $i \in \mathbf{Z}_{+}$and $j \in \mathbf{N}$,

$$
\left\|2^{i \theta}\right\| \chi_{B_{j}}\left\|_{L^{p(\cdot)}(\mathcal{X})} S_{L}\left(m_{j}\right) \chi_{U_{i}\left(B_{j}\right)}\right\|_{L^{2}(\mathcal{X})} \lesssim\left[\mu\left(B_{j}\right)\right]^{1 / 2} .
$$

By this and Lemma 2.4, we know that

$$
\begin{aligned}
& \left\|\left\{\sum_{j=1}^{\infty}\left[\left|\lambda_{j}\right| S_{L}\left(m_{j}\right) \chi_{U_{i}\left(B_{j}\right)}\right]^{p_{-}}\right\}^{\frac{1}{p_{-}}}\right\|_{L^{p(\cdot)}(\mathcal{X})} \\
& \lesssim 2^{i D\left(\frac{1}{p_{-}}-\frac{1}{2}\right)}\left\|\left\{\sum_{j=1}^{\infty}\left[2^{-i \theta}\left\|\chi_{B_{j}}\right\|_{L^{p(\cdot)}(\mathcal{X})}^{-1}\left|\lambda_{j}\right| \chi_{B_{j}}\right]^{p_{-}}\right\}^{\frac{1}{p_{-}}}\right\|_{L^{p(\cdot)}(\mathcal{X})} \\
& \lesssim 2^{-i\left[\theta-D\left(\frac{1}{p_{-}}-\frac{1}{2}\right)\right]} \mathcal{A}\left(\left\{\lambda_{j}\right\}_{j \in \mathbf{N}},\left\{B_{j}\right\}_{j \in \mathbf{N}) .}\right.
\end{aligned}
$$


Combining (3.6), (3.4), (3.3) and the fact that $\theta>D\left(\frac{1}{p_{-}}-\frac{1}{2}\right)$, we further conclude that, for any $f \in \mathbf{H}_{L, M}^{p(\cdot), \varepsilon}(\mathcal{X}) \cap L^{2}(\mathcal{X})$,

$$
\left\|S_{L}(f)\right\|_{L^{p(\cdot)(\mathcal{X})}} \lesssim\left\{\sum_{i=0}^{\infty} 2^{-i\left[\theta-D\left(\frac{1}{p_{-}}-\frac{1}{2}\right)\right] p_{-}}\right\}^{\frac{1}{p_{-}}} \mathcal{A}\left(\left\{\lambda_{j}\right\}_{j \in \mathbf{N}},\left\{B_{j}\right\}_{j \in \mathbf{N}}\right) \lesssim\|f\|_{H_{L, M}^{p(\cdot), \varepsilon}(\mathcal{X})},
$$

which is the desired result.

Next, we prove (3.5). Indeed, when $i \in\{0, \ldots, 10\}$, by $(3.1), \varepsilon \in\left(\frac{D}{p_{-}}, \infty\right)$ and the fact that $S_{L}$ is bounded on $L^{2}(\mathcal{X})$ (see $(2.16)$ ), we obtain

$$
\left\|S_{L}(m)\right\|_{L^{2}\left(U_{i}(B)\right)} \lesssim\|m\|_{L^{2}(\mathcal{X})} \lesssim[\mu(B)]^{1 / 2}\left\|\chi_{B}\right\|_{L^{p(\cdot)}(\mathcal{X})}^{-1}
$$

When $i \in \mathbf{Z}_{+} \cap[11, \infty)$, for any given $\eta \in(0,1)$, we write

$$
\begin{aligned}
\left\|S_{L}(m)\right\|_{L^{2}\left(U_{i}(B)\right)}= & {\left[\int_{U_{i}(B)} \int_{0}^{\infty} \int_{B(x, t)}\left|t^{2} L e^{-t^{2} L}(m)(y)\right|^{2} \frac{d \mu(y) d t}{V(x, t) t} d \mu(x)\right]^{\frac{1}{2}} } \\
\leq & {\left[\int_{U_{i}(B)} \int_{0}^{2^{i \eta} r_{B}} \int_{B(x, t)}\left|t^{2} L e^{-t^{2} L}(m)(y)\right|^{2} \frac{d \mu(y) d t}{V(x, t) t} d \mu(x)\right]^{\frac{1}{2}} } \\
& +\left[\int_{U_{i}(B)} \int_{2^{i \eta_{B}}}^{\infty} \int_{B(x, t)} \cdots \frac{d \mu(y) d t}{V(x, t) t} d \mu(x)\right]^{\frac{1}{2}}=: \text { I }+ \text { II. }
\end{aligned}
$$

To estimate II, by $(2.16),(3.1)$ and the fact that $\varepsilon \in\left(\frac{D}{p_{-}}, \infty\right)$, we know that

$$
\begin{aligned}
\mathrm{II}= & {\left[\int_{U_{i}(B)} \int_{2^{i \eta} r_{B}}^{\infty} \int_{B(x, t)}\left|\left(t^{2} L\right)^{M+1} e^{-t^{2} L}\left(L^{-M}(m)\right)(y)\right|^{2}\right.} \\
& \left.\times t^{-4 M} \frac{d \mu(y) d t}{V(x, t) t} d \mu(x)\right]^{\frac{1}{2}} \\
\leq & \left(2^{i \eta} r_{B}\right)^{-2 M} r_{B}^{2 M}\left\|S_{L, M+1}\left(r_{B}^{-2 M} L^{-M}(m)\right)\right\|_{L^{2}\left(U_{i}(B)\right)} \\
\lesssim & 2^{-2 i \eta M}\left\|\left(r_{B}^{-2} L^{-1}\right)^{M}(m)\right\|_{L^{2}(\mathcal{X})} \lesssim 2^{-2 i \eta M}[\mu(B)]^{1 / 2}\left\|\chi_{B}\right\|_{L^{p(\cdot)}(\mathcal{X})}^{-1}
\end{aligned}
$$

To estimate I, for any $i \in \mathbf{Z}_{+} \cap[11, \infty)$, let

$$
S_{i}(B):=\left(2^{i+1} B\right) \backslash\left(2^{i-2} B\right) \quad \text { and } \quad \widetilde{S}_{i}(B):=\left(2^{i+2} B\right) \backslash\left(2^{i-3} B\right) .
$$

If $t \in\left(0,2^{i \eta} r_{B}\right)$ and $x \in U_{i}(B)$, then it is easy to see that $B(x, t) \subset S_{i}(B)$. From this, we deduce that

$$
\begin{aligned}
\mathrm{I} \leq & {\left[\int_{U_{i}(B)} \int_{0}^{2^{i \eta} r_{B}} \int_{S_{i}(B)}\left|t^{2} L e^{-t^{2} L}\left(m \chi_{\left[\widetilde{S}_{i}(B)\right]^{\complement}}\right)(y)\right|^{2} \frac{d \mu(y) d t}{V(x, t) t} d \mu(x)\right]^{\frac{1}{2}} } \\
& +\left[\int_{U_{i}(B)} \int_{0}^{2^{i \eta} r_{B}} \int_{B(x, t)}\left|t^{2} L e^{-t^{2} L}\left(m \chi_{\widetilde{S}_{i}(B)}\right)(y)\right|^{2} \frac{d \mu(y) d t}{V(x, t) t} d \mu(x)\right]^{\frac{1}{2}} \\
= & : \mathrm{I}_{1}+\mathrm{I}_{2} .
\end{aligned}
$$


For $\mathrm{I}_{2}$, by the boundedness of $S_{L}$ on $L^{2}(\mathcal{X})$ (see (2.16)) and (3.1), we obtain

$$
\begin{aligned}
\mathrm{I}_{2} & \leq\left\|S_{L}\left(m \chi_{\widetilde{S}_{i}(B)}\right)\right\|_{L^{2}(\mathcal{X})} \lesssim\left\|m \chi_{\widetilde{S}_{i}(B)}\right\|_{L^{2}(\mathcal{X})} \lesssim 2^{-i \varepsilon}\left[\mu\left(2^{i} B\right)\right]^{1 / 2}\left\|\chi_{B}\right\|_{L^{p(\cdot)}(\mathcal{X})}^{-1} \\
& \lesssim 2^{-i\left(\varepsilon-\frac{D}{2}\right)}[\mu(B)]^{1 / 2}\left\|\chi_{B}\right\|_{L^{p(\cdot)}(\mathcal{X})}^{-1} .
\end{aligned}
$$

For $\mathrm{I}_{1}$, by Remark 2.8(i), we know that $\left\{t L e^{-t L}\right\}_{t>0}$ satisfies the Davies-Gaffney estimates. From this and the fact that $\operatorname{dist}\left(\left[\widetilde{S}_{i}(B)\right]^{\complement}, S_{i}(B)\right) \sim 2^{i} r_{B}$, it follows that

$$
\begin{aligned}
\mathrm{I}_{1} & \lesssim\left[\int_{U_{i}(B)} \int_{0}^{2^{i \eta} r_{B}} e^{-c \frac{\left(2^{i} r_{B}\right)^{2}}{t^{2}}}\|m\|_{L^{2}(\mathcal{X})}^{2} \frac{d t}{V(x, t) t} d \mu(x)\right]^{\frac{1}{2}} \\
& \lesssim\|m\|_{L^{2}(\mathcal{X})}\left[\int_{U_{i}(B)} \int_{0}^{2^{i \eta} r_{B}}\left(\frac{t}{2^{i} r_{B}}\right)^{N} \frac{d t}{V(x, t) t} d \mu(x)\right]^{\frac{1}{2}},
\end{aligned}
$$

where $c$ is a positive constant and $N \in \mathbf{N}$ is determined later. By (2.3), we find that, for any $x \in U_{i}(B)$,

$$
\begin{aligned}
\int_{0}^{2^{i \eta} r_{B}}\left(\frac{t}{2^{i} r_{B}}\right)^{N} \frac{d t}{V(x, t) t} & =\left(2^{i} r_{B}\right)^{-N} \sum_{j=-\infty}^{i} \int_{2^{(j-1)} r_{B}}^{2^{j \eta} r_{B}} \frac{t^{N-1} d t}{V(x, t)} \\
& \lesssim\left(2^{i} r_{B}\right)^{-N} \sum_{j=-\infty}^{i}\left(2^{j \eta} r_{B}\right)^{N}\left[V\left(x, 2^{j \eta} r_{B}\right)\right]^{-1} \\
& \lesssim 2^{-i N} \sum_{j=-\infty}^{i} 2^{j \eta N} 2^{(i-j \eta) D}\left[V\left(x_{B}, 2^{j \eta} r_{B}\right)\right]^{-1}
\end{aligned}
$$

Moreover, by (2.2), we know that, for any $j \in \mathbf{Z}$ with $j \leq i$,

$$
\left[V\left(x_{B}, 2^{j \eta} r_{B}\right)\right]^{-1} \lesssim 2^{(i-j \eta) D}\left[V\left(x_{B}, 2^{i} r_{B}\right)\right]^{-1}
$$

From this and (3.13), we deduce that, for any $i \in \mathbf{Z}_{+} \cap[11, \infty)$ and $x \in U_{i}(B)$,

$$
\begin{aligned}
\int_{0}^{2^{i \eta} r_{B}}\left(\frac{t}{2^{i} r_{B}}\right)^{N} \frac{d t}{V(x, t) t} & \lesssim 2^{-i N} \sum_{j=-\infty}^{i} 2^{j \eta N} 2^{2(i-j \eta) D}\left[V\left(x_{B}, 2^{i} r_{B}\right)\right]^{-1} \\
& \lesssim 2^{-i(N-2 D)(1-\eta)}\left[V\left(x_{B}, 2^{i} r_{B}\right)\right]^{-1}
\end{aligned}
$$

This, combined with (3.12), implies that

$$
\mathrm{I}_{1} \lesssim 2^{-\frac{i}{2}(N-2 D)(1-\eta)}\|m\|_{L^{2}(\mathcal{X})} \lesssim 2^{-\frac{i}{2}(N-2 D)(1-\eta)}[\mu(B)]^{1 / 2}\left\|\chi_{B}\right\|_{L^{p(\cdot)}(\mathcal{X})}^{-1} .
$$

From this, (3.11), (3.10), (3.9) and (3.8), it follows that, for any $(p(\cdot), M, \varepsilon)_{L^{-}}$ molecule $m$ associated with ball $B \subset \mathcal{X}$ and $i \in \mathbf{Z}_{+} \cap[11, \infty)$,

$$
\left\|S_{L}(m)\right\|_{L^{2}\left(U_{i}(B)\right)} \lesssim 2^{-i \theta}[\mu(B)]^{1 / 2}\left\|\chi_{B}\right\|_{L^{p(\cdot)}(\mathcal{X})}^{-1},
$$

where

$$
\theta:=\min \left\{\frac{1}{2}(N-2 D)(1-\eta), \varepsilon-\frac{D}{2}, 2 M \eta\right\} .
$$

By the fact that $M>\frac{D}{2}\left(\frac{1}{p_{-}}-\frac{1}{2}\right)$ and $\varepsilon \in\left(\frac{D}{p_{-}}, \infty\right)$, we choose some $\eta \in(0,1)$ and $N \in \mathbf{N}$ large enough such that $\theta \in\left(D\left(\frac{1}{p_{-}}-\frac{1}{2}\right), \infty\right)$, which, together with (3.14) and (3.7), implies that (3.5) holds true. This finishes the proof of Proposition 3.5. 
The following proposition shows that $\left[H_{L}^{p(\cdot)}(\mathcal{X}) \cap L^{2}(\mathcal{X})\right] \subset \mathbf{H}_{L, M}^{p(\cdot), \varepsilon}(\mathcal{X})$.

Proposition 3.6. Let $L$ satisfy Assumptions 2.6 and 2.7 and $p(\cdot) \in C^{\log }(\mathcal{X})$ with $p_{+} \in(0,1]$. Assume $M \in \mathbf{N}$ and $\varepsilon \in(0, \infty)$. Then, for any $f \in H_{L}^{p(\cdot)}(\mathcal{X}) \cap L^{2}(\mathcal{X})$, there exist $\left\{\lambda_{j}\right\}_{j \in \mathbf{N}} \subset \mathbf{C}$ and a family $\left\{m_{j}\right\}_{j \in \mathbf{N}}$ of $(p(\cdot), M, \varepsilon)_{L}$-molecules, associated with balls $\left\{B_{j}\right\}_{j \in \mathbf{N}}$ of $\mathcal{X}$, such that $f=\sum_{j=1}^{\infty} \lambda_{j} m_{j}$ in $L^{2}(\mathcal{X})$ and

$$
\mathcal{A}\left(\left\{\lambda_{j}\right\}_{j \in \mathbf{N}},\left\{B_{j}\right\}_{j \in \mathbf{N}}\right) \leq C\|f\|_{H_{L}^{p(\cdot)}(\mathcal{X})},
$$

where $\mathcal{A}\left(\left\{\lambda_{j}\right\}_{j \in \mathbf{N}},\left\{B_{j}\right\}_{j \in \mathbf{N}}\right)$ is as in (2.19) and the positive constant $C$ is independent of $f$.

Proof. For any $f \in H_{L}^{p(\cdot)}(\mathcal{X}) \cap L^{2}(\mathcal{X})$ and $(x, t) \in \mathcal{X}^{+}$, let $F(x, t):=t^{2} L e^{-t^{2} L}(f)(x)$. By [3, Theorem $\mathrm{F}]$, we know that $t^{2} L e^{-t^{2} L}$ is bounded from $L^{2}(\mathcal{X})$ to $T^{2}\left(\mathcal{X}^{+}\right)$. This, together with $f \in H_{L}^{p(\cdot)}(\mathcal{X}) \cap L^{2}(\mathcal{X})$, implies that $F \in T^{p(\cdot)}\left(\mathcal{X}^{+}\right) \cap T^{2}\left(\mathcal{X}^{+}\right)$. Then, by Proposition 2.13, we conclude that there exist $\left\{\lambda_{j}\right\}_{j \in \mathbf{N}} \subset \mathbf{C}$ and a family $\left\{a_{j}\right\}_{j \in \mathbf{N}}$ of $(p(\cdot), \infty)$-atoms, associated with balls $\left\{B_{j}\right\}_{j \in \mathbf{N}}$ of $\mathcal{X}$, such that

$$
F=\sum_{j=1}^{\infty} \lambda_{j} a_{j} \quad \text { in } T^{2}\left(\mathcal{X}^{+}\right) \cap T^{p(\cdot)}\left(\mathcal{X}^{+}\right)
$$

and

$$
\mathcal{A}\left(\left\{\lambda_{j}\right\}_{j \in \mathbf{N}},\left\{B_{j}\right\}_{j \in \mathbf{N}}\right) \sim\|F\|_{T^{p(\cdot)}(\mathcal{X}+)} \sim\|f\|_{H_{L}^{p(\cdot)}(\mathcal{X})} .
$$

Let $M \in \mathbf{N}$. By the $H_{\infty}$-functional calculi for $L$, we find that

$$
f=C_{(M)} \int_{0}^{\infty}\left(t^{2} L\right)^{M+1} e^{-t^{2} L}\left(t^{2} L e^{-t^{2} L}(f)\right) \frac{d t}{t}=: \pi_{M, L}(F) \quad \text { in } L^{2}\left(\mathbf{R}^{n}\right),
$$

where $C_{(M)}$ is a positive constant such that $C_{(M)} \int_{0}^{\infty} t^{2(M+2)} e^{-2 t^{2}} \frac{d t}{t}=1$ and the operator $\pi_{M, L}$ is defined by setting, for any $G \in T^{2}\left(\mathcal{X}^{+}\right)$and $x \in \mathcal{X}$,

$$
\pi_{M, L}(G)(x):=\int_{0}^{\infty}\left(t^{2} L\right)^{M+1} e^{-t^{2} L}(G(\cdot, t))(x) \frac{d t}{t} .
$$

By (2.16), Lemma 2.12 and an argument similar to that used in the proof of [60, Lemma 3.11], we conclude that $\pi_{M, L}$ is bounded from $T^{2}\left(\mathcal{X}^{+}\right)$to $L^{2}(\mathcal{X})$ and, for any $(p(\cdot), \infty)$-atom $a$ associated with ball $B \subset \mathcal{X}, \pi_{M, L}(a)$ is a harmless positive constant multiple of a $(p(\cdot), M, \varepsilon)_{L}$-molecule associated with $B$. By this, (3.17) and (3.15), we know that

$$
f=C_{(M)} \pi_{M, L}\left(\sum_{j=1}^{\infty} \lambda_{j} a_{j}\right)=C_{(M)} \sum_{j=1}^{\infty} \lambda_{j} \pi_{M, L}\left(a_{j}\right) \text { in } L^{2}(\mathcal{X}),
$$

which is a $(p(\cdot), M, \varepsilon)$-molecular representation of $f$. This, together with (3.16), then finishes the proof of Proposition 3.6.

Using Propositions 3.5 and 3.6, we can now prove Theorem 3.3.

Proof of Theorem 3.3. To prove this theorem, by a density argument, we only need to show that, if $M \in \mathbf{N} \cap\left(\frac{D}{2}\left(\frac{1}{p_{-}}-\frac{1}{2}\right), \infty\right)$ and $\varepsilon \in\left(\frac{D}{p_{-}}, \infty\right)$, then

$$
\mathbf{H}_{L, M}^{p(\cdot), \varepsilon}(\mathcal{X})=\left[H_{L}^{p(\cdot)}(\mathcal{X}) \cap L^{2}(\mathcal{X})\right] \quad \text { with equivalent quasi-norms. }
$$


Indeed, by Proposition 3.5, we find that $\mathbf{H}_{L, M}^{p(\cdot), \varepsilon}(\mathcal{X}) \subset\left[H_{L}^{p(\cdot)}(\mathcal{X}) \cap L^{2}(\mathcal{X})\right]$ and, for any $f \in \mathbf{H}_{L, M}^{p(\cdot), \varepsilon}(\mathcal{X})$,

$$
\|f\|_{H_{L}^{p(\cdot)}(\mathcal{X})} \lesssim\|f\|_{H_{L, M}^{p(\cdot), \varepsilon}(\mathcal{X})} \cdot
$$

On the other hand, from Proposition 3.6, we deduce that $\left[H_{L}^{p(\cdot)}(\mathcal{X}) \cap L^{2}(\mathcal{X})\right] \subset$ $\mathbf{H}_{L, M}^{p(\cdot), \varepsilon}(\mathcal{X})$ and, for any $f \in\left[H_{L}^{p(\cdot)}(\mathcal{X}) \cap L^{2}(\mathcal{X})\right]$,

$$
\|f\|_{H_{L, M}^{p(\cdot), \varepsilon}(\mathcal{X})} \lesssim\|f\|_{H_{L}^{p(\cdot)}(\mathcal{X})} \cdot
$$

This, together with (3.18), then finishes the proof of Theorem 3.3.

\section{Dual space of $H_{L}^{p(\cdot)}(\mathcal{X})$}

Let $L$ satisfy Assumptions 2.6 and 2.7. In this section, we show that the dual space of $H_{L}^{p(\cdot)}(\mathcal{X})$ is just the BMO-type space $\mathrm{BMO}_{p(\cdot), L^{*}}^{M}(\mathcal{X})$. Here and hereafter, we denote by $L^{*}$ the adjoint operator of $L$ on $L^{2}(\mathcal{X})$.

Let $p(\cdot) \in \mathcal{P}(\mathcal{X})$ with $p_{+} \in(0,1], L$ satisfy Assumptions 2.6 and 2.7 and $x_{0} \in \mathcal{X}$ be a fixed point. Then, for any $M \in \mathbf{N}$ and $\varepsilon \in(0, \infty)$, define

$$
\mathcal{M}_{p(\cdot), L}^{\varepsilon, M}(\mathcal{X}):=\left\{\mu:=L^{M}(\nu): \nu \in \mathcal{D}\left(L^{M}\right),\|\mu\|_{\mathcal{M}_{p(\cdot), L}^{\varepsilon, M}(\mathcal{X})}<\infty\right\},
$$

where $\mathcal{D}\left(L^{M}\right)$ denotes the domain of $L^{M}$ and

$$
\begin{aligned}
& \|\mu\|_{\mathcal{M}_{p(\cdot), L}^{\varepsilon, M}(\mathcal{X})} \\
& :=\sup _{j \in \mathbf{Z}_{+}} 2^{j \varepsilon}\left[V\left(x_{0}, 2^{j}\right)\right]^{-\frac{1}{2}}\left\|\chi_{B\left(x_{0}, 1\right)}\right\|_{L^{p(\cdot)}(\mathcal{X})} \sum_{k=0}^{M}\left\|L^{-k}(\mu)\right\|_{L^{2}\left(U_{j}\left(B\left(x_{0}, 1\right)\right)\right)} .
\end{aligned}
$$

Let

$$
\mathcal{M}_{p(\cdot), L}^{M, *}(\mathcal{X}):=\bigcap_{\varepsilon \in(0, \infty)}\left(\mathcal{M}_{p(\cdot), L}^{\varepsilon, M}(\mathcal{X})\right)^{*} .
$$

Here and hereafter, $\left(\mathcal{M}_{p(\cdot), L}^{\varepsilon, M}(\mathcal{X})\right)^{*}$ denotes the dual space of $\mathcal{M}_{p(\cdot), L}^{\varepsilon, M}(\mathcal{X})$, namely, the set of all bounded linear functionals on $\mathcal{M}_{p(\cdot), L}^{\varepsilon, M}(\mathcal{X})$ and, for any $f \in\left(\mathcal{M}_{p(\cdot), L}^{\varepsilon, M}(\mathcal{X})\right)^{*}$ and $g \in \mathcal{M}_{p(\cdot), L}^{\varepsilon, M}(\mathcal{X}),\langle f, g\rangle_{\mathcal{M}}$ denotes the duality between $\left(\mathcal{M}_{p(\cdot), L}^{\varepsilon, M}(\mathcal{X})\right)^{*}$ and $\mathcal{M}_{p(\cdot), L}^{\varepsilon, M}(\mathcal{X})$.

Remark 4.1. Let $p(\cdot) \in C^{\log }(\mathcal{X})$ with $p_{+} \in(0,1], \varepsilon \in(0, \infty)$ and $M \in \mathbf{N}$. Observe that, if $\mu \in \mathcal{M}_{p(\cdot), L}^{\varepsilon, M}(\mathcal{X})$, then $\mu$ is a harmless positive constant multiple of a $(p(\cdot), M, \varepsilon)_{L}$-molecule associated with the ball $B\left(x_{0}, 1\right)$ for $x_{0}$ as in (4.1). Conversely, if $m$ is a $(p(\cdot), M, \varepsilon)_{L}$-molecule associated with some ball $B$ of $\mathcal{X}$, then $m \in \mathcal{M}_{p(\cdot), L}^{\varepsilon, M}(\mathcal{X})$.

Definition 4.2. Let $p(\cdot) \in \mathcal{P}(\mathcal{X})$ with $p_{+} \in(0,1], M \in \mathbf{N}$ and $L$ satisfy Assumptions 2.6 and 2.7. An element $f \in \mathcal{M}_{p(\cdot), L}^{M, *}(\mathcal{X})$ is said to belong to $\mathrm{BMO}_{p(\cdot), L^{*}}^{M}(\mathcal{X})$ if

$$
\begin{aligned}
\|f\|_{\mathrm{BMO}_{p(\cdot), L^{*}}^{M}(\mathcal{X})} & :=\sup _{B \subset \mathcal{X}} \frac{[\mu(B)]^{\frac{1}{2}}}{\left\|\chi_{B}\right\|_{L^{p(\cdot)}(\mathcal{X})}}\left[\int_{B}\left|\left(I-e^{-r_{B}^{2} L^{*}}\right)^{M}(f)(x)\right|^{2} d \mu(x)\right]^{\frac{1}{2}} \\
& <\infty
\end{aligned}
$$


where the supremum is taken over all balls $B$ of $\mathcal{X}$ and $L^{*}$ denotes the adjoint operator of $L$ on $L^{2}(\mathcal{X})$.

Remark 4.3. (i) We point out that (4.2) is well defined. Indeed, for any $t \in$ $(0, \infty)$, denote either $\left(I+t^{2} L^{*}\right)^{-1}$ or $e^{-t^{2} L^{*}}$ by $A_{t, L^{*}}$. Then, for any $f \in \mathcal{M}_{p(\cdot), L}^{M, *}(\mathcal{X})$, $\left(I-A_{t, L^{*}}\right)^{M}(f) \in L_{\text {loc }}^{2}(\mathcal{X})$ in the sense of distributions (see [35, 36]). That is, for any ball $B \subset \mathcal{X}, \phi \in L^{2}(B), \varepsilon \in(0, \infty)$ and $M \in \mathbf{N},\left(I-A_{t, L}\right)^{M}(\phi) \in \mathcal{M}_{p(\cdot), L}^{\varepsilon, M}(\mathcal{X})$ and

$$
\left\langle\left(I-A_{t, L^{*}}\right)^{M}(f), \phi\right\rangle:=\left\langle f,\left(I-A_{t, L}\right)^{M}(\phi)\right\rangle_{\mathcal{M}} .
$$

(ii) An element $f \in \mathcal{M}_{p(\cdot), L^{*}}^{M, *}\left(\mathbf{R}^{n}\right)$ is said to belong to $\operatorname{BMO}_{p(\cdot), L}^{M}(\mathcal{X})$ if it satisfies (4.2) with $L^{*}$ replaced by $L$.

We have the following characterizations of the spaces $\mathrm{BMO}_{p(\cdot), L}^{M}(\mathcal{X})$.

Lemma 4.4. Let $p(\cdot) \in C^{\log }(\mathcal{X})$ with $p_{+} \in(0,1]$ and $M \in \mathbf{N}$. Then $f \in$ $\mathrm{BMO}_{p(\cdot), L}^{M}(\mathcal{X})$ if and only if $f \in \mathcal{M}_{p(\cdot), L^{*}}^{M, *}(\mathcal{X})$ and

$\|f\|_{\mathrm{BMO}_{p(\cdot), L}^{M, \text { res }(\mathcal{X})}}:=\sup _{B \subset \mathcal{X}} \frac{[\mu(B)]^{\frac{1}{2}}}{\left\|\chi_{B}\right\|_{L^{p(\cdot)}(\mathcal{X})}}\left\{\int_{B}\left|\left[I-\left(I+r_{B}^{2} L\right)^{-1}\right]^{M}(f)(x)\right|^{2} d \mu(x)\right\}^{\frac{1}{2}}<\infty$

where the supremum is taken over all balls $B$ of $\mathcal{X}$. Moreover, there exists a positive constant $C$ such that, for any $f \in \mathrm{BMO}_{p(\cdot), L}^{M}(\mathcal{X})$,

$$
C^{-1}\|f\|_{\mathrm{BMO}_{p(\cdot), L}^{M}(\mathcal{X})} \leq\|f\|_{\mathrm{BMO}_{p(\cdot), L}^{M, \text { res }}(\mathcal{X})} \leq C\|f\|_{\mathrm{BMO}_{p(\cdot), L}^{M}(\mathcal{X})} .
$$

Lemma 4.5. Let $p(\cdot) \in C^{\log }(\mathcal{X})$ with $p_{+} \in(0,1]$ and $M \in \mathbf{N}$. Then there exists a positive constant $C$ such that, for any $f \in \mathrm{BMO}_{p(\cdot), L}^{M}(\mathcal{X})$,

$$
\sup _{B \subset \mathcal{X}} \frac{[\mu(B)]^{\frac{1}{2}}}{\left\|\chi_{B}\right\|_{L^{p(\cdot)}(\mathcal{X})}}\left[\iint_{T(B)}\left|\left(t^{2} L\right)^{M} e^{-t^{2} L}(f)(x)\right|^{2} \frac{d \mu(x) d t}{t}\right]^{\frac{1}{2}} \leq C\|f\|_{\mathrm{BMO}_{p(\cdot), L}^{M}(\mathcal{X})},
$$

where the supremum is taken over all balls $B$ of $\mathcal{X}$ and $T(B)$ is as in (2.17) with $\alpha=1$.

The proofs of Lemmas 4.4 and 4.5 are similar to those of [36, Lemmas 8.1 and 8.3], respectively, the details being omitted.

In this section, we also need the following dyadic cubes due to Christ [11, Theorem 11], which shows that the metric measure space $\mathcal{X}$ has a dyadic grid analogous to that of the Euclidean space.

Lemma 4.6. [11] There exist a family of open sets, $\left\{Q_{\alpha, k}\right\}_{\alpha \in I_{k}, k \in \mathbf{Z}}$, of $\mathcal{X}$, where $I_{k}$ denotes some (possibly finite) index set depending on $k$, and constants $\delta, a_{0} \in$ $(0,1)$ and $\widetilde{C} \in(0, \infty)$ such that

(i) for any $k \in \mathbf{Z}, \mu\left(\mathcal{X} \backslash \bigcup_{\alpha \in I_{k}} Q_{\alpha, k}\right)=0$;

(ii) if $i \geq k$, then either $Q_{\alpha, i} \subset Q_{\beta, k}$ or $Q_{\alpha, i} \cap Q_{\beta, k}=\emptyset$;

(iii) for any fixed $k \in \mathbf{Z}, \alpha \in I_{k}$ and $i<k$, there exists a unique $\beta \in I_{i}$ such that $Q_{\alpha, k} \subset Q_{\beta, i}$;

(iv) for any $k \in \mathbf{Z}$ and $\alpha \in I_{k}$, the diameter of $Q_{\alpha, k}$ is not bigger than $\widetilde{C} \delta^{k}$;

(v) for any $k \in \mathbf{Z}$ and $\alpha \in I_{k}$, there exists a ball $B\left(z_{\alpha, k}, a_{0} \delta^{k}\right) \subset Q_{\alpha, k}$. 
Lemma 4.7. Let $p(\cdot) \in C^{\log }(\mathcal{X})$ with $p_{+} \in(0,1], 0<\widetilde{\varepsilon}<\varepsilon<\infty, M \in \mathbf{N}$ and $\widetilde{M}>M+\widetilde{\varepsilon}+\frac{D}{4}$. Fix $x_{0} \in \mathcal{X}$ as in (4.1). Suppose that $f \in \mathcal{M}_{p(\cdot), L}^{M, *}(\mathcal{X})$ satisfies

$$
\int_{\mathcal{X}} \frac{\left|\left[I-\left(I+L^{*}\right)^{-1}\right]^{M}(f)(x)\right|^{2}}{1+\left[d\left(x, x_{0}\right)\right]^{D+\widetilde{\varepsilon}}} d \mu(x)<\infty .
$$

Then, for any $(p(\cdot), \widetilde{M}, \varepsilon)_{L}$-molecule $m$, it holds true that

$$
\langle f, m\rangle_{\mathcal{M}}=C_{(M)} \iint_{\mathcal{X}^{+}}\left(t^{2} L^{*}\right)^{M} e^{-t^{2} L^{*}}(f)(x) \overline{t^{2} L e^{-t^{2} L}(m)(x)} \frac{d \mu(x) d t}{t},
$$

where $C_{(M)}$ is a positive constant, depending on $M$, which satisfies

$$
C_{(M)} \int_{0}^{\infty} t^{M+1} e^{2 t^{2}} \frac{d t}{t}=1 .
$$

The proof of Lemma 4.7 is analogous to that of [39, Proposition 4.6], the details being omitted. The only difference is that, instead of dealing with atoms as in [39], we work on molecules by decomposing the underline space $\mathcal{X}$ into annuli according to the ball associated with the molecule. This idea has been used in the proof of [36, Lemma 8.4].

Remark 4.8. By Lemma 4.6 and an argument similar to that used in the proof of [39, Corollary 4.3] (see also [60, Remark 4.6]), we find that, if $\widetilde{\varepsilon} \in\left(\frac{2 D}{p_{-}}, \infty\right)$, then, for any $f \in \mathrm{BMO}_{p(\cdot), L^{*}}^{M}(\mathcal{X}), f$ satisfies (4.4). Indeed, let $B:=B\left(x_{0}, 1\right)$ with $x_{0}$ as in (4.1). Then, for any $f \in \mathrm{BMO}_{p(\cdot), L^{*}}^{M}(\mathcal{X})$, we have

$$
\begin{aligned}
\mathrm{J} & :=\int_{\mathcal{X}} \frac{\left|\left[I-\left(I+L^{*}\right)^{-1}\right]^{M}(f)(x)\right|^{2}}{1+\left[d\left(x, x_{0}\right)\right]^{D+\widetilde{\varepsilon}}} d \mu(x) \\
& =\sum_{j=0}^{\infty} \int_{U_{j}(B)} \frac{\left|\left[I-\left(I+L^{*}\right)^{-1}\right]^{M}(f)(x)\right|^{2}}{1+\left[d\left(x, x_{0}\right)\right]^{D+\widetilde{\varepsilon}}} d \mu(x) \\
& \leq \sum_{j=0}^{\infty} 2^{-j(D+\widetilde{\varepsilon})} \int_{U_{j}(B)}\left|\left[I-\left(I+L^{*}\right)^{-1}\right]^{M}(f)(x)\right|^{2} d \mu(x) .
\end{aligned}
$$

In what follows, let all the notation be the same as in Lemma 4.6. For any $j \in \mathbf{Z}_{+}$, choose $k_{j} \in \mathbf{Z}$ such that $\widetilde{C} \delta^{k_{j}} \leq 2^{j}<\widetilde{C} \delta^{k_{j}-1}$ and let

$$
M_{j}:=\left\{\beta \in I_{k_{0}}: Q_{\beta, k_{0}} \cap B\left(x_{0}, \widetilde{C} \delta^{k_{j}-1}\right) \neq \emptyset\right\} .
$$

Then, by this and Lemma 4.6(i), we find that, for any $j \in \mathbf{Z}_{+}$,

$$
U_{j}(B) \subset B\left(x_{0}, \widetilde{C} \delta^{k_{j}-1}\right) \subset \bigcup_{\beta \in M_{j}} Q_{\beta, k_{0}}
$$

Moreover, by (iv) and (v) of Lemma 4.6 and the fact that $\widetilde{C} \delta^{k_{0}} \leq 1$, we know that, for any $\beta \in M_{j}$, there exists some $z_{\beta, k_{0}} \in Q_{\beta, k_{0}}$ such that

$$
B\left(z_{\beta, k_{0}}, a_{0} \delta^{k_{0}}\right) \subset Q_{\beta, k_{0}} \subset B\left(z_{\beta, k_{0}}, \widetilde{C} \delta^{k_{0}}\right) \subset B\left(z_{\beta, k_{0}}, 1\right) .
$$


From (4.5), (4.7), (4.8) and Lemma 4.4, we deduce that

$$
\begin{aligned}
\mathrm{J} & \lesssim \sum_{j=0}^{\infty} 2^{-j(D+\widetilde{\varepsilon})}\left\{\sum_{\beta \in M_{j}} \int_{B\left(z_{\beta, k_{0}}, 1\right)}\left|\left[I-\left(I+L^{*}\right)^{-1}\right]^{M}(f)(x)\right|^{2} d \mu(x)\right\} \\
& \lesssim \sum_{j=0}^{\infty} 2^{-j(D+\widetilde{\varepsilon})}\left\{\sum_{\beta \in M_{j}}\left\|\chi_{B\left(z_{\beta, k_{0}}, 1\right)}\right\|_{L^{p(\cdot)}(\mathcal{X})}^{2}\left[V\left(z_{\beta, k_{0}}, 1\right)\right]^{-1}\right\}\|f\|_{\mathrm{BMO}_{p(\cdot), L}^{M}(\mathcal{X})}^{2}
\end{aligned}
$$

By (4.6), (4.8) and Lemma 4.6(iv) with $k=k_{0}$, we conclude that

$$
B\left(z_{\beta, k_{0}}, 1\right) \subset B\left(x_{0}, 1+1+\widetilde{C} \delta^{k_{j}-1}\right) \subset B\left(x_{0}, 3 \widetilde{C} \delta^{k_{j}-1}\right) \subset B\left(x_{0}, 3 \delta^{-1} 2^{j}\right) .
$$

This, together with the fact that, for any given $r \in\left(0, p_{-}\right), \chi_{B\left(x_{0}, 3 \delta^{-1} 2^{j}\right)} \lesssim 2^{j \frac{D}{r}}\left[M\left(\chi_{B}\right)\right]^{\frac{1}{r}}$ and Lemma 2.2, implies that

$$
\left\|\chi_{B\left(z_{\beta, k_{0}}, 1\right)}\right\|_{L^{p(\cdot)}(\mathcal{X})} \lesssim 2^{j \frac{D}{r}}\left\|\left[M\left(\chi_{B}\right)\right]^{\frac{1}{r}}\right\|_{L^{p(\cdot)}(\mathcal{X})} \lesssim 2^{j \frac{D}{r}}\left\|\chi_{B}\right\|_{L^{p(\cdot)}(\mathcal{X})} \cdot
$$

On the other hand, by (2.3), we have

$$
\left[V\left(z_{\beta, k_{0}}, 1\right)\right]^{-1} \lesssim 2^{j D}\left[V\left(x_{0}, 1\right)\right]^{-1} .
$$

Since $\widetilde{\varepsilon} \in\left(\frac{2 D}{p_{-}}, \infty\right)$, it follows that there exists $r \in\left(0, p_{-}\right)$such that $\widetilde{\varepsilon} \in\left(\frac{2 D}{r}, \infty\right)$. For such an $r$, by (4.9), (4.10) and (4.11), we find that

$$
\begin{aligned}
\mathrm{J} & \lesssim \sum_{j=0}^{\infty} 2^{-j\left(\widetilde{\varepsilon}-\frac{2 D}{r}\right)}\|f\|_{\mathrm{BMO}_{p(\cdot), L}^{M}(\mathcal{X})}^{2}\left\|\chi_{B}\right\|_{L^{p(\cdot)}(\mathcal{X})}^{2}\left[V\left(x_{0}, 1\right)\right]^{-1} \\
& \sim\|f\|_{\mathrm{BMO}_{p(\cdot), L}^{M}(\mathcal{X})}^{2}\left\|\chi_{B}\right\|_{L^{p(\cdot)(\mathcal{X})}}^{2}\left[V\left(x_{0}, 1\right)\right]^{-1}<\infty .
\end{aligned}
$$

Hence, $f$ satisfies (4.4). This shows that the above claim holds true.

Theorem 4.9. Let $p(\cdot) \in C^{\log }(\mathcal{X})$ with $p_{+} \in(0,1], M \in \mathbf{N} \cap\left(\frac{D}{2}\left(\frac{1}{p_{-}}-\frac{1}{2}\right), \infty\right)$ and $\varepsilon \in\left(\frac{D}{p_{-}}, \infty\right)$. Then $\left(H_{L}^{p(\cdot)}(\mathcal{X})\right)^{*}$ coincides with $\mathrm{BMO}_{p(\cdot), L^{*}}^{M}(\mathcal{X})$ in the following sense:

(i) Let $g \in \mathrm{BMO}_{p(\cdot), L^{*}}^{M}(\mathcal{X})$. Then, for any $f \in H_{L, \text { fin, } M}^{p(\cdot), \varepsilon}(\mathcal{X})$, the linear functional $l_{g}$, given by $l_{g}(f):=\langle g, f\rangle_{\mathcal{M}}$, has a unique bounded extension to $H_{L}^{p(\cdot)}(\mathcal{X})$ and there exists a positive constant $C$ such that, for any $g \in \mathrm{BMO}_{p(\cdot), L^{*}}^{M}(\mathcal{X})$,

$$
\left\|l_{g}\right\|_{\left(H_{L}^{p(\cdot)}(\mathcal{X})\right)^{*}} \leq C\|g\|_{\mathrm{BMO}_{p(\cdot), L^{*}}^{M}(\mathcal{X})} \cdot
$$

(ii) Conversely, let $g \in\left(H_{L}^{p(\cdot)}(\mathcal{X})\right)^{*}$. Then $g \in \mathrm{BMO}_{p(\cdot), L^{*}}^{M}(\mathcal{X})$ and, for any $f \in$ $H_{L, \text { fin, } M}^{p(\cdot), \mathcal{X}}(\mathcal{X})$, it holds true that $g(f)=\langle g, f\rangle_{\mathcal{M}}$. Moreover, there exists a positive constant $C$ such that, for any $g \in\left(H_{L}^{p(\cdot)}(\mathcal{X})\right)^{*}$,

$$
\|g\|_{\mathrm{BMO}_{p(\cdot), L^{*}}^{M}(\mathcal{X})} \leq C\|g\|_{\left(H_{L}^{p(\cdot)}(\mathcal{X})\right)^{*}} .
$$

If $p(\cdot) \equiv p \in(0,1]$ is a constant exponent, Theorem 4.9 has been proved in $[24$, Theorems 3.28 and 3.29]. In particular, if $(\mathcal{X}, d, \mu):=\left(\mathbf{R}^{n},|\cdot|, d x\right)$ is the Euclidean space, Theorem 4.9 coincides with [60, Theorem 4.8].

Proof of Theorem 4.9. We first prove (i). Let $g \in \mathrm{BMO}_{p(\cdot), L^{*}}^{M}(\mathcal{X})$. By the fact that the space $H_{L, \text { fin, } M}^{p(\cdot), \mathcal{X})}$ is dense in $H_{L}^{p(\cdot)}\left(\mathbf{R}^{n}\right)$ with respect to the quasinorm $\|\cdot\|_{H_{L}^{p(\cdot)}(\mathcal{X})}$ (see Remark 3.4(iii)), to show (i), it suffices to show that, for any 
$f \in H_{L, \text { fin, } M}^{p(\cdot)}(\mathcal{X})$ with $\varepsilon \in(0, \infty)$ and $M \in \mathbf{N}$,

$$
\left|\langle g, f\rangle_{\mathcal{M}}\right| \lesssim\|g\|_{\mathrm{BMO}_{p(\cdot), L^{*}}^{M}(\mathcal{X})}\|f\|_{H_{L}^{p(\cdot)}(\mathcal{X})^{*}}
$$

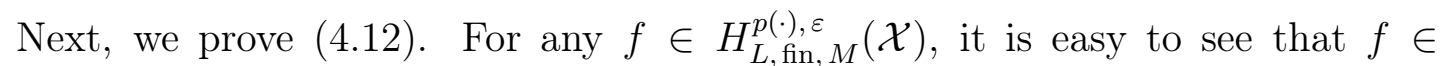
$H_{L}^{p(\cdot)}(\mathcal{X}) \cap L^{2}(\mathcal{X})$, namely, $t^{2} L e^{-t^{2} L}(f) \in T^{p(\cdot)}\left(\mathcal{X}^{+}\right) \cap T^{2}\left(\mathcal{X}^{+}\right)$. By Proposition 2.13, we know that there exist $\left\{\lambda_{j}\right\}_{j=1}^{\infty} \subset \mathbf{C}$ and a family $\left\{a_{j}\right\}_{j=1}^{\infty}$ of $(p(\cdot), \infty)$-atoms, associated with balls $\left\{B_{j}\right\}_{j \in \mathbf{N}}$ of $\mathcal{X}$, such that

$$
t^{2} L e^{-t^{2} L}(f)=\sum_{j=1}^{\infty} \lambda_{j} a_{j} \quad \text { in } T^{p(\cdot)}\left(\mathcal{X}^{+}\right) \cap T^{2}\left(\mathcal{X}^{+}\right)
$$

and

$$
\mathcal{A}\left(\left\{\lambda_{j}\right\}_{j \in \mathbf{N}},\left\{B_{j}\right\}_{j \in \mathbf{N}}\right) \sim\left\|t^{2} L e^{-t^{2} L}(f)\right\|_{T^{p(\cdot)}\left(\mathcal{X}^{+}\right)} \sim\|f\|_{H_{L}^{p(\cdot)}(\mathcal{X})} .
$$

From this, Lemma 4.7, Remark 4.8, the Hölder inequality, the fact that $\left\{a_{j}\right\}_{j \in \mathbf{N}}$ are $(p(\cdot), \infty)$-atoms, Lemma 4.5 and $(2.20)$, we deduce that, for any $f \in H_{L, \text { fin, } M}^{p(\cdot)}(\mathcal{X})$,

$$
\begin{aligned}
\left|\langle g, f\rangle_{\mathcal{M}}\right| & =\left|C_{(M)} \iint_{\mathcal{X}^{+}}\left(t^{2} L^{*}\right)^{M} e^{-t^{2} L^{*}}(g)(x) \overline{t^{2} L e^{-t^{2} L}(f)(x)} \frac{d \mu(x) d t}{t}\right| \\
& \lesssim \sum_{j=1}^{\infty}\left|\lambda_{j}\right| \iint_{\mathcal{X}^{+}}\left|\left(t^{2} L^{*}\right)^{M} e^{-t^{2} L^{*}}(g)(x)\right|\left|a_{j}(x, t)\right| \frac{d \mu(x) d t}{t} \\
& \lesssim \sum_{j=1}^{\infty}\left|\lambda_{j}\right|\left[\iint_{T\left(B_{j}\right)}\left|\left(t^{2} L^{*}\right)^{M} e^{-t^{2} L^{*}}(g)(x)\right|^{2} \frac{d \mu(x) d t}{t}\right]^{\frac{1}{2}} \\
& \times\left[\iint_{T\left(B_{j}\right)}\left|a_{j}(x, t)\right|^{2} \frac{d \mu(x) d t}{t}\right]^{\frac{1}{2}} \\
& \lesssim \sum_{j=1}^{\infty}\left|\lambda_{j}\right|\|g\|_{\mathrm{BMO}_{p(\cdot), L^{*}}^{M}(\mathcal{X})}\left\|\chi_{B_{j}}\right\|_{L^{p(\cdot)}(\mathcal{X})}\left[\mu\left(B_{j}\right)\right]^{-\frac{1}{2}}\left\|a_{j}\right\|_{T^{2}\left(\mathcal{X}^{+}\right)} \\
& \lesssim \sum_{j=1}^{\infty}\left|\lambda_{j}\right|\|g\|_{\mathrm{BMO}_{p(\cdot), L^{*}}^{M}(\mathcal{X})} \lesssim \mathcal{A}\left(\left\{\lambda_{j}\right\}_{j \in \mathbf{N}},\left\{B_{j}\right\}_{j \in \mathbf{N}}\right)\|g\|_{\mathrm{BMO}_{p(\cdot), L^{*}}^{M}(\mathcal{X})} \\
& \sim\|f\|_{H_{L}^{p(\cdot)}(\mathcal{X})}\|g\|_{\mathrm{BMO}_{p(\cdot), L^{*}}^{M}(\mathcal{X})},
\end{aligned}
$$

namely, (4.12) holds true. This finishes the proof of (i).

Next, we prove (ii). Let $g \in\left(H_{L}^{p(\cdot)}(\mathcal{X})\right)^{*}$. Then we know that, for any $f \in$ $H_{L}^{p(\cdot)}(\mathcal{X})$

$$
|g(f)| \leq\|g\|_{\left(H_{L}^{p(\cdot)}(\mathcal{X})\right)^{*}}\|f\|_{H_{L}^{p(\cdot)}(\mathcal{X})} \cdot
$$

Observing that, for any $(p(\cdot), M, \varepsilon)_{L}$-molecule $m,\|m\|_{H_{L}^{p(\cdot)}(\mathcal{X})} \lesssim 1$, we obtain

$$
|g(m)| \lesssim\|g\|_{\left(H_{L}^{p(\cdot)}(\mathcal{X})\right)^{*}}
$$

On the other hand, from Remark 4.1, it follows that, for any $h \in \mathcal{M}_{p(\cdot), L}^{\varepsilon, M}(\mathcal{X})$ with $\|h\|_{\mathcal{M}_{p(\cdot), L}^{\varepsilon, M}(\mathcal{X})}=1, h$ is a harmless positive constant multiple of a $(p(\cdot), M, \varepsilon)_{L^{-}}$ molecule associated with the ball $B\left(x_{0}, 1\right)$ for $x_{0}$ as in (4.1). Combining this and 
(4.13), we find that, for any $\varepsilon \in(0, \infty), g \in\left(\mathcal{M}_{p(\cdot), L}^{\varepsilon, M}(\mathcal{X})\right)^{*}$. Hence, $g \in \mathcal{M}_{p(\cdot), L}^{M, *}(\mathcal{X})$ and, for any $h \in \mathcal{M}_{p(\cdot), L}^{\varepsilon, M}(\mathcal{X})$,

$$
\langle g, h\rangle_{\mathcal{M}}=g(h)
$$

Next, we show that

$$
\|g\|_{\mathrm{BMO}_{p(\cdot), L^{*}}^{M}(\mathcal{X})} \lesssim\|g\|_{\left(H_{L}^{p(\cdot)}(\mathcal{X})\right)^{*}} \cdot
$$

Indeed, by Assumption 2.7, it is easy to see that, for any ball $B \subset \mathcal{X}, \varphi \in L^{2}(B)$ with $\|\varphi\|_{L^{2}(B)}=1$,

$$
\frac{[\mu(B)]^{\frac{1}{2}}}{\left\|\chi_{B}\right\|_{L^{p(\cdot)}(\mathcal{X})}}\left(I-e^{r_{B}^{2} L}\right)^{M}(\varphi)
$$

is a harmless positive constant multiple of a $(p(\cdot), M, \varepsilon)_{L}$-molecule. From this, (4.3), (4.14) and (4.13), we deduce that, for any $\varphi \in L^{2}(B)$ with $\|\varphi\|_{L^{2}(B)}=1$,

$$
\begin{aligned}
& \left|\frac{[\mu(B)]^{\frac{1}{2}}}{\left\|\chi_{B}\right\|_{L^{p(\cdot)}(\mathcal{X})}} \int_{B}\left(I-e^{-r_{B}^{2} L^{*}}\right)^{M}(g)(x) \varphi(x) d \mu(x)\right| \\
& =\left|\left\langle g, \frac{[\mu(B)]^{\frac{1}{2}}}{\left\|\chi_{B}\right\|_{L^{p(\cdot)}(\mathcal{X})}}\left(I-e^{-r_{B}^{2} L}\right)^{M}(\varphi)\right\rangle_{\mathcal{M}}\right| \lesssim\|g\|_{\left(H_{L}^{p(\cdot)}(\mathcal{X})\right)^{*}},
\end{aligned}
$$

which implies that, for any ball $B \subset \mathcal{X}$,

$$
\frac{[\mu(B)]^{\frac{1}{2}}}{\left\|\chi_{B}\right\|_{L^{p(\cdot)}(\mathcal{X})}}\left[\int_{B}\left|\left(I-e^{-r_{B}^{2} L^{*}}\right)^{M}(g)(x)\right|^{2} d \mu(x)\right]^{\frac{1}{2}} \lesssim\|g\|_{\left(H_{L}^{p(\cdot)}(\mathcal{X})\right)^{*}} .
$$

Thus, (4.15) holds true, which completes the proof of (ii) and hence the proof of Theorem 4.9 .

\section{Variable Hardy spaces associated with non-negative self-adjoint operators}

In this section, motivated by $[55,56]$, we establish the non-tangential and the radial maximal function characterizations of $H_{L}^{p(\cdot)}(\mathcal{X})$, when $L$ is a non-negative self-adjoint operator, on $L^{2}(\mathcal{X})$, having a Gaussian upper bound. Throughout this section, we always assume that $L$ is an operator satisfying the following two conditions.

Assumption 5.1. $L$ is a non-negative self-adjoint operator on $L^{2}(\mathcal{X})$.

Assumption 5.2. The kernel $W_{t}$ of $e^{-t L}$ for any $t \in(0, \infty)$ is a measurable function on $\mathcal{X} \times \mathcal{X}$ and has a Gaussian upper bound, namely, there exist positive constants $C$ and $c$ such that, for any $t \in(0, \infty)$ and $x, y \in \mathcal{X}$,

$$
\left|W_{t}(x, y)\right| \leq \frac{C}{V(x, \sqrt{t})} \exp \left(-c \frac{[d(x, y)]^{2}}{t}\right) .
$$

Remark 5.3. (i) If $L$ is a one-to-one operator satisfying Assumptions 5.1 and 5.2, then $L$ falls into the scope of operators satisfying Assumptions 2.6 and 2.7. If $L$ is an operator which only satisfies Assumptions 5.1 and 5.2, but is not one-to-one, then $L$ may not have a bounded holomorphic functional calculus on $L^{2}(\mathcal{X})$. However, even for such an operator, instead of bounded holomorphic functional calculus, we 
can use functional calculus via the spectral theorem (see (5.1) below) and we know that all the results obtained in the preceding sections still hold true.

(ii) Observe that the Gaussian upper bound for $W_{t}$ is further inherited by the time derivatives of $W_{t}$, namely, for any $k \in \mathbf{N}$, there exist positive constants $c_{(k)}$ and $C_{(k)}$ such that, for any $t \in(0, \infty)$ and almost every $x, y \in \mathcal{X}$,

$$
\left|\frac{\partial^{k}}{\partial t^{k}} W_{t}(x, y)\right| \leq \frac{C_{(k)}}{t^{k} V(x, \sqrt{t})} \exp \left(-c_{(k)} \frac{[d(x, y)]^{2}}{t}\right)
$$

(see $[35,(7.2)])$.

Recall that, in [64, Chapter XI], if $L$ is a non-negative self-adjoint operator on $L^{2}(\mathcal{X})$ and $E_{L}(\lambda)$ denotes a spectral family associated with $L$, then, for every bounded Borel function $F:[0, \infty) \rightarrow \mathbf{C}$, define the operator $F(L): L^{2}(\mathcal{X}) \rightarrow L^{2}(\mathcal{X})$ as follows:

$$
F(L):=\int_{0}^{\infty} F(\lambda) d E_{L}(\lambda)
$$

Remark 5.4. The holomorphic functional calculus defined as in (2.13) coincides with the above bounded Borel functional calculus when $L$ is a non-negative selfadjoint operator on $L^{2}(\mathcal{X})$. Indeed, if $L$ is non-negative self-adjoint on $L^{2}(\mathcal{X})$ and $\psi \in \Psi\left(S_{\mu}^{0}\right)$ with $\mu \in(0, \pi)$, then, by $(2.13)$, we know that $\psi(L)$ is defined as follows:

$$
\psi(L):=\int_{\gamma} \psi(\xi)(\xi I-L)^{-1} d \xi
$$

where $\gamma:=\left\{r e^{i \nu}: r \in(0, \infty)\right\} \cup\left\{r e^{-i \nu}: r \in(0, \infty)\right\}$, for any given $\nu \in(0, \mu)$, is a curve consisting of two rays parameterized anti-clockwise. This, together with the fact that, for any $\xi \in \mathbf{C} \backslash(0, \infty),(\xi I-L)^{-1}=\int_{0}^{\infty}(\xi-\lambda)^{-1} d E_{L}(\lambda)$ (see, for example, $[40$, p. 357]) and the Cauchy theorem, implies that

$$
\begin{aligned}
\psi(L) & =\int_{\gamma} \psi(\xi)(\xi I-L)^{-1} d \xi=\int_{\gamma} \psi(\xi) \int_{0}^{\infty}(\xi-\lambda)^{-1} d E_{L}(\lambda) d \xi \\
& =\int_{0}^{\infty} \int_{\gamma} \psi(\xi)(\xi-\lambda)^{-1} d \xi d E_{L}(\lambda)=\int_{0}^{\infty} \psi(\lambda) d E_{L}(\lambda) .
\end{aligned}
$$

Hence, (5.1) coincides with $(2.13)$ when $L$ is a non-negative self-adjoint operator on $L^{2}(\mathcal{X})$

In particular, for any $t \in(0, \infty)$, the operator $\cos (t \sqrt{L})$ is well defined on $L^{2}(\mathcal{X})$. It follows from [54, Theorem 2] and [14, Theorem 3.4] (see also [10, 58]) that the integral kernel $K_{\cos (t \sqrt{L})}$ of $\cos (t \sqrt{L})$ has the following finite speed propagation property for solutions of the corresponding wave equation:

$$
\operatorname{supp} K_{\cos (t \sqrt{L})} \subset\{(x, y) \in \mathcal{X} \times \mathcal{X}: d(x, y) \leq t\} .
$$

The following lemma is just [35, Lemma 3.5], whose proof is essentially based on (5.2). In what follows, for any $f \in \mathcal{S}(\mathbf{R})$ (the class of Schwartz functions on $\mathbf{R}$ ), its Fourier transform $\widehat{f}$ is defined by setting, for any $\xi \in \mathbf{R}, \widehat{f}(\xi):=(2 \pi)^{-1} \int_{\mathbf{R}} f(x) e^{-i x \xi} d x$. We also denote the class of all infinitely differentiable functions on $\mathbf{R}$ with compact supports by $C_{c}^{\infty}(\mathbf{R})$.

Lemma 5.5. [35] Let $\psi \in C_{c}^{\infty}(\mathbf{R})$ be an even function with $\operatorname{supp} \psi \subset(-1,1)$. Let $\Phi$ denote the Fourier transform of $\psi$. Then, for any $\kappa \in \mathbf{Z}_{+}$and $t \in(0, \infty)$, the 
integral kernel $K_{\left(t^{2} L\right)^{\kappa} \Phi(t \sqrt{L})}$ of $\left(t^{2} L\right)^{\kappa} \Phi(t \sqrt{L})$ satisfies

$$
\operatorname{supp} K_{\left(t^{2} L\right)^{\kappa} \Phi(t \sqrt{L})} \subset\{(x, y) \in \mathcal{X} \times \mathcal{X}: d(x, y) \leq t\} .
$$

Recall that, in [56], the non-tangential maximal function $f_{L}^{*}$ and the radial maximal function $f_{L}^{+}$are defined, respectively, by setting, for any $f \in L^{2}(\mathcal{X})$ and $x \in \mathcal{X}$,

$$
f_{L}^{*}(x):=\sup _{(y, t) \in \Gamma(x)}\left|e^{-t^{2} L}(f)(y)\right|
$$

where $\Gamma(x)$ is as in (1.2) with $\alpha=1$, and

$$
f_{L}^{+}(x):=\sup _{t \in(0, \infty)}\left|e^{-t^{2} L}(f)(x)\right| .
$$

Then we introduce the variable Hardy spaces associated with the non-tangential or the radial maximal function, respectively, as follows.

Definition 5.6. Let $p(\cdot) \in \mathcal{P}(\mathcal{X})$ with $p_{+} \in(0,1]$.

(i) The Hardy space $H_{L, \max }^{p(\cdot)}(\mathcal{X})$ is defined to be the completion of the space

$$
\left\{f \in L^{2}(\mathcal{X}):\left\|f_{L}^{*}\right\|_{L^{p(\cdot)}(\mathcal{X})}<\infty\right\}
$$

with respect to the quasi-norm $\|f\|_{H_{L, \max }^{p(\cdot)}(\mathcal{X})}:=\left\|f_{L}^{*}\right\|_{L^{p(\cdot)}(\mathcal{X})}$.

(ii) The Hardy space $H_{L, \text { rad }}^{p(\cdot)}(\mathcal{X})$ is defined to be the completion of the space

$$
\left\{f \in L^{2}(\mathcal{X}):\left\|f_{L}^{+}\right\|_{L^{p(\cdot)}(\mathcal{X})}<\infty\right\}
$$

with respect to the quasi-norm $\|f\|_{H_{L, \text { rad }}^{p(\cdot)}(\mathcal{X})}:=\left\|f_{L}^{+}\right\|_{L^{p(\cdot)}(\mathcal{X})}$.

The following theorem is the main result of this section, which shows that the Hardy spaces $H_{L}^{p(\cdot)}(\mathcal{X}), H_{L, \text { rad }}^{p(\cdot)}(\mathcal{X})$ and $H_{L, \text { max }}^{p(\cdot)}(\mathcal{X})$ coincide each other with equivalent quasi-norms.

Theorem 5.7. Let $p(\cdot) \in C^{\log }(\mathcal{X})$ with $p_{-} \in(0,1]$ and $L$ be an operator satisfying Assumptions 5.1 and 5.2. Then the Hardy-type spaces $H_{L}^{p(\cdot)}(\mathcal{X}), H_{L, \mathrm{rad}}^{p(\cdot)}(\mathcal{X})$ and $H_{L, \max }^{p(\cdot)}(\mathcal{X})$ coincide each other with equivalent quasi-norms.

Remark 5.8. (i) In particular, if $(\mathcal{X}, d, \mu):=\left(\mathbf{R}^{n},|\cdot|, d x\right)$ is the classical Euclidean space, Theorem 5.7 was obtained in [66].

(ii) If $p(\cdot) \equiv p \in(0,1]$ is a constant exponent, Theorem 5.7 is just [56, Theorem 1.3].

To show Theorem 5.7, we first establish the following several propositions.

Proposition 5.9. Let $p(\cdot) \in C^{\log }(\mathcal{X})$ with $p_{-} \in(0,1]$ and $L$ be an operator satisfying Assumptions 5.1 and 5.2. Then the space $H_{L, \text { rad }}^{p(\cdot)}(\mathcal{X})$ coincides $H_{L, \max }^{p(\cdot)}(\mathcal{X})$ with equivalent quasi-norms.

Proof. Let $f \in L^{2}(\mathcal{X}) \cap H_{L \text {,rad }}^{p(\cdot)}(\mathcal{X})$. Then, by (5.3) and (5.4), it is easy to see that, for any $x \in \mathcal{X}, f_{L}^{+}(x) \leq f_{L}^{*}(x)$. From this and a density argument, we deduce that

$$
H_{L, \max }^{p(\cdot)}(\mathcal{X}) \subset H_{L, \text { rad }}^{p(\cdot)}(\mathcal{X})
$$


Next, we establish the reverse inclusion. Let $N \in \mathbf{N}$. Define the maximal function $M_{N}^{* *}$ by setting, for any $f \in L^{2}(\mathcal{X})$ and $x \in \mathcal{X}$,

$$
M_{N}^{* *}(f)(x):=\sup _{y \in \mathcal{X}, t \in(0, \infty)}\left[1+\frac{d(x, y)}{t}\right]^{-N}\left|e^{-t^{2} L}(f)(y)\right| .
$$

Then, for any $f \in L^{2}(\mathcal{X})$ and $x \in \mathcal{X}$, we obtain

$$
\begin{aligned}
f_{L}^{*}(x) & =\sup _{(y, t) \in \Gamma(x)}\left|e^{-t^{2} L}(f)(y)\right| \\
& \leq \sup _{(y, t) \in \Gamma(x)}\left[1+\frac{d(x, y)}{t}\right]^{N}\left[1+\frac{d(x, y)}{t}\right]^{-N}\left|e^{-t^{2} L}(f)(y)\right| \\
& \leq 2^{N} \sup _{y \in \mathcal{X}, t \in(0, \infty)}\left[1+\frac{d(x, y)}{t}\right]^{-N}\left|e^{-t^{2} L}(f)(y)\right|=2^{N} M_{N}^{* *}(f)(x),
\end{aligned}
$$

where $\Gamma(x)$ is as in (1.2) with $\alpha=1$. On the other hand, by [56, (3.4)], we find that, if $\theta \in(0,1)$ and $N \theta>2 D$, then, for any $f \in L^{2}(\mathcal{X})$ and almost every $x \in \mathcal{X}$,

$$
M_{N}^{* *}(f)(x) \lesssim\left[M\left(\left[f_{L}^{+}\right]^{\theta}\right)(x)\right]^{1 / \theta}
$$

where $D$ is as in (2.2) and $M$ as in (2.8). Fix some $\theta \in\left(0, p_{-}\right)$and $N \in \mathbf{N}$ such that $N \theta>2 D$. From (5.7), (5.6), Lemma 2.2 and Remark 2.1(iii), it follows that, for any $f \in L^{2}(\mathcal{X})$,

$$
\begin{aligned}
\left\|f_{L}^{*}\right\|_{L^{p(\cdot)}(\mathcal{X})} & \lesssim\left\|M_{N}^{* *}(f)\right\|_{L^{p(\cdot)}(\mathcal{X})} \lesssim\left\|\left[M\left(\left[f_{L}^{+}\right]^{\theta}\right)\right]^{1 / \theta}\right\|_{L^{p(\cdot)}(\mathcal{X})} \\
& \sim\left\|M\left(\left[f_{L}^{+}\right]^{\theta}\right)\right\|_{L^{\frac{p(\cdot)}{\theta}(\mathcal{X})}}^{1 / \theta} \lesssim\left\|f_{L}^{+}\right\|_{L^{p(\cdot)}(\mathcal{X})} .
\end{aligned}
$$

This, combined with a density argument, implies that $H_{L, \text { rad }}^{p(\cdot)}(\mathcal{X}) \subset H_{L, \text { max }}^{p(\cdot)}(\mathcal{X})$. By this and (5.5), we then complete the proof of Proposition 5.9.

We now establish the atomic characterization of $H_{L}^{p(\cdot)}(\mathcal{X})$ via beginning with recalling some notions.

Definition 5.10. Let $L$ satisfy Assumptions 5.1 and $5.2, p(\cdot) \in \mathcal{P}(\mathcal{X})$ with $p_{+} \in(0,1]$ and $M \in \mathbf{N}$. A function $a \in L^{2}(\mathcal{X})$ is called a $(p(\cdot), M)_{L}$-atom, associated with $L$, if there exist a function $b \in \mathcal{D}\left(L^{M}\right)$ (the domain of $L^{M}$ ) and a ball $B:=$ $B\left(x_{B}, r_{B}\right) \subset \mathcal{X}$ with $x_{B} \in \mathcal{X}$ and $r_{B} \in(0, \infty)$ such that $a=L^{M}(b)$ and, for any $k \in\{0, \ldots, M\}$,

(i) $\operatorname{supp} L^{k}(b) \subset B$;

(ii) $\left\|\left(r_{B}^{2} L\right)^{k}(b)\right\|_{L^{2}(\mathcal{X})} \leq r_{B}^{2 M}[\mu(B)]^{1 / 2}\left\|\chi_{B}\right\|_{L^{p(\cdot)}(\mathcal{X})}^{-1}$.

Definition 5.11. Let $L$ satisfy Assumptions 5.1 and 5.2, $p(\cdot) \in \mathcal{P}(\mathcal{X})$ with $p_{+} \in(0,1]$ and $M \in \mathbf{N}$. For a measurable function $f$ on $\mathcal{X}, f=\sum_{j=1}^{\infty} \lambda_{j} a_{j}$ is called an atomic $(p(\cdot), M)$-representation of $f$ if $\left\{a_{j}\right\}_{j \in \mathbf{N}}$ is a family of $(p(\cdot), M)_{L}$-atoms, the summation converges in $L^{2}(\mathcal{X})$ and $\left\{\lambda_{j}\right\}_{j \in \mathbf{N}} \subset \mathbf{C}$ satisfies that $\mathcal{A}\left(\left\{\lambda_{j}\right\}_{j \in \mathbf{N}},\left\{B_{j}\right\}_{j \in \mathbf{N}}\right)<$ $\infty$, where, for any $j \in \mathbf{N}, B_{j}$ is the ball associated with $a_{j}$ and $\mathcal{A}\left(\left\{\lambda_{j}\right\}_{j \in \mathbf{N}},\left\{B_{j}\right\}_{j \in \mathbf{N}}\right)$ is as in (2.19). Let

$$
\mathbf{H}_{L, \text { at }, M}^{p(\cdot)}(\mathcal{X}):=\{f: f \text { has an atomic }(p(\cdot), M) \text {-representation }\} .
$$


Then the variable atomic Hardy space $H_{L, \text { at, }}^{p(\cdot)}(\mathcal{X})$ is defined to be the completion of $\mathbf{H}_{L, \text { at, } M}^{p(\cdot)}(\mathcal{X})$ with respect to the quasi-norm

$$
\begin{aligned}
\|f\|_{H_{L, \text { at }, M}^{p(\cdot)}(\mathcal{X})}:=\inf \left\{\mathcal{A}\left(\left\{\lambda_{j}\right\}_{j \in \mathbf{N}},\left\{B_{j}\right\}_{j \in \mathbf{N}}\right):\right. \\
\left.\quad f=\sum_{j=1}^{\infty} \lambda_{j} a_{j} \text { is an atomic }(p(\cdot), M) \text {-representation }\right\},
\end{aligned}
$$

where $\mathcal{A}\left(\left\{\lambda_{j}\right\}_{j \in \mathbf{N}},\left\{B_{j}\right\}_{j \in \mathbf{N}}\right)$ is as in (2.19) and the infimum is taken over all the atomic $(p(\cdot), M)$-representations of $f$ as above.

The following proposition establishes the atomic characterization of $H_{L}^{p(\cdot)}(\mathcal{X})$.

Proposition 5.12. Let $L$ satisfy Assumptions 5.1 and 5.2, and $p(\cdot) \in C^{\log }(\mathcal{X})$ with $p_{+} \in(0,1]$.

(i) Assume $M \in \mathbf{N}$. Then, for any $f \in H_{L}^{p(\cdot)}(\mathcal{X}) \cap L^{2}(\mathcal{X})$, there exist $\left\{\lambda_{j}\right\}_{j \in \mathbf{N}} \subset \mathbf{C}$ and a family $\left\{a_{j}\right\}_{j \in \mathbf{N}}$ of $(p(\cdot), M)_{L}$-atoms, associated with balls $\left\{B_{j}\right\}_{j \in \mathbf{N}}$ of $\mathcal{X}$, such that $f=\sum_{j=1}^{\infty} \lambda_{j} a_{j}$ in $L^{2}(\mathcal{X})$ and

$$
\mathcal{A}\left(\left\{\lambda_{j}\right\}_{j \in \mathbf{N}},\left\{B_{j}\right\}_{j \in \mathbf{N}}\right) \leq C\|f\|_{H_{L}^{p(\cdot)}(\mathcal{X})},
$$

where the positive constant $C$ is independent of $f$.

(ii) Assume $M \in\left(\frac{D}{2}\left(\frac{1}{p_{-}}-\frac{1}{2}\right), \infty\right)$. Then there exists a positive constant $C$ such that, for any $f \in \mathbf{H}_{L, \text { at, } M}^{p(\cdot)}(\mathcal{X})$,

$$
\|f\|_{H_{L}^{p(\cdot)}(\mathcal{X})} \leq C\|f\|_{H_{L, \text { at }, M}^{p()}(\mathcal{X})} .
$$

To prove Proposition 5.12, we need some preliminary. Take a positive function $\phi \in C_{c}^{\infty}(\mathbf{R})$ with $\operatorname{supp} \phi \in(-1,1)$. Let $\Phi$ be the Fourier transform of $\psi$. For any $M \in \mathbf{Z}_{+}$and $x \in \mathbf{R}$, let $\Psi(x):=x^{2(M+1)} \Phi(x)$ and consider the operator $\pi_{\Psi, L}$, which is defined by setting, for any $F \in T^{2}\left(\mathcal{X}^{+}\right)$and $x \in \mathcal{X}$,

$$
\pi_{\Psi, L}(F)(x):=\int_{0}^{\infty} \Psi(t \sqrt{L})(F(\cdot, t))(x) \frac{d t}{t} .
$$

The operator $\pi_{\Psi, L}$ is bounded from $T^{2}\left(\mathcal{X}^{+}\right)$to $L^{2}(\mathcal{X})$. Indeed, let $F \in T^{2}\left(\mathcal{X}^{+}\right)$. Then, by the fact that $L$ is self-adjoint, Lemma 2.12 and [3, Theorem $F$ ], we find that, for any $g \in L^{2}(\mathcal{X})$ with $\|g\|_{L^{2}(\mathcal{X})}=1$,

$$
\begin{aligned}
\left|\int_{\mathcal{X}} \pi_{\Psi, L}(F)(x) \overline{g(x)} d \mu(x)\right| & =\left|\int_{0}^{\infty} \int_{\mathcal{X}} F(x, t) \overline{\Psi(t \sqrt{L})(g)(x)} d \mu(x) \frac{d t}{t}\right| \\
& \leq\|F\|_{T^{2}\left(\mathcal{X}^{+}\right)}\|\Psi(t \sqrt{L})(g)\|_{T^{2}\left(\mathcal{X}^{+}\right)} \\
& \lesssim\|F\|_{T^{2}\left(\mathcal{X}^{+}\right)}\left[\int_{0}^{\infty}\|\Psi(t \sqrt{L})(g)\|_{L^{2}(\mathcal{X})}^{2} \frac{d t}{t}\right]^{1 / 2} \\
& \lesssim\|F\|_{T^{2}(\mathcal{X}+)}\|g\|_{L^{2}(\mathcal{X})} \lesssim\|F\|_{T^{2}\left(\mathcal{X}^{+}\right)}
\end{aligned}
$$

which further implies that $\pi_{\Psi, L}$ is bounded from $T^{2}\left(\mathcal{X}^{+}\right)$to $L^{2}(\mathcal{X})$.

The following lemma plays a key role in the proof of Proposition 5.12.

Lemma 5.13. Let $p(\cdot) \in \mathcal{P}(\mathcal{X})$ and $L$ satisfy Assumptions 5.1 and 5.2. Assume that $A$ is a $(p(\cdot), \infty)$-atom associated with a ball $B \subset \mathcal{X}$. Then, for any $M \in \mathbf{N}$, 
there exists a positive constant $C_{(M)}$, independent of $A$, such that $C_{(M)} \pi_{\Psi, L}(A)$ is a $(p(\cdot), M)_{L}$-atom associated with $2 B$.

Proof. Let $A$ be a $(p(\cdot), \infty)$-atom associated with a ball $B:=B\left(x_{B}, r_{B}\right)$ for some $x_{B} \in \mathcal{X}$ and $r_{B} \in(0, \infty)$. Define

$$
a:=\pi_{\Psi, L}(A) \quad \text { and } \quad b:=\int_{0}^{\infty} t^{2(M+1)} L \Phi(t \sqrt{L})(A(\cdot, t))(x) \frac{d t}{t},
$$

where $\pi_{\Psi, L}$ is as in (5.8). Then we obtain $a=L^{M}(b)$. Moreover, by Lemma 5.5 and the fact that $\operatorname{supp} A \subset T(B)$, we know that, for any $k \in\{0, \ldots, M\}$,

$$
\operatorname{supp} L^{k}(b) \subset 2 B \text {. }
$$

On the other hand, when $k \in\{0, \ldots, M\}$, by (5.9), (5.8) and the fact that $L$ is self-adjoint, we find that, for any $g \in L^{2}(\mathcal{X})$,

$$
\begin{aligned}
& \int_{\mathcal{X}}\left(r_{B}^{2} L\right)^{k}(b)(x) \overline{g(x)} d \mu(x) \\
& =\int_{\mathcal{X}} \int_{0}^{\infty} r_{B}^{2 k} t^{2(M+1)} L^{k+1} \Phi(t \sqrt{L})(A(\cdot, t))(x) \overline{g(x)} \frac{d t}{t} d \mu(x) \\
& =\int_{0}^{\infty} \int_{\mathcal{X}} r_{B}^{2 k} t^{2(M+1)} A(x, t) \overline{L^{k+1} \Phi(t \sqrt{L})(g)(x)} d \mu(x) \frac{d t}{t} .
\end{aligned}
$$

From this, the fact that $\operatorname{supp} A \subset T(B)$, Lemma 2.12 and (2.18), we deduce that, for any $k \in\{0, \ldots, M\}$ and $g \in L^{2}(\mathcal{X})$ with $\|g\|_{L^{2}(\mathcal{X})}=1$,

$$
\begin{aligned}
& \left|\int_{\mathcal{X}}\left(r_{B}^{2} L\right)^{k}(b)(x) \overline{g(x)} d \mu(x)\right| \\
& \leq r_{B}^{2 M} \iint_{T(B)}|A(x, t)|\left|\left(t^{2} L\right)^{k+1} \Phi(t \sqrt{L})(g)(x)\right| d \mu(x) \frac{d t}{t} \\
& \leq r_{B}^{2 M}\|A\|_{T^{2}\left(\mathcal{X}^{+}\right)}\left\|\left(t^{2} L\right)^{k+1} \Phi(t \sqrt{L})(g)\right\|_{T^{2}\left(\mathcal{X}^{+}\right)} \\
& =r_{B}^{2 M}\|A\|_{T^{2}\left(\mathcal{X}^{+}\right)}\|g\|_{L^{2}(\mathcal{X})} \lesssim r_{B}^{2 M}[\mu(B)]^{\frac{1}{2}}\left\|\chi_{B}\right\|_{L^{p(\cdot)(\mathcal{X})}}^{-1},
\end{aligned}
$$

which further implies that

$$
\left\|\left(r_{B}^{2} L\right)^{k}(b)\right\|_{L^{2}(\mathcal{X})} \lesssim r_{B}^{2 M}[\mu(B)]^{\frac{1}{2}}\left\|\chi_{B}\right\|_{L^{p(\cdot)}(\mathcal{X})}^{-1} .
$$

Combining this and (5.10), we then complete the proof of Lemma 5.13.

Using Lemma 5.13, we now show Proposition 5.12.

Proof of Proposition 5.12. We first prove (i). For any $f \in H_{L}^{p(\cdot)}(\mathcal{X}) \cap L^{2}(\mathcal{X})$ and $(x, t) \in \mathcal{X}^{+}$, let $F(x, t):=t^{2} L e^{-t^{2} L}(f)(x)$. By [3, Theorem $\left.\mathrm{F}\right]$, we know that $t^{2} L e^{-t^{2} L}$ is bounded from $L^{2}(\mathcal{X})$ to $T^{2}\left(\mathcal{X}^{+}\right)$. This, together with $f \in H_{L}^{p(\cdot)}(\mathcal{X}) \cap L^{2}(\mathcal{X})$, implies that $F \in T^{p(\cdot)}\left(\mathcal{X}^{+}\right) \cap T^{2}\left(\mathcal{X}^{+}\right)$. Then, by Proposition 2.13, we further conclude that there exist $\left\{\lambda_{j}\right\}_{j \in \mathbf{N}} \subset \mathbf{C}$ and a family $\left\{a_{j}\right\}_{j \in \mathbf{N}}$ of $(p(\cdot), \infty)$-atoms, associated with balls $\left\{B_{j}\right\}_{j \in \mathbf{N}}$ of $\mathcal{X}$, such that

$$
F=\sum_{j=1}^{\infty} \lambda_{j} a_{j} \quad \text { in } \quad T^{2}\left(\mathcal{X}^{+}\right) \cap T^{p(\cdot)}\left(\mathcal{X}^{+}\right)
$$

and

$$
\mathcal{A}\left(\left\{\lambda_{j}\right\}_{j \in \mathbf{N}},\left\{B_{j}\right\}_{j \in \mathbf{N}}\right) \sim\|F\|_{T^{p(\cdot)}\left(\mathcal{X}^{+}\right)} \sim\|f\|_{H_{L}^{p(\cdot)}(\mathcal{X})},
$$


where $\mathcal{A}\left(\left\{\lambda_{j}\right\}_{j \in \mathbf{N}},\left\{B_{j}\right\}_{j \in \mathbf{N}}\right)$ is as in (2.19).

Let $M \in \mathbf{N}$ and $\pi_{\Psi, L}$ be as in (5.8). By the bounded holomorphic functional calculi for $L$, we find that

$$
f=C_{(M)} \int_{0}^{\infty}\left(t^{2} L\right)^{M+1} \Phi(t \sqrt{L})\left(t^{2} L e^{-t^{2} L}(f)\right) \frac{d t}{t}=C_{(M)} \pi_{\Psi, L}(F) \quad \text { in } \quad L^{2}\left(\mathbf{R}^{n}\right),
$$

where $C_{(M)}$ is a positive constant such that $C_{(M)} \int_{0}^{\infty} t^{2(M+2)} \Phi(t) e^{-t^{2}} \frac{d t}{t}=1$. From the fact that $\pi_{\Psi, L}$ is bounded from $T^{2}\left(\mathcal{X}^{+}\right)$to $L^{2}(\mathcal{X}),(5.11)$ and (5.13), we deduce that

$$
f=C_{(M)} \pi_{M, L}\left(\sum_{j=1}^{\infty} \lambda_{j} a_{j}\right)=C_{(M)} \sum_{j=1}^{\infty} \lambda_{j} \pi_{M, L}\left(a_{j}\right) \text { in } L^{2}(\mathcal{X}) .
$$

Moreover, by Lemma 5.13, we find that $\left\{\pi_{M, L}\left(a_{j}\right)\right\}_{j \in \mathbf{N}}$ is a harmless positive constant multiple of a family of $(p(\cdot), M)_{L^{-}}$-atoms. Thus, (5.14) is an atomic $(p(\cdot), M)$ representation of $f$. This, together with (5.12), then finishes the proof of (i).

The proof of (ii) is similar to that of Proposition 3.5, the details being omitted. This finishes the proof of Proposition 5.12.

Proposition 5.14. Let $p(\cdot) \in C^{\log }(\mathcal{X})$ with $p_{-} \in(0,1]$ and $L$ be an operator satisfying Assumptions 5.1 and 5.2. Then there exists a positive constant $C$ such that, for any $f \in H_{L}^{p(\cdot)}(\mathcal{X})$,

$$
\|f\|_{H_{L, \max }^{p(\cdot)}(\mathcal{X})} \leq C\|f\|_{H_{L}^{p(\cdot)}(\mathcal{X})} \cdot
$$

Proof. Let $f \in H_{L}^{p(\cdot)}(\mathcal{X}) \cap L^{2}(\mathcal{X})$. By (i) of Proposition 5.12, we know that there exist $\left\{\lambda_{j}\right\}_{j \in \mathbf{N}} \subset \mathbf{C}$ and a family $\left\{a_{j}\right\}_{j \in \mathbf{N}}$ of $(p(\cdot), M)_{L}$-atoms with $M \in \mathbf{N}$, associated with balls $\left\{B_{j}\right\}_{j \in \mathbf{N}}$ of $\mathcal{X}$, such that

$$
f=\sum_{j \in \mathbf{N}} \lambda_{j} a_{j} \quad \text { in } L^{2}(\mathcal{X})
$$

and

$$
\mathcal{A}\left(\left\{\lambda_{j}\right\}_{j \in \mathbf{N}},\left\{B_{j}\right\}_{j \in \mathbf{N}}\right) \lesssim\|f\|_{H_{L}^{p(\cdot)}(\mathcal{X})} .
$$

Next, we prove that, for any $f \in H_{L}^{p(\cdot)}(\mathcal{X}) \cap L^{2}(\mathcal{X})$,

$$
\|f\|_{H_{L, \max }^{p(\cdot)}(\mathcal{X})} \lesssim \mathcal{A}\left(\left\{\lambda_{j}\right\}_{j \in \mathbf{N}},\left\{B_{j}\right\}_{j \in \mathbf{N}}\right) .
$$

To this end, we first show that the non-tangential maximal function $f_{L}^{*}$ is bounded on $L^{2}(\mathcal{X})$. Indeed, let $f \in L^{2}(\mathcal{X})$. Then, by Assumption 5.2, (2.2) and (2.3), we conclude that, for any $x \in \mathcal{X}, t \in(0, \infty)$ and $y \in B(x, t)$,

$$
\begin{aligned}
\left|e^{-t^{2} L}(f)(y)\right| & =\left|\int_{\mathcal{X}} W_{t^{2}}(y, z) f(z) d \mu(z)\right| \leq \int_{\mathcal{X}} \frac{1}{V(y, t)} e^{-c \frac{[d(y, z)]^{2}}{t^{2}}}|f(z)| d \mu(z) \\
& \leq \sum_{j=0}^{\infty} \int_{U_{j}(B(y, t))} \frac{1}{V(y, t)} e^{-4^{j}}|f(z)| d \mu(z) \\
& \lesssim \sum_{j=0}^{\infty} \frac{1}{V(x, t)} \int_{B\left(y, 2^{j} t\right)} e^{-4^{j}}|f(z)| d \mu(z)
\end{aligned}
$$




$$
\begin{aligned}
& \lesssim \sum_{j=0}^{\infty} \frac{2^{j D} e^{-4^{j}}}{V\left(x, 2^{j+1} t\right)} \int_{B\left(x, 2^{j+1} t\right)}|f(z)| d \mu(z) \\
& \lesssim \sum_{j=0}^{\infty} 2^{j D} e^{-4^{j}} M(f)(x) \lesssim M(f)(x),
\end{aligned}
$$

where $U_{j}(B(y, t))$ is as in (1.3) with $B$ replaced by $B(y, t)$. Hence, we find that, for any $f \in L^{2}(\mathcal{X})$ and $x \in \mathcal{X}, f_{L}^{*}(x) \lesssim M(f)(x)$. By this and the fact that $M$ is bounded on $L^{2}(\mathcal{X})$, we know that $f_{L}^{*}$ is bounded on $L^{2}(\mathcal{X})$. Combining this, (5.15) and the Riesz theorem, we conclude that, for any $f \in H_{L}^{p(\cdot)}(\mathcal{X}) \cap L^{2}(\mathcal{X})$ and almost every $x \in \mathcal{X}$,

$$
f_{L}^{*}(x) \leq \sum_{j=1}^{\infty}\left|\lambda_{j}\right|\left(a_{j}\right)_{L}^{*}(x) \leq \sum_{j=1}^{\infty} \sum_{k=0}^{\infty}\left|\lambda_{j}\right|\left(a_{j}\right)_{L}^{*}(x) \chi_{U_{k}\left(B_{j}\right)}(x) .
$$

Thus, we have

$$
\begin{aligned}
\left\|f_{L}^{*}\right\|_{L^{p(\cdot)}(\mathcal{X})}^{p_{-}} & \leq\left\|\left[\sum_{k=0}^{\infty} \sum_{j=1}^{\infty}\left|\lambda_{j}\right|\left(a_{j}\right)_{L}^{*} \chi_{U_{k}\left(B_{j}\right)}\right]^{p_{-}}\right\|_{\frac{L^{\frac{p(\cdot)}{p}}}{\left.\mathcal{X}^{\prime}\right)}} \\
& \leq \sum_{k=0}^{\infty}\left\|\left\{\sum_{j=1}^{\infty}\left|\lambda_{j}\right|^{p_{-}}\left[\left(a_{j}\right)_{L}^{*} \chi_{U_{k}\left(B_{j}\right)}\right]^{p_{-}}\right\}^{\frac{1}{p_{-}}}\right\|_{L^{p(\cdot)}(\mathcal{X})}^{p_{-}} .
\end{aligned}
$$

We now claim that there exist positive constants $C$ and $\theta \in\left(D\left(\frac{1}{p_{-}}-\frac{1}{2}\right), \infty\right)$ such that, for any $k \in \mathbf{Z}_{+}$and $(p(\cdot), M)_{L}$-atom $a$, associated with ball $B:=B\left(x_{B}, r_{B}\right)$ for some $x_{B} \in \mathcal{X}$ and $r_{B} \in(0, \infty)$,

$$
\left\|a_{L}^{*}\right\|_{L^{2}\left(U_{k}(B)\right)} \leq C 2^{-k \theta}[\mu(B)]^{\frac{1}{2}}\left\|\chi_{B}\right\|_{L^{p(\cdot)}(\mathcal{X})}^{-1} .
$$

If this claim is true, then, by (5.19) and an argument similar to that used in (3.6), we obtain (5.17), which, together with (5.16), then completes the proof of Proposition 5.14 .

Next, we prove the above claim (5.20). Indeed, when $k \in\{0,1,2\}$, by the boundedness of $f_{L}^{*}$ on $L^{2}(\mathcal{X})$ and Definition 5.10, we obtain

$$
\left\|a_{L}^{*}\right\|_{L^{2}\left(U_{k}(B)\right)} \lesssim\|a\|_{L^{2}(\mathcal{X})} \lesssim[\mu(B)]^{1 / 2}\left\|\chi_{B}\right\|_{L^{p(\cdot)}(\mathcal{X})}^{-1} .
$$

When $k \in \mathbf{N}$ and $k \geq 3$, for any $x \in U_{k}(B)$, we write

$$
\begin{aligned}
& a_{L}^{*}(x)=\sup _{(y, t) \in \Gamma(x)}\left|e^{-t^{2} L}(a)(y)\right| \\
& \leq \sup _{\substack{t \in\left(0, c c^{2} \eta_{\left.r_{B}\right]}\right] \\
d(x, y)<t}}\left|e^{-t^{2} L}(a)(y)\right|+\sup _{\substack{t \in\left[c 2^{\left.k \eta_{r_{B}}, \infty\right)} \\
d(x, y)<t\right.}}\left|e^{-t^{2} L}(a)(y)\right| \\
& =: \mathrm{I}(x)+\operatorname{II}(x),
\end{aligned}
$$

where $c \in(0, \infty)$ and $\eta \in(0,1)$ are constants which are determined later, and $\Gamma(x)$ is as in (1.2) with $\alpha=1$. 
We first estimate I. Observe that, for any $k \in \mathbf{N} \cap[3, \infty), x \in U_{k}(B), y \in B(x, t)$ and $z \in B$,

$$
\begin{aligned}
d(y, z) & \geq d\left(x, x_{B}\right)-d(x, y)-d\left(z, x_{B}\right) \\
& \geq 2^{k-1} r_{B}-c 2^{k \eta} r_{B}-r_{B} \geq\left(1-c 2^{3 \eta-1}\right) 2^{k-2} r_{B} .
\end{aligned}
$$

Fix $c$ small enough such that $1-c 2^{3 \eta-1} \in(0,1)$. Then, by Assumption 5.2 and (2.3), we find that, when $k \in \mathbf{N}$ and $k \geq 3$, for any $x \in U_{k}(B)$,

$$
\begin{aligned}
& \mathrm{I}(x) \leq \sup _{\substack{t \in\left(0,2 k \eta_{\left.r_{B}\right]}\right] \\
d(x, y)<t}} \int_{\mathcal{X}}\left|W_{t^{2}}(y, z) a(z)\right| d \mu(z) \\
& \lesssim \sup _{\substack{t \in\left(0,2^{k \eta}, d(x, y)<t\right.}} \int_{\mathcal{X}} \frac{1}{V(y, t)} e^{-c \frac{[d(y, z)]^{2}}{t^{2}}}|a(z)| d \mu(z) \\
& \lesssim \sup _{t \in\left(0,2^{k} \eta_{B}\right]} \int_{B\left(x_{B}, r_{B}\right)} \frac{1}{V(x, t)} e^{-c \frac{4^{k} r_{B}^{2}}{t^{2}}}|a(z)| d \mu(z) .
\end{aligned}
$$

By (2.3) and (2.2), we know that, for any $x \in U_{k}(B)$ and $t \in\left(0, c 2^{k \eta} r_{B}\right]$,

$$
\begin{aligned}
\frac{1}{V(x, t)} & \lesssim\left[1+\frac{d\left(x, x_{B}\right)}{t}\right]^{D} \frac{1}{V\left(x_{B}, t\right)} \lesssim\left[1+\frac{2^{k} r_{B}}{t}\right]^{D} \frac{1}{V\left(x_{B}, t\right)} \\
& \lesssim\left(\frac{2^{k} r_{B}}{t}\right)^{2 D} \frac{1}{V\left(x_{B}, 2^{k} r_{B}\right)} \lesssim\left(\frac{2^{k} r_{B}}{t}\right)^{2 D} \frac{1}{V\left(x_{B}, r_{B}\right)} .
\end{aligned}
$$

This, combined with (5.22) and the Hölder inequality, implies that, for any $x \in$ $U_{k}(B)$,

$$
\begin{aligned}
\mathrm{I}(x) & \lesssim \sup _{t \in\left(0, c 2^{k \eta} r_{B}\right]} e^{-c \frac{4^{k} r_{B}^{2}}{t^{2}}}\left(\frac{2^{k} r_{B}}{t}\right)^{2 D} \frac{1}{V\left(x_{B}, r_{B}\right)} \int_{B\left(x_{B}, r_{B}\right)}|a(z)| d \mu(z) \\
& \lesssim e^{-c 4^{k(1-\eta)}}[\mu(B)]^{-1 / 2}\|a\|_{L^{2}(\mathcal{X})} \lesssim 2^{-k N}\left\|\chi_{B}\right\|_{L^{p(\cdot)}(\mathcal{X})}^{-1},
\end{aligned}
$$

where $N \in \mathbf{N} \cap\left(\frac{D}{p_{-}}, \infty\right)$ is a fixed constant. Hence,

$$
\|\mathrm{I}\|_{L^{2}\left(U_{k}(B)\right)} \lesssim 2^{-N k}\left[\mu\left(2^{k} B\right)\right]^{\frac{1}{2}}\left\|\chi_{B}\right\|_{L^{p(\cdot)}(\mathcal{X})}^{-1} \lesssim 2^{-k\left(N-\frac{D}{2}\right)}[\mu(B)]^{\frac{1}{2}}\left\|\chi_{B}\right\|_{L^{p(\cdot)}(\mathcal{X})}^{-1} .
$$

Next, we estimate II. For any $x \in U_{k}(B)$, we have

$$
\begin{aligned}
\mathrm{II}(x) & =\sup _{\substack{t \in\left[c^{k} \eta_{r_{B}}, \infty\right) \\
d(x, t)<t}} r_{B}^{2 M} t^{-2 M}\left|\left(t^{2} L\right)^{M} e^{-t^{2} L}\left(r_{B}^{-2 M} b\right)(y)\right| \\
& \lesssim 2^{-2 M \eta k}\left(r_{B}^{-2 M} b\right)_{L, M}^{*}(x),
\end{aligned}
$$

where $b \in \mathcal{D}\left(L^{M}\right)$ such that $a=L^{M}(b)$ and, for any $f \in L^{2}(\mathcal{X})$ and $x \in \mathcal{X}$,

$$
f_{L, M}^{*}(x):=\sup _{(y, t) \in \Gamma(x)}\left|\left(t^{2} L\right)^{M} e^{-t^{2} L}(f)(y)\right|,
$$

where $\Gamma(x)$ is as in (1.2) with $\alpha=1$. By this, Remark 5.3(ii) and an argument similar to that used in (5.18), we find that, for any $x \in U_{k}(B)$,

$$
\mathrm{II}(x) \lesssim 2^{-2 M \eta k} M\left(r_{B}^{-2 M} b\right)(x) .
$$

Thus, we obtain

$$
\|\mathrm{II}\|_{L^{2}\left(U_{k}(B)\right)} \lesssim 2^{-2 M \eta k}[\mu(B)]^{1 / 2}\left\|\chi_{B}\right\|_{L^{p(\cdot)}(\mathcal{X})}^{-1} .
$$


By taking some $M \in\left(\frac{D}{2}\left(\frac{1}{p_{-}}-\frac{1}{2}\right), \infty\right)$ and $\eta \in(0,1)$, we have $2 M \eta>D\left(\frac{1}{p_{-}}-\frac{1}{2}\right)$. Combining this, (5.23) and (5.21), we obtain (5.20), which completes the proof of Proposition 5.14.

Let $F$ be a measurable function on $\mathcal{X}^{+}$. For any $\alpha \in(0, \infty)$ and $x \in \mathcal{X}$, define

$$
F_{\alpha}^{*}(x):=\sup _{(y, t) \in \Gamma_{\alpha}(x)}|F(y, t)|,
$$

where $\Gamma_{\alpha}(x)$ is as in (1.2).

Lemma 5.15. Let $p(\cdot) \in C^{\log }(\mathcal{X})$ with $0<p_{-} \leq p_{+}<\infty$ and $0<\alpha_{2} \leq \alpha_{1}<\infty$. If $\lambda \in\left(0, p_{-}\right)$, then there exists a positive constant $C$ such that, for any measurable function $F$ on $\mathcal{X}^{+}$,

$$
\left\|F_{\alpha_{1}}^{*}\right\|_{L^{p(\cdot)}(\mathcal{X})} \leq C\left(1+\frac{\alpha_{1}}{\alpha_{2}}\right)^{\frac{2 D}{\lambda}}\left\|F_{\alpha_{2}}^{*}\right\|_{L^{p(\cdot)}(\mathcal{X})} .
$$

Proof. Let $0<\alpha_{2} \leq \alpha_{1}<\infty$ and $\lambda \in\left(0, p_{-}\right)$be as in the assumption of this lemma. By (5.24), we know that, for any $x \in \mathcal{X}$, there exists some $(y, t) \in \Gamma_{\alpha_{1}}(x)$ such that $F_{\alpha_{1}}^{*}(x) \leq 2|F(y, t)|$. From this, we deduce that, for any $z \in B\left(y, \alpha_{2} t\right)$,

$$
F_{\alpha_{2}}^{*}(z)=\sup _{(\widetilde{z}, t) \in \Gamma_{\alpha_{2}}(z)}|F(\widetilde{z}, t)| \geq|F(y, t)| \geq \frac{1}{2} F_{\alpha_{1}}^{*}(x),
$$

which, together with the fact that $d(x, y)<\alpha_{1} t$, further implies that, for any $x \in \mathcal{X}$,

$$
\begin{aligned}
{\left[\frac{1}{2} F_{\alpha_{1}}^{*}(x)\right]^{\lambda} \leq } & \frac{1}{V\left(y, \alpha_{2} t\right)} \int_{B\left(y, \alpha_{2} t\right)}\left[F_{\alpha_{2}}^{*}(z)\right]^{\lambda} d \mu(z) \\
\leq & \frac{V\left(x, d(x, y)+\alpha_{2} t\right)}{V\left(y, \alpha_{2} t\right)} \frac{1}{V\left(x, d(x, y)+\alpha_{2} t\right)} \\
& \times \int_{B\left(x, d(x, y)+\alpha_{2} t\right)}\left[F_{\alpha_{2}}^{*}(z)\right]^{\lambda} d \mu(z) \\
\leq & \frac{V\left(x,\left(\alpha_{1}+\alpha_{2}\right) t\right)}{V\left(y, \alpha_{2} t\right)} M\left(\left[F_{\alpha_{2}}^{*}\right]^{\lambda}\right)(x) .
\end{aligned}
$$

Moreover, by (2.2) and (2.3), we have

$$
\begin{aligned}
V\left(x,\left(\alpha_{1}+\alpha_{2}\right) t\right) & \lesssim\left(1+\frac{\alpha_{1}}{\alpha_{2}}\right)^{D} V\left(x, \alpha_{2} t\right) \\
& \lesssim\left(1+\frac{\alpha_{1}}{\alpha_{2}}\right)^{D}\left[1+\frac{d(x, y)}{\alpha_{2} t}\right]^{D} V\left(y, \alpha_{2} t\right) \lesssim\left(1+\frac{\alpha_{1}}{\alpha_{2}}\right)^{2 D} V\left(y, \alpha_{2} t\right) .
\end{aligned}
$$

Combining this and (5.25), we find that, for any $x \in \mathcal{X}$,

$$
F_{\alpha_{1}}^{*}(x) \lesssim\left(1+\frac{\alpha_{1}}{\alpha_{2}}\right)^{\frac{2 D}{\lambda}}\left[M\left(\left[F_{\alpha_{2}}^{*}\right]^{\lambda}\right)(x)\right]^{\frac{1}{\lambda}} .
$$

Therefore, by the fact that $\lambda \in\left(0, p_{-}\right)$, Remark 2.1(iii) and Lemma 2.2, we obtain

$$
\begin{aligned}
\left\|F_{\alpha_{1}}^{*}\right\|_{L^{p(\cdot)}(\mathcal{X})} & \lesssim\left(1+\frac{\alpha_{1}}{\alpha_{2}}\right)^{\frac{2 D}{\lambda}}\left\|\left[M\left(\left[F_{\alpha_{2}}^{*}\right]^{\lambda}\right)(x)\right]^{\frac{1}{\lambda}}\right\|_{L^{p(\cdot)}(\mathcal{X})} \\
& \sim\left(1+\frac{\alpha_{1}}{\alpha_{2}}\right)^{\frac{2 D}{\lambda}}\left\|M\left(\left[F_{\alpha_{2}}^{*}\right]^{\lambda}\right)(x)\right\|_{L^{\frac{p(\cdot)}{\lambda}(\mathcal{X})}}^{\frac{1}{\lambda}} \lesssim\left(1+\frac{\alpha_{1}}{\alpha_{2}}\right)^{\frac{2 D}{\lambda}}\left\|F_{\alpha_{2}}^{*}\right\|_{L^{p(\cdot)}(\mathcal{X})},
\end{aligned}
$$


which completes the proof of Lemma 5.15.

Let $\varphi \in \mathcal{S}(\mathbf{R})$ be an even function. Then, for any $f \in L^{2}(\mathcal{X})$ and $x \in \mathcal{X}$, define

$$
\varphi_{L, \alpha}^{*}(f)(x):=\sup _{(y, t) \in \Gamma_{\alpha}(x)}|\varphi(t \sqrt{L})(f)(y)| .
$$

Lemma 5.16. Let $p(\cdot) \in C^{\log }(\mathcal{X})$ and $L$ satisfy Assumptions 5.1 and 5.2. Let $0<\alpha_{2} \leq \alpha_{1}<\infty$. Assume that $\varphi, \psi \in \mathcal{S}(\mathbf{R})$ are even functions satisfying $\varphi(0)=$ $\psi(0)=1$. Then there exists a positive constant $C:=C_{\left(\varphi, \psi, \alpha_{1}, \alpha_{2}\right)}$, depending on $\varphi$, $\psi, \alpha_{1}$ and $\alpha_{2}$, such that, for any $f \in L^{2}(\mathcal{X})$,

$$
\left\|\varphi_{L, \alpha_{1}}^{*}(f)\right\|_{L^{p(\cdot)}(\mathcal{X})} \leq C\left\|\psi_{L, \alpha_{2}}^{*}(f)\right\|_{L^{p(\cdot)}(\mathcal{X})} .
$$

Proof. For any $x \in \mathcal{X}$, let $\Phi(x):=\varphi(x)-\psi(x)$. To prove (5.26), by Lemma 5.15, it suffices to show that, for any $f \in L^{2}(\mathcal{X})$,

$$
\left\|\Phi_{L, 1}^{*}\right\|_{L^{p(\cdot)(\mathcal{X})}} \lesssim\left\|\psi_{L, 1}^{*}(f)\right\|_{L^{p(\cdot)}(\mathcal{X})} .
$$

Indeed, let $\lambda \in\left(\frac{2 D}{p_{-}}, \infty\right)$. Then, from the proof of [56, Proposition 2.3], we deduce that there exists a positive constant $C$, depending on $\varphi$ and $\psi$, such that, for any $f \in L^{2}(\mathcal{X})$ and $x \in \mathcal{X}$

$$
\sup _{(y, t) \in \Gamma(x)}|\Phi(t \sqrt{L})(f)(y)| \leq C \sup _{\substack{s \in(0, \infty) \\ z \in \mathcal{X}}}\left[1+\frac{d(x, z)}{s}\right]^{-\lambda}|\psi(s \sqrt{L})(f)(z)|,
$$

where $\Gamma(x)$ is as in (1.2) with $\alpha=1$. Thus, we find that, for any $f \in L^{2}(\mathcal{X})$ and $x \in \mathcal{X}$,

$$
\begin{aligned}
{\left[\Phi_{L, 1}^{*}(f)(x)\right]^{p_{-}} } & \lesssim \sup _{\substack{s \in(0, \infty) \\
z \in \mathcal{X}}}\left[1+\frac{d(x, z)}{s}\right]^{-\lambda p_{-}}|\psi(s \sqrt{L})(f)(z)|^{p_{-}} \\
& \lesssim\left[\sup _{\substack{d(x, z)<s / 2 \\
s \in(0, \infty)}}+\sum_{j=0}^{\infty} \sup _{\substack { 2^{j-1} \\
\begin{subarray}{c}{s \leq d(x, z)<2^{j} s \\
s \in(0, \infty){ 2 ^ { j - 1 } \\
\begin{subarray} { c } { s \leq d ( x , z ) < 2 ^ { j } s \\
s \in ( 0 , \infty ) } }\end{subarray}}\right]\left[1+\frac{d(x, z)}{s}\right]^{-\lambda p_{-}}|\psi(s \sqrt{L})(f)(z)|^{p_{-}} \\
& \lesssim \sum_{j=0}^{\infty} 2^{-j \lambda p_{-}}\left[\psi_{L, 2^{j}}^{*}(f)(x)\right]^{p_{-}}
\end{aligned}
$$

This, combined with Remark 2.1(iii) and Lemma 5.15, implies that

$$
\begin{aligned}
\left\|\Phi_{L, 1}^{*}(f)\right\|_{L^{p(\cdot)}(\mathcal{X})} & \left.\lesssim\left\|\left\{\sum_{j=0}^{\infty} 2^{-j \lambda p_{-}}\left[\psi_{L, 2^{j}}^{*}(f)\right]^{p_{-}}\right\}^{\frac{1}{p_{-}}}\right\| \|_{L^{p(\cdot)}(\mathcal{X})}\right\}^{\frac{1}{p_{-}}} \\
& \lesssim\left\{\sum_{j=0}^{\infty} 2^{-j \lambda p_{-}}\left\|\left[\psi_{L, 2^{j}}^{*}(f)\right]^{p_{-}}\right\|{ }_{L^{\frac{p(\cdot)}{p_{-}}(\mathcal{X})}}\right\}^{\frac{1}{p_{-}}} \\
& \sim\left\{\sum_{j=0}^{\infty} 2^{-j \lambda p_{-}}\left\|\psi_{L, 2^{j}}^{*}(f)\right\|_{L^{p(\cdot)}(\mathcal{X})}^{p_{-}}\right\}^{\frac{1}{p_{-}}} \lesssim\left\|\psi_{L, 1}^{*}(f)\right\|_{L^{p(\cdot)}(\mathcal{X})}^{p_{-}}, \\
& \lesssim\left\{\sum_{j=0}^{\infty} 2^{-j \lambda p_{-}}\left(1+2^{j}\right)^{\frac{2 D_{p_{-}}}{\eta}}\left\|\psi_{L, 1}^{*}(f)\right\|_{L^{p(\cdot)}(\mathcal{X})}^{p_{-}}\right\}^{\infty}
\end{aligned}
$$


where $\eta \in\left(0, p_{-}\right)$is a positive constant such that $\frac{2 D}{p_{-}}<\frac{2 D}{\eta}<\lambda$. By the fact that $\lambda \in\left(\frac{2 D}{p_{-}}, \infty\right)$, we know that such a constant $\eta$ always exists. Thus, we obtain (5.27) and hence (5.26), which then completes the proof of Lemma 5.16.

The following proposition shows that $H_{L, \max }^{p(\cdot)}(\mathcal{X}) \subset H_{L, \text { at }, M}^{p(\cdot)}(\mathcal{X})$.

Proposition 5.17. Let $M \in \mathbf{N}, p(\cdot) \in C^{\log }(\mathcal{X})$ and $L$ satisfy Assumptions 5.1 and 5.2. Then, for any $f \in H_{L, \max }^{p(\cdot)}(\mathcal{X}) \cap L^{2}(\mathcal{X}), f$ has an atomic $(p(\cdot), M)$-representation, namely, there exist $\left\{\lambda_{j}\right\}_{j \in \mathbf{N}} \subset \mathbf{C}$ and a sequence $\left\{a_{j}\right\}_{j \in \mathbf{N}}$ of $(p(\cdot), M)_{L}$-atoms, associated with balls $\left\{B_{j}\right\}_{j \in \mathbf{N}}$, such that $f=\sum_{j \in \mathbf{N}} \lambda_{j} a_{j}$ in $L^{2}(\mathcal{X})$ and

$$
\mathcal{A}\left(\left\{\lambda_{j}\right\}_{j \in \mathbf{N}},\left\{B_{j}\right\}_{j \in \mathbf{N}}\right) \leq C\|f\|_{H_{L, \max }^{p(\cdot)}(\mathcal{X})},
$$

where the positive constant $C$ is independent of $f$. Moreover, $H_{L, \max }^{p(\cdot)}(\mathcal{X}) \subset H_{L, \text { at, } M}^{p(\cdot)}(\mathcal{X})$ and there exists a positive constant $C$ such that, for any $f \in H_{L, \max }^{p(\cdot)}(\mathcal{X})$,

$$
\|f\|_{H_{L, \text { at }, M}^{p(\cdot)}(\mathcal{X})} \leq C\|f\|_{H_{L, \max }^{p(\cdot)}(\mathcal{X})} .
$$

Proof. To prove Proposition 5.17, we first claim that it suffices to show that, for any $f \in H_{L, \text { max }}^{p(\cdot)}(\mathcal{X}) \cap L^{2}(\mathcal{X}), f$ has an atomic $(p(\cdot), M)$-representation and (5.28) holds true. Indeed, if this claim holds true, then, from (5.28) and a density argument, we deduce that (5.29) holds true.

Now, we prove the above claim. Let $\varphi \in C_{c}^{\infty}(\mathbf{R})$ be an even function with $\operatorname{supp} \varphi \subset(-1,1)$ and $\Phi$ the Fourier transform of $\varphi$. Given any $M \in \mathbf{N}$, for any $x \in \mathbf{R}$, let $\Psi(x):=x^{2 M} \Phi(x)$. By the properties of the functional calculus of $L$ (see, for example, [64, Chapter XI]), we know that, for any $f \in L^{2}(\mathcal{X})$,

$$
f=c_{\Psi}^{-1} \int_{0}^{\infty} \Psi(t \sqrt{L}) t^{2} L e^{-r^{2} L}(f) \frac{d t}{t} \quad \text { in } L^{2}(\mathcal{X}),
$$

where $c_{\Psi}:=\int_{0}^{\infty} \Psi(t) t^{2} e^{-t^{2}} \frac{d t}{t}$. For any $x \in \mathbf{R} \backslash\{0\}$, let

$$
\eta(x):=c_{\Psi}^{-1} \int_{1}^{\infty} \Psi(t x) t^{2} x^{2} e^{-t^{2} x^{2}} \frac{d t}{t}=c_{\Psi}^{-1} \int_{x}^{\infty} \Psi(y) y e^{-y^{2}} d y
$$

and $\eta(0):=1$. Then it is easy to see that $\eta \in \mathcal{S}(\mathbf{R})$ is an even function. From (5.31), it follows that, for any given $0 \leq a \leq b<\infty$ and any $f \in L^{2}(\mathcal{X})$,

$$
\eta(a \sqrt{L})(f)-\eta(b \sqrt{L})(f)=c_{\Psi}^{-1} \int_{a}^{b} \Psi(t \sqrt{L}) t^{2} L e^{-t^{2} L}(f) \frac{d t}{t} \quad \text { in } L^{2}(\mathcal{X}) .
$$

For any $f \in L^{2}(\mathcal{X})$ and $x \in \mathcal{X}$, let

$$
M_{L}(f)(x):=\sup _{(y, t) \in \Gamma_{5}(x)}\left[\left|t^{2} L e^{-t^{2} L}(f)(y)\right|+|\eta(t \sqrt{L})(f)(y)|\right],
$$

where $\Gamma_{5}(x)$ is as in (1.2) with $\alpha=5$. Then, from Lemma 5.16, (5.27) and Lemma 5.15, we deduce that, for any $f \in H_{L, \max }^{p(\cdot)}(\mathcal{X}) \cap L^{2}(\mathcal{X})$,

$$
\left\|M_{L}(f)\right\|_{L^{p(\cdot)}(\mathcal{X})} \lesssim\|f\|_{H_{L, \max }^{p(\cdot)}(\mathcal{X})} .
$$

In what follows, for any open subset $O$ of $\mathcal{X}$, denote by $\widehat{O}$ the tent over $O$ with aperture 4, namely, $\widehat{O}:=T_{4}(O)$, where $T_{4}(O)$ is as in (2.17) with $\alpha=4$. For any $i \in \mathbf{Z}$, let

$$
O_{i}:=\left\{x \in \mathcal{X}: M_{L}(f)(x)>2^{i}\right\}
$$


Then we can decompose $\mathcal{X}^{+}$as follows:

$$
\mathcal{X}^{+}=\bigcup_{i \in \mathbf{Z}} \widehat{O_{i}}=\bigcup_{i \in \mathbf{Z}}\left(\widehat{O_{i}} \backslash \widehat{O_{i+1}}\right)=: \bigcup_{i \in \mathbf{Z}} E_{i} .
$$

Noticing that, for any $i \in \mathbf{Z}, O_{i}$ is an open subset with $\mu\left(O_{i}\right)<\infty$, by Lemma 2.14, we find that, for any $i \in \mathbf{Z}$, there exists a sequence $\left\{B_{i, k}\right\}_{k \in \mathbf{N}}:=\left\{B\left(\xi_{i, k}, r_{i, k}\right)\right\}_{k \in \mathbf{N}}$ of balls, with $\left\{\xi_{i, k}\right\}_{k \in \mathbf{N}} \subset \mathcal{X}$ and $r_{i, k}:=\operatorname{dist}\left(\xi_{i, k}, O_{i}^{\complement}\right) / 2$ for any $k \in \mathbf{N}$, such that

(i) $O_{i}=\cup_{k=1}^{\infty} B_{i, k}$;

(ii) $\left\{\frac{1}{4} B_{i, k}\right\}_{k \in \mathbf{N}}$ are pairwise disjoint.

For any measurable subset $E \subset \mathcal{X}$, let

$$
R(E):=\{(y, t) \in \mathcal{X}: \operatorname{dist}(y, E)<2 t\} .
$$

For any $i \in \mathbf{Z}$ and $k \in \mathbf{Z}_{+}$, let

$$
R\left(B_{i, 0}\right):=\emptyset \quad \text { and } \quad E_{i, k}:=E_{i} \bigcap\left[R\left(B_{i, k}\right) \backslash \bigcup_{j=0}^{k-1} R\left(B_{i, j}\right)\right] .
$$

By this and the fact that $O_{i}=\cup_{k=1}^{\infty} B_{i, k}$, we conclude that $\widehat{O_{i}} \subset\left[\cup_{j \in \mathbf{N}} R\left(B_{i, j}\right)\right]$ and $\left[E_{i_{1}, k_{1}} \cap E_{i_{2}, k_{2}}\right]=\emptyset$ if $i_{1} \neq i_{2}$ or $k_{1} \neq k_{2}$. From this, (5.33) and (5.34), we deduce that

$$
\begin{aligned}
\mathcal{X}^{+} & =\bigcup_{i \in \mathbf{Z}}\left\{E_{i} \bigcap\left[\bigcup_{k \in \mathbf{N}} R\left(B_{i, k}\right)\right]\right\} \\
& =\bigcup_{i \in \mathbf{Z}}\left[E_{i} \bigcap\left(\bigcup_{k \in \mathbf{N}}\left[R\left(B_{i, k}\right) \backslash \bigcup_{j=0}^{k-1} R\left(B_{i, j}\right)\right]\right)\right]=\bigcup_{i \in \mathbf{Z}} \bigcup_{k \in \mathbf{N}} E_{i, k} .
\end{aligned}
$$

By this and (5.30), we know that, for any $f \in H_{L, \max }^{p(\cdot)}(\mathcal{X}) \cap L^{2}(\mathcal{X})$,

$$
f=\sum_{i \in \mathbf{Z}} \sum_{k \in \mathbf{N}} c_{\Psi}^{-1} \int_{0}^{\infty} \Psi(t \sqrt{L})\left(\chi_{E_{i, k}} t^{2} L e^{-t^{2} L}(f)\right) \frac{d t}{t} \quad \text { in } L^{2}(\mathcal{X})
$$

(see [56, p. 11] for more details on this fact). For any $i \in \mathbf{Z}$ and $k \in \mathbf{N}$, let

$$
\lambda_{i, k}:=2^{i}\left\|\chi_{B_{i, k}}\right\|_{L^{p(\cdot)(\mathcal{X})}}, \quad b_{i, k}:=c_{\Psi}^{-1} \lambda_{i, k}^{-1} \int_{0}^{\infty} t^{2 M} \Phi(t \sqrt{L})\left(\chi_{E_{i, k}} t^{2} L e^{-t^{2} L}(f)\right) \frac{d t}{t}
$$

and

$$
a_{i, k}:=L^{M}\left(b_{i, k}\right)=c_{\Psi}^{-1} \lambda_{i, k}^{-1} \int_{0}^{\infty} \Psi(t \sqrt{L})\left(\chi_{E_{i, k}} t^{2} L e^{-t^{2} L}(f)\right) \frac{d t}{t} .
$$

Then we have $f=\sum_{i \in \mathbf{Z}} \sum_{k \in \mathbf{N}} \lambda_{i, k} a_{i, k}$ in $L^{2}(\mathcal{X})$. Moreover, by [56, p. 12], we know that, when $i \in \mathbf{Z}$ and $k \in \mathbf{N}$, for any $j \in\{0, \ldots, M\}$,

$$
\operatorname{supp} L^{j}\left(b_{i, k}\right) \subset B\left(\xi_{i, k}, 8 r_{i, k}\right)
$$

and there exists a positive constant $C:=C_{(\Psi)}$, depending on $\Psi$, such that

$$
\left\|\left(r_{i, k}^{2} L\right)^{j}\left(b_{i, k}\right)\right\|_{L^{\infty}(\mathcal{X})} \leq C r_{i, k}^{2 M}\left\|\chi_{B_{i, k}}\right\|_{L^{p(\cdot)}(\mathcal{X})}^{-1} .
$$

This, together with (5.35), implies that $\left\{a_{i, k}\right\}_{i \in \mathbf{Z}, k \in \mathbf{N}}$ is a harmless positive constant multiple of a family of $(p(\cdot), M)_{L}$-atoms associated with balls $\left\{8 B_{i, k}\right\}_{i \in \mathbf{Z}, k \in \mathbf{N}}$. Therefore, to complete the proof of this proposition, it remains to show that

$$
\mathcal{A}\left(\left\{\lambda_{i, k}\right\}_{i \in \mathbf{Z}, k \in \mathbf{N}},\left\{8 B_{i, k}\right\}_{i \in \mathbf{Z}, k \in \mathbf{N}}\right) \lesssim\|f\|_{H_{L, \max }^{p(\cdot)}(\mathcal{X})} .
$$


Indeed, we have

$$
\begin{aligned}
& \mathcal{A}\left(\left\{\lambda_{i, k}\right\}_{i \in \mathbf{Z}, k \in \mathbf{N}},\left\{8 B_{i, k}\right\}_{i \in \mathbf{Z}, k \in \mathbf{N}}\right) \\
& =\left\|\left\{\sum_{i \in \mathbf{Z}} \sum_{k \in \mathbf{N}}\left[\frac{\left|\lambda_{i, k}\right| \chi_{8 B_{i, k}}}{\left\|\chi_{8 B_{i, k}}\right\|_{L^{p(\cdot)}(\mathcal{X})}}\right]^{p_{-}}\right\}^{\frac{1}{p_{-}}}\right\|_{L^{p(\cdot)}(\mathcal{X})}=\left\|\left\{\sum_{i \in \mathbf{Z}} \sum_{k \in \mathbf{N}} 2^{i p_{-}} \chi_{8 B_{i, k}}\right\}^{\frac{1}{p_{-}}}\right\|_{L^{p(\cdot)}(\mathcal{X})} .
\end{aligned}
$$

By this, the fact that, for any ball $B$ of $\mathcal{X}, \chi_{8 B} \lesssim M\left(\chi_{\frac{1}{4} B}\right)$, Remark 2.1(iii), Lemma 2.3 and the above properties (i) and (ii) of $B_{i, k}$, we conclude that, for any given $r \in\left(0, p_{-}\right)$,

$$
\begin{aligned}
& \mathcal{A}\left(\left\{\lambda_{i, k}\right\}_{i \in \mathbf{Z}, k \in \mathbf{N}},\left\{8 B_{i, k}\right\}_{i \in \mathbf{Z}, k \in \mathbf{N})}\right. \\
& \lesssim\left\|\left\{\sum_{i \in \mathbf{Z}} \sum_{k \in \mathbf{N}} 2^{i p_{-}}\left[M\left(\chi_{\frac{1}{4} B_{i, k}}\right)\right]^{\frac{p_{-}}{r}}\right\}^{\frac{r}{p_{-}}}\right\|_{L^{\frac{1}{r}(\cdot)}}^{\frac{1}{r}}(\mathcal{X}) \\
& \lesssim\left\|\left\{\sum_{i \in \mathbf{Z}} \sum_{k \in \mathbf{N}}\left[2^{i r} \chi_{\frac{1}{4} B_{i, k}}\right]^{\frac{p_{-}}{r}}\right\}^{\frac{1}{p_{-}}}\right\|_{L^{p(\cdot)}(\mathcal{X})} \lesssim\left\|\left\{\sum_{i \in \mathbf{Z}} 2^{i p_{-}} \chi_{O_{i}}\right\}^{\frac{1}{p_{-}}}\right\|_{L^{p(\cdot)}(\mathcal{X})} \\
& \sim\left\|\left\{\sum_{i \in \mathbf{Z}} 2^{i p_{-}} \chi_{O_{i} \backslash O_{i+1}}\right\}^{\frac{1}{p_{-}}}\right\| M_{L}(f) \|_{L^{p(\cdot)}(\mathcal{X})} \cdot
\end{aligned}
$$

This, combined with (5.32), then finishes the proof of Proposition 5.17.

Finally, using the above several propositions, we show Theorem 5.7.

Proof of Theorem 5.7. Let $p(\cdot) \in C^{\log }(\mathcal{X})$ with $p_{-} \in(0,1]$ and $L$ be an operator satisfying Assumptions 5.1 and 5.2. Then, from Proposition 5.9, we deduce that

$$
H_{L, \text { rad }}^{p(\cdot)}(\mathcal{X})=H_{L, \text { max }}^{p(\cdot)}(\mathcal{X}) \quad \text { with equivalent quasi-norms. }
$$

Let $M \in \mathbf{N} \cap\left(\frac{D}{2}\left(\frac{1}{p_{-}}-\frac{1}{2}\right), \infty\right)$. Then, by Proposition 5.12 and a density argument, we find that the space $H_{L}^{p(\cdot)}(\mathcal{X})$ coincides the space $H_{L, \text { at }, M}^{p(\cdot)}(\mathcal{X})$ with equivalent quasi-norms. Combining this and (5.29), we conclude that, for any $f \in H_{L, \text { max }}^{p(\cdot)}(\mathcal{X})$, $\|f\|_{H_{L}^{p(\cdot)}(\mathcal{X})} \lesssim\|f\|_{H_{L, \max }^{p(\cdot)}(\mathcal{X})}$. This, together with Proposition 5.14, implies that

$$
H_{L}^{p(\cdot)}(\mathcal{X})=H_{L, \max }^{p(\cdot)}(\mathcal{X}) \quad \text { with equivalent quasi-norms. }
$$

By this and (5.36), we then complete the proof of Theorem 5.7.

Acknowledgements. Junqiang Zhang would like to thank Professor Ciqiang Zhuo for some helpful conversations on this topic.

\section{References}

[1] Acerbi, E., and G. Mingione: Gradient estimates for the $p(x)$-Laplacean system. - J. Reine Angew. Math. 584, 2005, 117-148.

[2] Adamowicz, T., P. Harjulehto, and P. Hëstö: Maximal operator in variable exponent Lebesgue spaces on unbounded quasimetric measure spaces. - Math. Scand. 116, 2015, 5-22.

[3] Albrecht, D., X. T. Duong, and A. McIntosh: Operator theory and harmonic analysis In: Instructional Workshop on Analysis and Geometry, Part III (Canberra, 1995), Proc. Centre Math. Appl. Austral. Nat. Univ. 34, 1996, 77-136. 
[4] Amenta, A.: Tent spaces over metric measure spaces under doubling and related assumptions. - In: Operator theory in harmonic and non-commutative analysis, Oper. Theory Adv. Appl. 240, 2014, 1-29.

[5] Auscher, P., X. T. Duong, and A. McIntosh: Boundedness of Banach space valued singular integral operators and Hardy spaces. - Unpublished manuscript, 2005.

[6] Auscher, P., S. Hofmann, M. Lacey, A. McIntosh, and Ph. Tchamitchian: The solution of the Kato square root problem for second order elliptic operators on $\mathbf{R}^{n}$. - Ann. of Math. (2) 156, 2002, 633-654.

[7] Auscher, P., and J. M. Martell: Weighted norm inequalities, off-diagonal estimates and elliptic operators. II. Off-diagonal estimates on spaces of homogeneous type. - J. Evol. Equ. 7, $2007,265-316$.

[8] Bui, T. A., and J. Li: Orlicz-Hardy spaces associated to operators satisfying bounded $H^{\infty}$ functional calculus and Davies-Gaffney estimates. - J. Math. Anal. Appl. 373, 2011, 485-501.

[9] Calderón,.A. P.: An atomic decomposition of distributions in parabolic $H^{p}$ spaces, - Adv. Math. 25, 1977, 216-225.

[10] Cheeger, J., M. Gromov, and M. Taylor: Finite propagation speed, kernel estimates for functions of the Laplace operator, and the geometry of complete Riemannian manifolds. - J. Differential Geom. 17, 1982, 15-53.

[11] Christ, M.: A $T(b)$ theorem with remarks on analytic capacity and the Cauchy integral. Colloq. Math. 60/61, 1990, 601-628.

[12] Coifman, R. R., and G. Weiss: Analyse harmonique non-commutative sur certains espaces homogènes, étude de certaines intégrales singulières. - Lecture Notes in Math. 242, SpringerVerlag, Berlin-New York, 1971 (in French).

[13] Coifman, R. R., and G. Weiss: Extensions of Hardy spaces and their use in analysis. - Bull. Amer. Math. Soc. 83, 1977, 569-645.

[14] Coulhon, T., and A. Sikora: Gaussian heat kernel upper bounds via the Phragmén-Lindelöf theorem. - Proc. Lond. Math. Soc. (3) 96, 2008, 507-544.

[15] Cowling, M., I. Doust, A. McIntosh, and A. Yagi: Banach space operators with a bounded $H^{\infty}$ functional calculus. - J. Austral. Math. Soc. Ser. A 60, 1996, 51-89.

[16] Cruz-Uribe, D.: The Hardy-Littlewood maximal operator on variable- $L^{p}$ spaces. - In: Seminar of Mathematical Analysis (Malaga/Seville, 2002/2003), Colecc. Abierta 64, 2003, 147-156.

[17] Cruz-Uribe, D. V., and A. Fiorenza: Variable Lebesgue spaces, foundations and harmonic analysis. - Applied and Numerical Harmonic Analysis, Birkhäuser/Springer, Heidelberg, 2013.

[18] Cruz-Uribe, D., A. Fiorenza, J. M. Martell, and C. Pérez: The boundedness of classical operators on variable $L^{p}$ spaces. - Ann. Acad. Sci. Fenn. Math. 31, 2006, 239-264.

[19] Cruz-Uribe, D., and L.-A. D. Wang: Variable Hardy spaces. - Indiana Univ. Math. J. 63, $2014,447-493$.

[20] Davies, E. B.: Heat kernels and spectral theory. - Cambridge Tracts in Math. 92, Cambridge Univ. Press, Cambridge, 1989.

[21] Davies, E. B.: Uniformly elliptic operators with measurable coefficients. - J. Funct. Anal. 132, 1995, 141-169.

[22] Diening, L.: Maximal function on generalized Lebesgue spaces $L^{p(\cdot)}$. - Math. Inequal. Appl. $7,2004,245-253$.

[23] Diening, L., P. Harjulehto, P. Hästö, and M. RůŽıČKa: Lebesgue and Sobolev spaces with variable exponents. - Lecture Notes in Math. 2017, Springer, Heidelberg, 2011.

[24] Duong, X. T., and J. Li: Hardy spaces associated to operators satisfying Davies-Gaffney estimates and bounded holomorphic functional calculus. - J. Funct. Anal. 264, 2013, 14091437. 
[25] Duong, X. T., and L. YAn: New function spaces of BMO type, the John-Nirenberg inequality, interpolation, and applications. - Comm. Pure Appl. Math. 58, 2005, 1375-1420.

[26] Duong, X. T., and L. YAn: Duality of Hardy and BMO spaces associated with operators with heat kernel bounds. - J. Amer. Math. Soc. 18, 2005, 943-973.

[27] Fefferman, C., and E. M. Stein: $H^{p}$ spaces of several variables. - Acta Math. 129, 1972, $137-193$.

[28] Gaffney, M.: The conservation property of the heat equation on Riemannian manifolds. Comm. Pure Appl. Math. 12, 1959, 1-11.

[29] Gorosito, O., G. Pradolini, and O. Salinas: Boundedness of fractional operators in weighted variable exponent spaces with non doubling measures. - Czechoslovak Math. J. 60:135, 2010, 1007-1023.

[30] Grafakos, L., L. LiU, and D. Yang: Vector-valued singular integrals and maximal functions on spaces of homogeneous type. - Math. Scand. 104, 2009, 296-310.

[31] HaAse, M.: The functional calculus for sectorial operators. - Operator Theory: Advances and Applications 169, Birkhäuser Verlag, Basel, 2006.

[32] Hajibayov, M., and S. Samko: Generalized potentials in variable exponent Lebesgue spaces on homogeneous spaces. - Math. Nachr. 284, 2011, 53-66.

[33] Harjulehto, P., P. Hästö, and V. Latvala: Minimizers of the variable exponent, nonuniformly convex Dirichlet energy. - J. Math. Pures Appl. (9) 89, 2008, 174-197.

[34] Harjulehto, P., P. Hästö, and M. Pere: Variable exponent Lebesgue spaces on metric spaces: the Hardy-Littlewood maximal operator. - Real Anal. Exchange 30, 2004/05, 87-103.

[35] Hofmann, S., G. Lu, D. Mitrea, M. Mitrea, and L. Yan: Hardy spaces associated to non-negative self-adjoint operators satisfying Davies-Gaffney estimates. - Mem. Amer. Math. Soc. 214:1007, 2011.

[36] Hofmann, S., and S. Mayboroda: Hardy and BMO spaces associated to divergence form elliptic operators. - Math. Ann. 344, 2009, 37-116.

[37] Hofmann, S., S. Mayboroda, and A. McIntosh: Second order elliptic operators with complex bounded measurable coefficients in $L^{p}$, Sobolev and Hardy spaces. - Ann. Sci. Éc. Norm. Supér. (4) 44, 2011, 723-800.

[38] JiAnG, R., and D. YANG: New Orlicz-Hardy spaces associated with divergence form elliptic operators. - J. Funct. Anal. 258, 2010, 1167-1224.

[39] JIANG, R., and D. YANG: Orlicz-Hardy spaces associated with operators satisfying DaviesGaffney estimates. - Commun. Contemp. Math. 13, 2011, 331-373.

[40] Kato, T.: Perturbation theory for linear operators. - Reprint of the 1980 edition, Classics in Mathematics, Springer-Verlag, Berlin, 1995.

[41] Kokilashvili, V., and S. Samko: The maximal operator in weighted variable spaces on metric measure spaces. - Proc. A. Razmadze Math. Inst. 144, 2007, 137-144.

[42] KováčIK, O., and J. RÁKosník: On spaces $L^{p(x)}$ and $W^{k, p(x)}$. - Czechoslovak Math. J. 41:116, $1991,592-618$.

[43] Macías, R. A.: and C. Segovia: A decomposition into atoms of distributions on spaces of homogeneous type. - Adv. Math. 33, 1979, 271-309.

[44] McIntosh, A.: Operators which have an $H^{\infty}$ functional calculus. - In: Miniconference on Operator Theory and Partial Differential Equations (North Ryde, 1986), Proc. Centre Math. Anal. Austral. Nat. Univ. 14, 1986, 210-231.

[45] NAKAI, E., and Y. SAWANO: Hardy spaces with variable exponents and generalized Campanato spaces. - J. Funct. Anal. 262, 2012, 3665-3748.

[46] NakaI, E., and K. Yabuta: Pointwise multipliers for functions of weighted bounded mean oscillation on spaces of homogeneous type. - Math. Japon. 46, 1997, 15-28. 
[47] Nakano, H.: Modulared semi-ordered linear spaces. - Maruzen Co., Ltd., Tokyo, 1950.

[48] Nakano, H.: Topology of linear topological spaces. - Maruzen Co., Ltd., Tokyo, 1951.

[49] Orlicz, W.: Über konjugierte Exponentenfolgen. - Studia Math. 3, 1931, 200-211.

[50] Ouhabaz, E. M.: Analysis of heat equations on domains. - London Math. Soc. Monogr. Ser. 31, Princeton Univ. Press, Princeton, NJ, 2005.

[51] Russ, E.: The atomic decomposition for tent spaces on spaces of homogeneous type. - In: CMA/AMSI Research Symposium "Asymptotic Geometric Analysis, Harmonic Analysis, and Related Topics", - Proc. Centre Math. Appl. Austral. Nat. Univ. 42, 2007, 125-135.

[52] Sanchón, M., and J. Urbano: Entropy solutions for the $p(x)$-Laplace equation. - Trans. Amer. Math. Soc. 361, 2009, 6387-6405.

[53] Sawano, Y.: Atomic decompositions of Hardy spaces with variable exponents and its application to bounded linear operators. - Integral Equations Operator Theory 77, 2013, 123-148.

[54] Sikora, A.: Riesz transform, Gaussian bounds and the method of wave equation. - Math. Z. 247, 2004, 643-662.

[55] Song, L., and L. YAN: A maximal function characterization for Hardy spaces associated to nonnegative self-adjoint operators satisfying Gaussian estimates. - Adv. Math. 287, 2016, 463-484.

[56] Song, L., and L. YAn: Maximal function characterizations for Hardy spaces associated to nonnegative self-adjoint operators on spaces of homogeneous type. - arXiv:1605.07701.

[57] Stein, E. M., and G. Weiss: On the theory of harmonic functions of several variables. I. The theory of $H^{p}$-spaces. - Acta Math. 103, 1960, 25-62.

[58] TAYLOR, M. E.: $L^{p}$-estimates on functions of the Laplace operator. - Duke Math. J. 58, 1989, $773-793$.

[59] YANG, D., and S. YANG: Musielak-Orlicz-Hardy spaces associated with operators and their applications. - J. Geom. Anal. 24, 2014, 495-570.

[60] YAng, D., J. Zhang, and C. Zhuo: Variable Hardy spaces associated with operators satisfying Davies-Gaffney estimates. - Proc. Edinb. Math. Soc. (2) (to appear).

[61] YANG, D., and C. ZHUO: Molecular characterizations and dualities of variable exponent Hardy spaces associated with operators. - Ann. Acad. Sci. Fenn. Math. 41, 2016, 357-398.

[62] YAng, D., C. Zhuo, and E. NAKAI: Characterizations of variable exponent Hardy spaces via Riesz transforms. - Rev. Mat. Complut. 29, 2016, 245-270.

[63] YANG, S., and D. YANG: Maximal function characterizations of Musielak-Orlicz-Hardy spaces associated to non-negative self-adjoint operators satisfying Gaussian estimates. - Commun. Pure Appl. Anal. 15, 2016, 2135-2160.

[64] Yosida, K.: Functional analysis. Sixth edition. - Grundlehren Math. Wiss. 123, SpringerVerlag, Berlin-New York, 1980.

[65] Zhuo, C., Y. Sawano, and D. Yang: Hardy spaces with variable exponents on RD-spaces and applications. - Dissertationes Math. (Rozprawy Mat.) 520, 2016, 1-74.

[66] Zhuo, C., and D. YANG: Maximal function characterizations of variable Hardy spaces associated with non-negative self-adjoint operators satisfying Gaussian estimates. - Nonlinear Anal. $141,2016,16-42$.

[67] Zhuo, C., D. Yang, and Y. Liang: Intrinsic square function characterizations of Hardy spaces with variable exponents. - Bull. Malays. Math. Sci. Soc. 39, 2016, 1541-1577. 